\title{
Photoluminescent, Upconversion Luminescent and Nonlinear Optical Metal-organic Frameworks: From Fundamental Photophysics to Potential Applications
}

Tu N. Nguyen,* Fatmah Mish Ebrahim, Kyriakos Stylianou*

Laboratory of Molecular Simulation, Institut des sciences et ingénierie chimiques (ISIC), Ecole polytechnique fédérale de Lausanne (EPFL Valais), Rue de l'Industrie 17, 1951 Sion, Switzerland.

Keywords: metal-organic framework, luminescence, white-light emission, bioimaging, sensing, nonlinear optics, second harmonic generation, two-photon absorption, upconversion. Dedicated to Professor George Christou on the occasion of his 65th birthday.

*Corresponding authors: ngoctu.nguyen@epfl.ch and kyriakos.stylianou@epfl.ch

\begin{abstract}
Metal-organic frameworks (MOFs) are a class of porous materials prepared by the selfassembly of metal ions or clusters with organic ligands. The unique characteristics of MOFs, including structural tunability, high surface areas, low densities and tailored pore surface functionalization, have made them leading contenders as high-performance porous materials, alongside the established zeolites and activated carbons. Consequently, the permanent porosity of MOFs has been extensively exploited for gas capture and separation and catalysis. In recent years, the field has been expanded towards new applications and many studies of MOFs are venturing into unexplored avenues. A large number of studies have been focused on photoluminescent, upconversion luminescent, and nonlinear optical MOFs for applications in areas such as white-light emission, bioimaging, sensing, and photocatalysis. Within the first half of this tutorial review, we present the fundamental principles of luminescence, including detailed scientific discussions on the luminescence origin of different materials such as organic dyes, transition metal complexes, quantum dots, and lanthanide compounds.
\end{abstract}


Principles and important parameters for the applications of luminescent MOFs are introduced, followed by a summary of recent interesting publications for each application. In the second half, we introduce nonlinear optical effects including second harmonic generation and two-photon absorption, and upconversion of luminescence, followed by detailed examples of MOFs that exhibit these phenomena. Finally, insights about the remaining challenges and future directions are discussed. 


\section{Table of Contents}

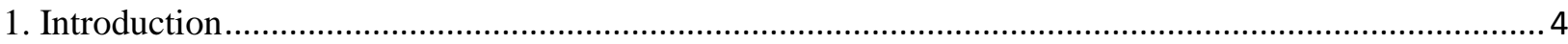

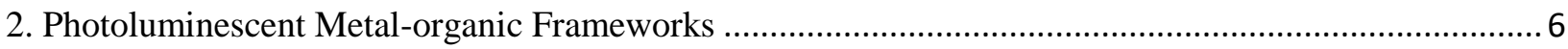

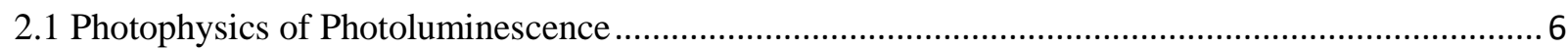

2.2 Applications of Luminescent Metal-Organic Frameworks ......................................................... 18

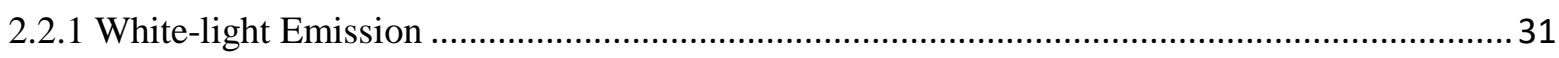

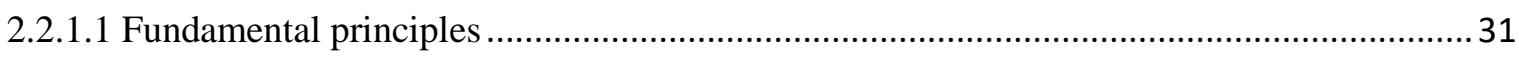

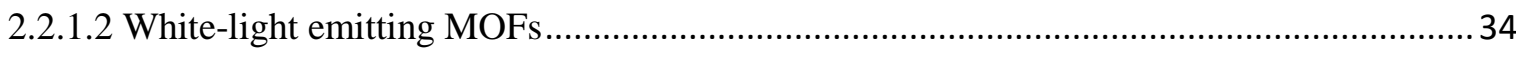

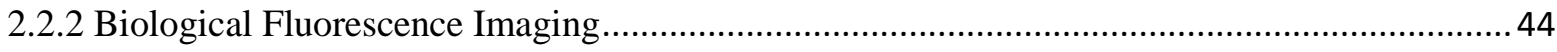

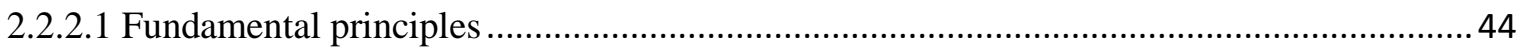

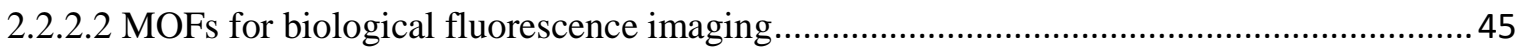

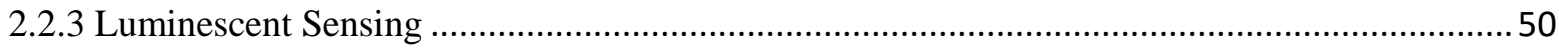

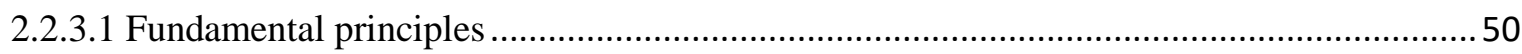

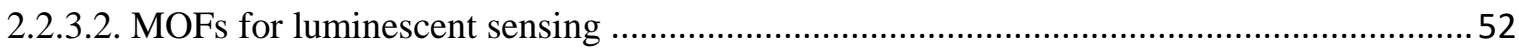

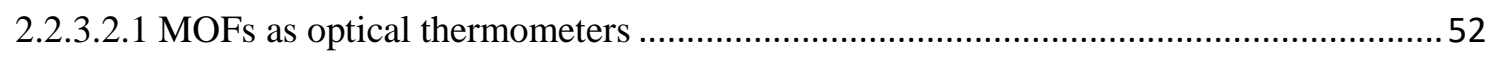

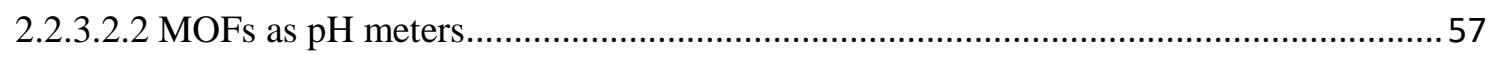

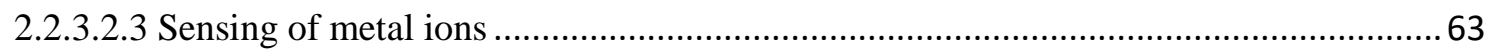

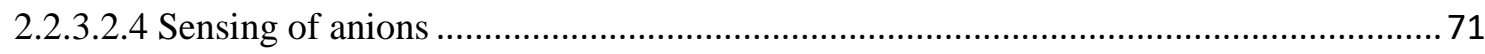

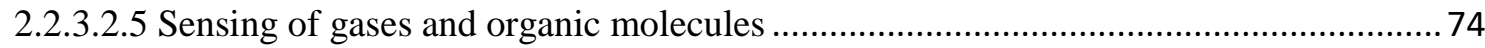

3. Anti-Stokes Shift Luminescent Metal-organic Frameworks............................................................. 80

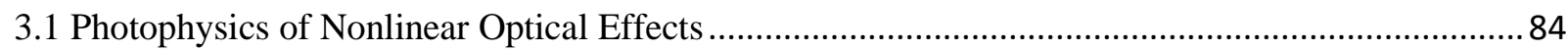

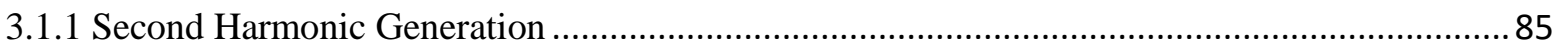

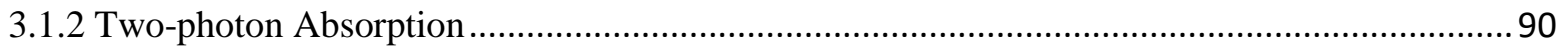

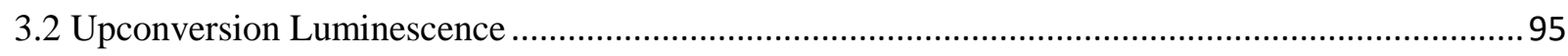

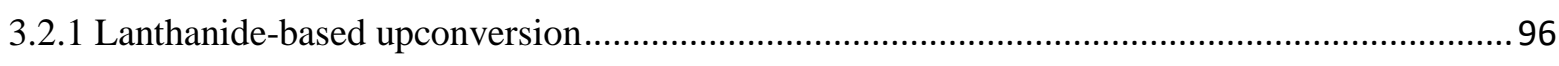

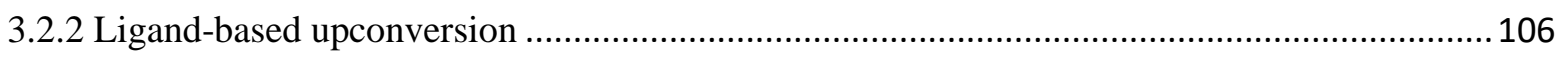

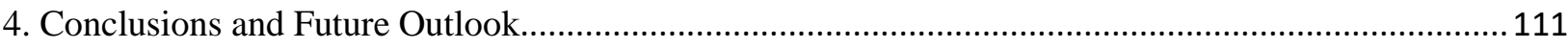

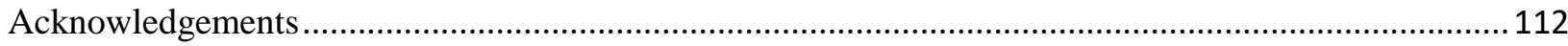

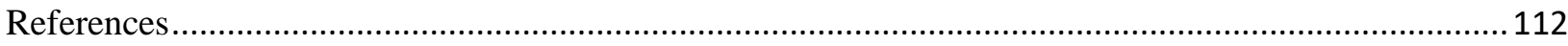




\section{Introduction}

Light is an electromagnetic radiation, with wavelengths in the optical range (100 $\mathrm{nm}-1 \mathrm{~mm})$ of the electromagnetic spectrum. Visible (Vis) light has wavelengths between 400 and 700 $\mathrm{nm}$ and can be seen by human eyes. Light with wavelengths shorter than $400 \mathrm{~nm}$ falls in the ultraviolet (UV) region, which is further divided into UV-A, UV-B, and UV-C ranges. Similarly, light with wavelengths longer than $700 \mathrm{~nm}$ are infrared (IR) radiations; this region is also subdivided into IR-A, IR-B and IR-C ranges.

What happens when light interacts with matter? The answer to this question depends on the nature of the material and the wavelength of the light. For example, at the surface of a rough wall, light is scattered; through a green leaf, light is partially absorbed and partially reflected; whilst through an interface between two transparent media such as glass and water, light is refracted. Light with shorter wavelengths are scattered much more than the light with longer wavelengths. The blue color of the sky is a typical example of light scattering, mainly for the shorter wavelengths. Without the scattering, the sky would appear black to our eyes.

Some materials not only absorb light but also emit light with a longer wavelength; this phenomenon is called photoluminescence. There are also materials that absorb longwavelength light and emit light with a shorter wavelength; due to nonlinear interactions with light or the upconversion of luminescence. These light-emitting phenomena are quite fascinating and the materials emitting light can be employed in numerous applications such as in lighting, imaging, sensing, or solar light harvesting. While photoluminescence is more popular and can be observed in many organic and inorganic materials, in proteins and quantum dots, upconversion of luminescence is rather rare and mainly observed in lanthanide-doped nanoparticles. Recently, there is a growing interest in studying these lightemitting phenomena on a relatively new and unique class of materials: metal-organic frameworks (MOFs). 
MOFs are a class of crystalline microporous materials that were initially introduced in the 1990s [1], which comprise metal ions or clusters linked by organic ligands via coordination bonds forming 1-, 2- or 3-dimensional networks. MOF materials often exhibit high porosities, with several MOFs displaying the highest volume and surface area, and the lowest crystal density among all porous materials known to date [2, 3]. Another fascinating feature of MOFs is their high structural tunability, i.e. by changing the metal ion and/or the organic ligand, different structures with a variety of properties can be synthesized [4, 5]. This makes MOFs strikingly different from other porous solids such as zeolites, silica, or activated carbon, and allows them to be used in a wide range of applications.[6-8] Besides being excellent candidates for gas capture, storage and separation [9-14], MOFs have also been employed in areas such as catalysis [15-20], sensing [21-23], nonlinear optics [24], ferroelectricity [25], magnetism [26], electrical conductivity [27-30], and drug delivery [3134]. In recent years, light-emitting MOFs have been in the limelight and in fact, there have already been many great reviews on photoluminescent and nonlinear optical MOFs [24, 35-39]. While these reviews have excellently provided the current status of the research on lightemitting MOFs, we believe that a tutorial review providing fundamental photophysical principles of luminescence and nonlinear optical effects, and focusing on mechanistic aspects of each application of these materials would be valuable to a general audience and will be an important addition to the other reviews. In the following sections, we discuss in detail the mechanisms and important parameters of photoluminescence and nonlinear optics, the applications of light-emitting MOFs, and then conclude with our visions about the field in the near future. 


\section{Photoluminescent Metal-organic Frameworks}

\subsection{Photophysics of Photoluminescence}

Luminescent materials are compounds that emit light, mostly in the visible and near infrared (NIR) regions, through the conversion of the excitation energy into an electromagnetic radiation. The light emission can be caused by many types of excitation energies such as the energy of a chemical reaction (chemiluminescence), the energy when passing an electric current through a substance (electroluminescence), the energy as a result of a mechanical action (mechanoluminescence), or the energy due to absorption of photons (photoluminescence). In this section, we will discuss photoluminescence (hereafter luminescence) and photoluminescent (hereafter luminescent) materials based on organic, transition metal and lanthanide compounds and quantum dots, although photoluminescence has also been observed in proteins [40] and inorganic pigments [41]. For an exhaustive technical description of other types of luminescence, readers are directed to several articles that have been authored on the topic [42-44].

Luminescence is often divided into two categories-fluorescence and phosphorescencedepending on the nature of the excited state. The mechanism of these two phenomena can be illustrated by a Jablonski diagram shown in Figure 1. Fluorescence occurs when electrons in excited singlet states $\left(\mathrm{S}_{1}\right)$ return to the ground state $\left(\mathrm{S}_{0}\right)$ via photon emission. Since both the excited state and the ground state have the same spin, the process is spin allowed according to the spin selection rule; hence, fluorescence occurs rapidly with the typical lifetime in the ns range. Phosphorescence involves the relaxation of electrons from triplet excited states $\left(\mathrm{T}_{1}\right)$, which can be populated via intersystem crossing from the singlet excited states. Transitions between states with different spins are spin forbidden; therefore, phosphorescence lifetimes are typically in the range of $\mu \mathrm{s}-\mathrm{ms}$ and even up to $\mathrm{s}$ or minutes. Accordingly, phosphorescence continues even after the radiation causing it has ceased. In addition, 
phosphorescence emission from $\mathrm{T}_{1}$ is generally shifted to longer wavelengths (lower energy) relative to the fluorescence (Figure 1). It is interesting to note that several luminescent compounds were misleadingly named; for example, white phosphorous - from which the term "phosphorescence" originally derived, does not actually exhibit phosphorescence, its ability to exhibit green glow in the dark upon exposure to oxygen is due to a chemiluminescent mechanism.

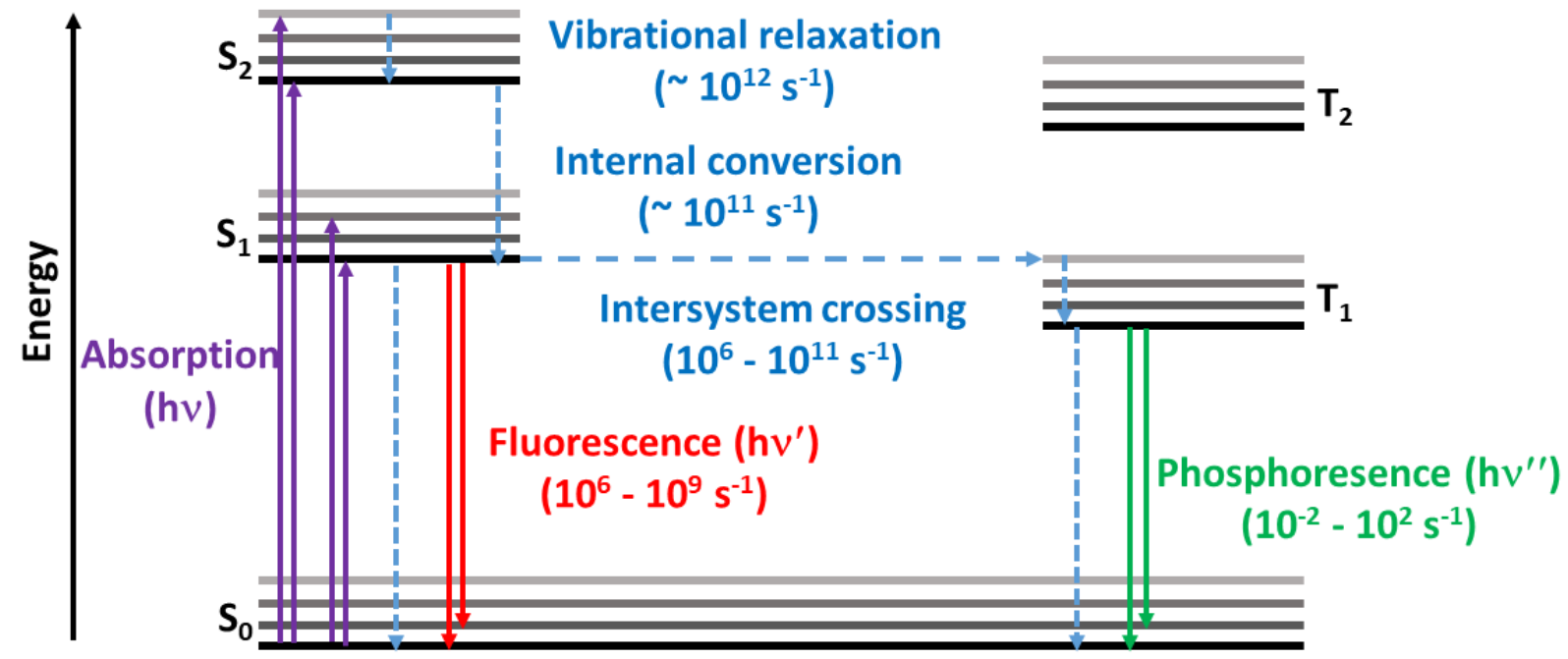

Figure 1. A Jablonski diagram illustrating light absorption and emission processes. The rate of excited state decay is of $10^{6}-10^{9} \mathrm{~s}^{-1}$ for fluorescence and $10^{-2}-10^{2} \mathrm{~s}^{-1}$ for phosphorescence.

The important characteristics for luminescent materials include the quantum yield $(\phi)$, molar absorptivity $(\varepsilon)$ (also called molar extinction coefficient), luminescence lifetime $(\tau)$, and Stokes shift. Quantum yield is the ratio of the number of emitted photons to the number of absorbed photons. In other words, quantum yield gives the measure of the efficiency to convert the absorbed photon energy into light; therefore, it has been a key criterion for the selection of luminescent materials in many applications such as laser and lighting. Direct 
measurement of quantum yield as an absolute quantity can be obtained by using an integrating sphere but this method is often challenging and requires sophisticated instruments; the more common method (but less reliable) for determination of quantum yield is by comparison with a standard of known quantum yield, e.g. quinine bisulfate, fluorescein, or rhodamine $6 \mathrm{G}$. The quantum yields of these standards are mostly independent of excitation energy; hence, these compounds can be used in a wide range of wavelengths as long as they have effective absorption. Most materials exhibit quantum yields smaller than unity due to non-radiative transitions, e.g. internal conversions, intersystem crossing, and vibrational transitions (Figure 1), which compete with the radiative transitions and give up energy to the surroundings. These non-radiative transitions can be very sensitive to the local environment; therefore, the quantum yield can sometimes be used as a probe of environment near the luminescent molecules. Several organic compounds such as rhodamines and BODIPY dyes exhibit near-unity quantum yields and are very bright luminophores. Most quantum dots display high quantum yields of $\sim 50 \%$, whereas luminescent transition metal complexes exhibit lower quantum yields, e.g. the quantum yield of $\left[\mathrm{Ru}(\mathrm{bpy})_{3}\right]^{2+}\left(\mathrm{bpy}=2,2^{\prime}\right.$-bipyridine $)$ in aqueous solution is $\sim 4.2 \%$ [45]. For lanthanide compounds, the total quantum yield varies depending on the nature of the lanthanide ions; furthermore, it is strongly affected by multiphonon relaxations, particularly for those emitting in the NIR region, and the efficiency of the energy transfer process between the organic ligands and the lanthanide ions (vide infra).

Molar absorptivity measures how well a luminophore absorbs a given wavelength of light and is one of the two factors along with quantum yield contributing to the luminescence intensity of the material. Ordinary (one-photon) absorption follows the Lambert-Beer law 
(equation 1), which relates the absorbance of the material sample to its molar absorptivity, concentration, and thickness,

$A=\log \frac{I}{I_{0}}=\varepsilon l C$

where $A$ is the absorbance of the sample; $I_{0}$ and $I$ are the intensity of light entering and emerging from the sample, respectively; $\varepsilon$ is the molar absorptivity; $l$ is the thickness of the sample; and $C$ is the sample concentration. It is worth noting that deviations from the Lambert-Beer's law can occur in solutions of aggregates or macromolecules due to light scattering. Most organic dyes and luminescent transition metal complexes absorb light efficiently, with $\varepsilon$ in the range of $10^{5}-10^{6} \mathrm{M}^{-1} \mathrm{~cm}^{-1}$. The molar absorptivity of quantum dots is also in the $10^{5}-10^{6} \mathrm{M}^{-1} \mathrm{~cm}^{-1}$ range but it is strongly dependent on the size of the particles [46]. On the other hand, lanthanide ions themselves are poor absorbers, with $\varepsilon$ often lower than $10 \mathrm{M}^{-1} \mathrm{~cm}^{-1}$, resulting in low luminescence intensity. When a chromophore with a high molar absorptivity is attached to the lanthanide complex, the photon energy absorbed can be transferred from the chromophore to the lanthanide ion, which significantly improves the overall brightness of these complexes. This phenomenon is called the "antenna effect" and will be discussed in the following part of this section.

Luminescence lifetime is the average time the luminophore stays in its excited state before emitting a photon and is mathematically expressed as the time required for the luminescence intensity to decay to $1 / \mathrm{e}(\sim 37 \%)$ of the original intensity (equation 2$)$,

$I_{t}=I_{0} e^{-t / \tau}$

where $I_{\mathrm{t}}$ and $I_{0}$ are luminescence intensity at time $\mathrm{t}$ and $\mathrm{t}=0$, respectively; and $\tau$ is the lifetime. In practice, lifetime can be measured by either a time-domain or a frequency-domain technique, with the former often providing the estimate of $\tau$ with lower uncertainty [47]. Generally, fluorescence lifetimes of organic chromophores are $<10 \mathrm{~ns}$, e.g. fluorescein 
isothiocyanate has a lifetime of $3.9 \mathrm{~ns}$ at $\mathrm{pH}=7.2$, with several exceptions such as pyrene and coronene displaying lifetimes > $100 \mathrm{~ns}$. Quantum dots display lifetimes of dozens of ns while lanthanide compounds typically exhibit much longer lifetimes in the $\mu \mathrm{s}-\mathrm{ms}$ range due to the forbidden nature of $\mathrm{f}-\mathrm{f}$ transitions. Luminescent transition metal complexes of $\mathrm{d}^{6}, \mathrm{~d}^{8}$, and $\mathrm{d}^{10}$ metals are another class of long-lifetime emitters, which can display lifetimes of few hundreds ns[48, 49]. Similar to quantum yield, the lifetime of a luminophore highly depends on the local environment but is independent of its concentration; therefore, measuring the lifetime can provide a sensitive measure of local conditions, especially for uses when luminophores might not be evenly distributed, e.g. in biological cells or tissues. For practical applications, lifetime is an important characteristic of luminescence materials as it determines the timescale of experiments. For example, in conventional fluoroimmunoassays, background fluorescence from serums, solvents, cuvettes, and the sample has been one of the major problems. When a long-lived luminophore is used to illuminate cells or tissues, by delaying the measurement of fluorescence after a flash excitation of the sample, i.e. time-resolved experiment, all background fluorescence is excluded and the only background signal observed is due to nonspecific binding of the tracer.

Stokes shift, named after the physicist George G Stokes, is the difference between the peak excitation and the peak emission wavelengths of the same electronic transition. If the excitation and emission of the material are not from the same electronic transition, the shift is called pseudo Stokes shift. In ordinary cases with one-photon absorption, the peak emission is almost always at longer wavelength (lower energy) than the one of excitation. Organic compounds typically display small Stokes shifts $(<100 \mathrm{~nm})$, e.g. cyanine dyes such as Cy3 and Cy5 can be excited at wavelengths of $550 \mathrm{~nm}$ and $650 \mathrm{~nm}$ and emit fluorescence at 570 $\mathrm{nm}$ and $670 \mathrm{~nm}$, respectively. In addition, the magnitude of these organic-fluorophore Stokes shifts also depends on the polarity of the solvent; the more polar solvent often leads to larger 
Stoke shifts due to the ability of these solvent molecules to reorient themselves in the proximity of the fluorescent molecules to compensate for the dipole moment of the fluorophore after photoexcitation and as a consequence, fluorescence emits from a state of lower energy. Luminescent transition metal complexes display relatively large Stokes shifts, e.g. $\left[\mathrm{Ru}(\mathrm{bpy})_{3}\right]^{2+}$ can be excited at $\sim 450 \mathrm{~nm}$ and emits phosphorescence at $\sim 620 \mathrm{~nm}[50]$. Lanthanide compounds exhibit large pseudo Stokes shift, e.g. Er ${ }^{\mathrm{III}}$ ion displays NIR emission at $\sim 1550 \mathrm{~nm}$, hence the Stokes shift is of $\sim 1100 \mathrm{~nm}$ if the compound is excited at $400 \mathrm{~nm}$. For quantum dots, the emission wavelength is dependent on the dimensions of the particles; therefore their Stokes shifts can be tuned by controlling the size of the dots. Stokes shift is important not only for luminescent measurements, since the redshift allows the use of appropriate optical filters to block the excitation light from reaching the detector so that fluorescence detection is measured against a very low background, but also for many practical applications. For example, fluorescent organic dyes have been utilized in luminescent solar contractor (LSC) to produce electricity from solar radiation. Dyes with large Stoke shift are ideal for this application since the re-absorption of the emitted light due to the overlap of absorption and emission significantly limits the light collecting efficiency of the device [51].

Among the luminescent materials, organic dyes are probably the most widespread fluorophores and many are commercially available. Organic dyes can be divided into resonant dyes and charge-transfer (CT) dyes, of which the fluorescence emission originates from an electronic state delocalized over the whole chromophore for the former and from intramolecular charge-transfer transitions for the latter. CT dyes often exhibit lower molar absorptivities and fluorescence quantum yields compared to resonant dyes, and display a strong solvent-polarity dependence of their photophysical properties in solution. The most 
common resonant organic fluorophore families are fluoresceins/rhodamines, BODIPYs, indocyanines, porphyrines, and phthalocyanines [52], whilst coumarins and dansyl fluorophores are representatives for CT dyes. Despite the great availability, moderate-to-high quantum yields, and large molar absorptivity, organic dyes exhibit several serious drawbacks compared to quantum dots and transition metal and lanthanide compounds. Besides the above-mentioned short lifetimes and small Stokes shifts, which often requires significant efforts to disentangle the absorption and emission processes in practical applications, many organic dyes exhibit fast photobleaching caused by the cleaving of covalent bonds or nonspecific reactions between the fluorophore and surrounding environment leading to the complete loss of fluorescence in a short period of time. Although some organic dyes such as the Alexa dyes have been designed to enhance the photostability in comparison to the firstgeneration fluorophores such as fluorescein[53], their uses are still limited to applications with low excitation-light intensities and in short terms. Anti-fade reagents can be used to reduce photobleaching and preserve the fluorescence signal intensity over longer period of time[54]; however, this extra step is rather inconvenient for practical usages. For applications in biological fluorescent imaging, due to the high hydrophobicity of most organic dyes, chemical modifications to increase water solubility are often needed; for example, modifications with sulfonate or saccharide functions to improve water solubility have been proposed $[55,56]$.

Transition metal compounds of $\mathrm{d}^{6}, \mathrm{~d}^{8}$ and $\mathrm{d}^{10}$ metals exhibiting luminescence have been known since the 70 's of the last century, beginning with the $\mathrm{d}^{6}$ complexes of $\mathrm{Ru}^{\mathrm{II}}$ polypyridine, e.g. $\left[\mathrm{Ru}(\mathrm{bpy})_{3}\right]^{2+}[57]$. In contrast to organic dyes, which are mostly singlet emitters and display fluorescence, these complexes exhibit phosphorescence emission due to the metal-to-ligand charge transfer (MLCT) involving the oxidation of $\mathrm{Ru}^{\mathrm{II}}$ to $\mathrm{Ru}^{\mathrm{III}}$ and the 
reduction of the polypyridine ligand (Figure 2). Similar bpy-based complexes with Os ${ }^{\mathrm{II}}$ have also been extensively studied [58], which display lower emission energy and shorter luminescence lifetime due to the lower energy gap between the $\mathrm{d} \pi$ orbital of Os ${ }^{\mathrm{II}}$ and the $\pi^{*}$ orbital of the polypyridine ligand. $\operatorname{Re}^{\mathrm{I}}$ tricarbonyl polypyridines, e.g. $\left[\operatorname{Re}(\mathrm{CO})_{3}(\mathrm{bpy})\right]^{+}$, are another $\mathrm{d}^{6}$ MLCT system that attracts wide interest since these complexes display intense and long-lived luminescence [59-61]. Ir ${ }^{\mathrm{III}}$ polypyridine complexes are also known for a long time [62] but most studies on these complexes have exploited the catalytic activity of the $\operatorname{Ir}^{\mathrm{III}}$ ion rather than the photophysical property. Recently, numerous luminescent Ir $^{\mathrm{III}}$ polypyridine complexes have been synthesized and studied [63-65]. Interestingly, several Ir ${ }^{\mathrm{III}}$ polypyridine complexes emit phosphorescence not originated from the MCLT states but rather from ligand-to-ligand charge-transfer (LLCT) [66] and sigma-bond-to-ligand charge-transfer (SBLCT) [67] states that were observed in these systems. For $\mathrm{d}^{8}$ luminescent complexes, $\mathrm{Pt}^{\mathrm{II}}$ terpyridines, e.g. $[\operatorname{Pt}(\text { terpy }) \mathrm{L}]^{\mathrm{n}+}$, have been extensively studied mainly due to their potential applications as biological probes and as antitumor drugs $[68,69]$. The nature of the ligand $\mathrm{L}$ significantly affects the luminescence emission of the $\mathrm{Pt}^{\mathrm{II}}$ ion; for example, the complex $[\mathrm{Pt}(\text { terpy }) \mathrm{Cl}]^{+}$is non-emissive in solution at room temperature due to the presence of a lowenergy d-d ligand field whereas the complexes $[\mathrm{Pt}(\text { terpy })(\mathrm{C} \equiv \mathrm{CR})]^{+}$exhibit intense luminescence in the same condition. In addition, the complexes with the electron-rich $\mathrm{R}$ groups exhibit low emission energies while those with electron-poor groups display high emission energies, which is consistent with the MLCT mechanism [70]. Luminescent $\mathrm{d}^{10}$ complexes of $\mathrm{Cu}^{\mathrm{I}}, \mathrm{Ag}^{\mathrm{I}}$ and $\mathrm{Au}^{\mathrm{I}}$ have also been reported in the literature [69]. The $\mathrm{d}^{10}$ metals, particularly $\mathrm{Au}^{\mathrm{I}}$, display strong non-covalent metal-metal interactions, i.e. aurophilicity [71], and often form complexes containing more than one metal atom. Large complexes such as dodecanuclear, and octadecanuclear $\mathrm{Au}^{\mathrm{I}}$ sulfide clusters have been known to exhibit orange to red luminescence, which is often attributed to the triplet ligand-to-metal charge-transfer 
(LMCT) states mixed with metal-centered states modified by $\mathrm{Au}^{\mathrm{I}} \cdots \mathrm{Au}^{\mathrm{I}}$ interactions $[72,73]$. Near-infrared (NIR) luminescent emission was also observed in $\mathrm{Au}^{\mathrm{I}}$ selenide clusters although the origin of such low energy emission is not clear [74]. Overall, with high quantum yields and relatively long lifetimes, luminescent transition metal complexes have enjoy widespread promising applications in sensing [75-77], biological imaging [78, 79], organic light-emitting diodes (OLEDs) [80-84], and renewable energy [85-91].

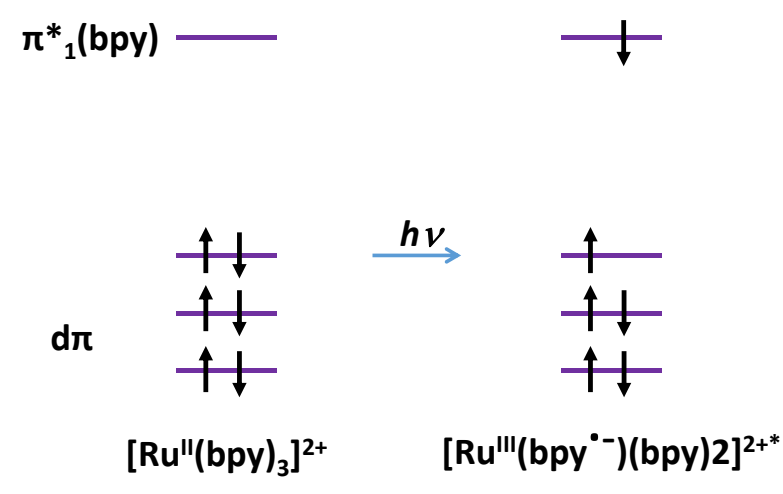

Figure 2. Metal-to-ligand charge transfer $\mathrm{d} \pi^{6} \rightarrow\left(\mathrm{d} \pi^{5} \pi^{* 1}\right)$ upon photon absorption.

Semiconductor quantum dots (QDs) are nanometer-scale particles, which were first reported by Brus and coworkers in 1982 [92]. QDs contain atoms of group II and VI elements (e.g. $\mathrm{CdSe}, \mathrm{CdTe}$ ) or group III and $\mathrm{V}$ elements (e.g. InP, InAs, InGaP) with the size ranging from 2 - $10 \mathrm{~nm}$. Due to their nanoscale sizes, QDs possess properties between bulk materials and molecules [93, 94]. When QDs are excited by photon energy, the separation between the excited electron and hole is smaller than their Bohr radius; hence, the exciton is confined in a small space with high energy leading to fluorescence emission. Consequently, QDs emit intermittent fluorescence when absorbing photons with energies higher than the band gap. The emission wavelength is dependent on the size of the QDs, with larger QDs exhibiting a smaller energy band-gap and red fluorescence, whereas smaller QDs emit blue fluorescence (Figure 3). This effect allows for tuning the colors of QDs by controlling their size, with their 
emission covering the spectral range from the ultraviolet (UV) to the infrared (IR) having been reported in the literature [95-97]. Although QDs exhibit short luminescence lifetimes (ns) like organic dyes, one advantage of using QDs is their insusceptibility to photobleaching [98, 99]. For example, QDs have shown to be 100 times more stable and 20 times brighter than Rhodamine 6G [100]. However, due to the surface irregularities of the core structure, emission irregularities like blinking are often observed. Another significant drawback of QDs, especially for those containing $\mathrm{Cd}^{\mathrm{II}}$, is their high reactivity and toxicity [101], which might limit their practical applications. Capping the core of QDs with a high-bandgap semiconducting materials, e.g. $\mathrm{ZnS}$, can improve their luminescence properties and decrease the release of toxic ions such as $\mathrm{Cd}^{2+}, \mathrm{Se}^{2-}$ or $\mathrm{Te}^{2-}$ into the environment $[102,103]$. For biological applications, a second coating with silica [104], phospholipid micelles [105], or oligomeric ligands [106] is required to ensure solubility and stability in biological media.

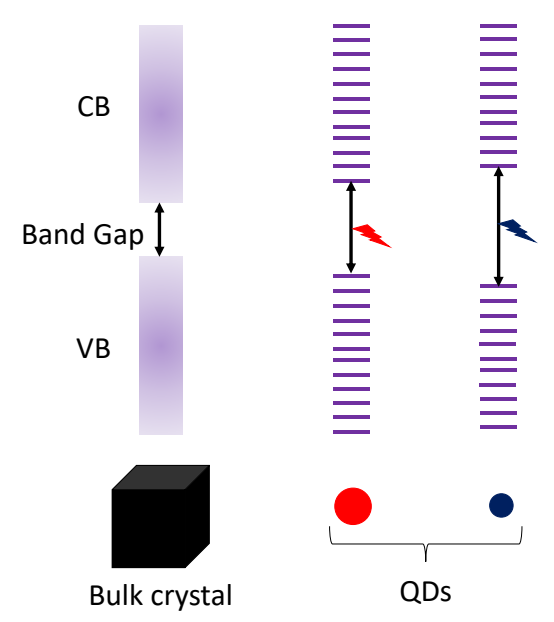

Figure 3. Schematic illustration of the band gap of bulk crystal and QDs.

Luminescence emission from lanthanide compounds has been the subject of research for nearly a century, with some of the early articles describing the complexity of lanthanide optical spectra back in the 30's [107]. Lanthanide ions have the general electron configuration of $[\mathrm{Xe}] \mathrm{f}^{\mathrm{n}}(\mathrm{n}=0-14)$ and the ionic radii decrease along the series from La $\mathrm{III}^{\mathrm{III}}$ 
$\left([\mathrm{Xe}] \mathrm{f}^{0}\right)$ to $\mathrm{Lu}^{\mathrm{III}}\left([\mathrm{Xe}] \mathrm{f}^{14}\right)$, which in some cases leads to a change in the coordination number from nine in large lanthanides to eight in smaller ones. The $4 \mathrm{f}$ orbitals are "inner orbitals" due to the shielding by the Xenon core and the larger radial expansion of the $5 s^{2} 5 p^{6}$ subshells; therefore, $4 \mathrm{f}$ electrons are not involved in bonding and most lanthanide ions in complexes behave similarly as free ions, which is important for the spectroscopic properties of these ions. Luminescence emission of lanthanide ions originates from f-f transitions (except for $\mathrm{Ce}^{\mathrm{III}}$ ), which involve a rearrangement of the electrons in the $4 \mathrm{f}$ subshell. Except for $\mathrm{La}^{\mathrm{III}}$ and $\mathrm{Lu}^{\mathrm{III}}$, which have zero and completely filled $\mathrm{f}$ electrons and hence no $\mathrm{f}$ - $\mathrm{f}$ transitions, all other lanthanide ions are luminescent and the emission wavelength ranges from $\mathrm{UV}\left(\mathrm{Gd}^{\mathrm{III}}\right)$ to visible (blue $\mathrm{Tm}^{\mathrm{III}}$, green $\mathrm{Tb}^{\mathrm{III}}$, orange $\mathrm{Sm}^{\mathrm{III}}$, red $\left.\mathrm{Eu}^{\mathrm{III}}\right)$ to NIR (Pr $\left.{ }^{\mathrm{III}}, \mathrm{Nd}^{\mathrm{III}}, \mathrm{Er}^{\mathrm{III}}, \mathrm{Yb}^{\mathrm{III}}\right)$. Dy ${ }^{\mathrm{III}}$ exhibits luminescence in both visible and NIR regions while $\mathrm{Ce}^{\mathrm{III}}$ luminescence emission is based on a $5 \mathrm{~d}-4 \mathrm{f}$ transition and is hence strongly affected by the environment around the Ce $\mathrm{e}^{\mathrm{III}}$ ion due to the strong crystal-field effects on the $5 \mathrm{~d}^{1}$ excited state. Since $\mathrm{f}$ - $\mathrm{f}$ transitions are spin-parity forbidden according to the Laporte's rule, they display faint intensities, negligible Stokes shifts, long-lifetime emission, sharp emission lines, and insensitivity to the environment around the lanthanide ion. The last three characteristics make lanthanide luminescence easily recognized and therefore lanthanide ions are ideal candidates for optical probes; the first two characteristics can be significantly improved by employing the "antenna effect". To increase luminescence intensity, lanthanide compounds can be modified with one or several organic chromophores, which often have large molar absorptivities $\left(\varepsilon>10^{5} \mathrm{M}^{-1}\right.$ $\mathrm{cm}^{-1}$ ), to play the role of antennas for the lanthanide ions. These antennas can efficiently absorb photon energy and transfer to the lanthanide emitting states to induce luminescence emission. The overall mechanism of lanthanide luminescence therefore involves light absorption, energy transfer, and light emission processes (Figure 4) and become quite complex with several mechanisms and energy levels associated [108]. The main energy 
migration path after light absorption is believed to involve an intersystem-crossing from the singlet excited state $S_{1}$ to the long-lived ligand-centered triplet state $T_{1}$, from which energy transfer occurs (Figure 4, right). The direct energy transfer from the $\mathrm{S}_{1}$ state is also possible but with lower probability due to the short lifetime of $S_{1}$. Two mechanisms of energy transfer were proposed and theoretically modeled: a double-electron exchange (Dexter) and a dipoledipole (Förster) mechanism [109]. The former occurs when the chromophore ligands are in close distance to the lanthanide ions while the latter operates even with the distance between them of up to $\sim 10 \mathrm{~nm}$, i.e. through-space energy transfer. The efficiency of the Förster energy transfer is inversely proportional to the sixth power of the distance between the chromophores and the lanthanide ions. The overall luminescence quantum yield of a lanthanide compound depends on the efficiencies of the intersystem-crossing and the energytransfer processes, and on the intrinsic quantum yield of the lanthanide ion, as shown in equation 3 ,

$\phi_{L}^{L n}=\eta_{I S C} \eta_{E T} \phi_{L n}^{L n}$

where $\phi_{L}^{L n}$ and $\phi_{L n}^{L n}$ are the total and the intrinsic quantum yields, respectively; and $\eta_{I S C}$ and $\eta_{E T}$ are the intersystem-crossing and energy-transfer efficiencies, respectively. It is worth mentioning that besides the increasing of luminescence intensity, large "pseudo" Stokes shifts are also observed as the consequence of the antenna effect.

Non-radiative multiphonon relaxation of lanthanide luminescence caused by the presence of high-energy $\mathrm{C}-\mathrm{H}, \mathrm{O}-\mathrm{H}$, and $\mathrm{N}-\mathrm{H}$ vibrators near by the lanthanide ion, is a major concern in the design of highly luminescent lanthanide compounds [110-112]. Coordinating solvent molecules such as $\mathrm{H}_{2} \mathrm{O}$ and $\mathrm{MeOH}$ significantly quench luminescent emission leading to low quantum yields and short lifetimes, particularly for lanthanide ions emitting in the NIR region. Besides efforts to design lanthanide compounds that do not contain coordinating 
solvents such as a recent report by our group [113], attempts to minimize the C-H quenching in NIR lanthanide complexes by utilizing fully fluorinated ligands were also performed [114116]. Ligand-to-metal distance is another parameter that needs to be optimized since a too short distance can give rise to luminescent quenching while a large distance leads to poor electron transfer and reduced luminescent intensity. Several recent studies demonstrated that by placing a lanthanide ion within diamagnetic metallacrowns, the lanthanide center is well protected from coordinating solvents while at the same time the ligands are at an optimum distance to the lanthanide ion leading to high quantum yields and long lifetimes [117-120].
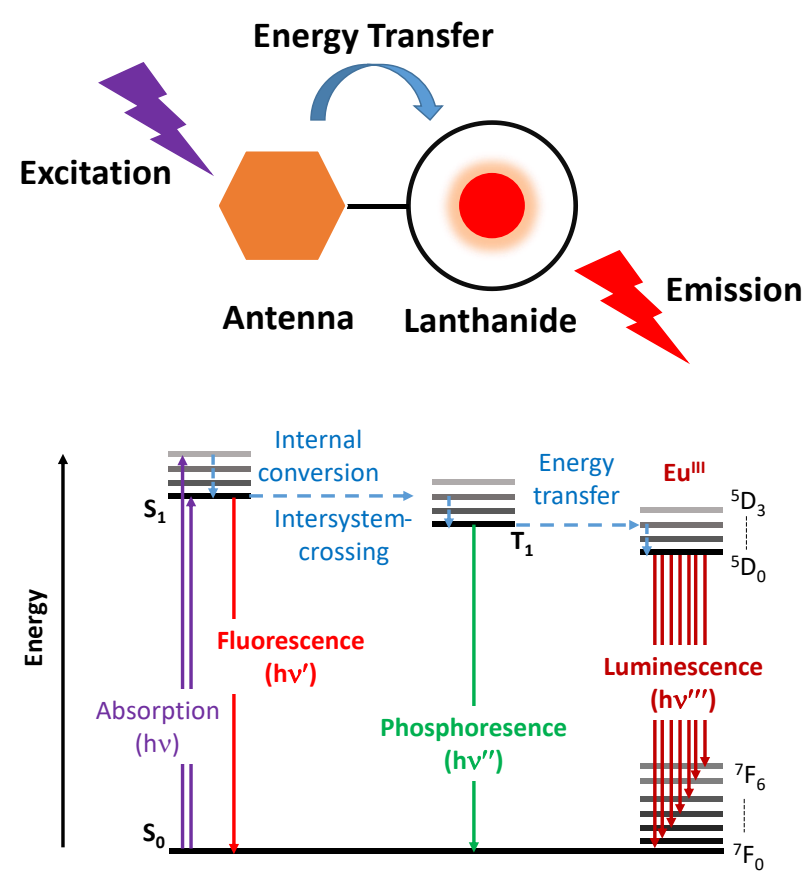

Figure 4. Mechanism of lanthanide luminescence (left) and a simplified Jablonski diagram illustrating the electronic states, the energy-transfer pathways, and the transitions within a $\mathrm{Eu}^{\mathrm{III}}$ complex (right).

\subsection{Applications of Luminescent Metal-Organic Frameworks}

MOFs exhibiting luminescence are promising for a wide range of applications such as whitelight emission, biological fluorescent imaging, optical thermometry and chemical sensing. 
Development of MOFs as solid-state luminescent materials takes advantage of their structural predictability, high crystallinity, high porosity, and reusability compared to traditional luminescent compounds. Luminescent MOFs can be designed by tailoring their structures with fluorescent organic ligands or lanthanide ions, or by introducing guest molecules such as organic dyes, transition metal complexes, or quantum dots into their frameworks. This synthetic flexibility of the organic and inorganic components, the host framework, and the voids within MOFs offer ample possibilities for tuning their photophysical properties. A complete list of luminescent MOFs has been provided in several previous review articles [35$38,54,121-127]$. The scope of this section is not about giving a summary of all reported luminescent MOFs but to discuss the potential applications of luminescent MOFs with the focus being the fundamental principles and some recent interesting studies in each application. The list of MOFs reviewed in this part is provided in Table 1.

\begin{tabular}{|c|c|c|c|c|}
\hline & MOF & $\begin{array}{l}\text { Organic } \\
\text { Luminophore/ } \\
\text { Ligand }\end{array}$ & Photophysical Properties & $\begin{array}{c}\text { Ref } \\
\text { (Year) }\end{array}$ \\
\hline & White-light Emission & & & \\
\hline 1 & $\begin{array}{l}{\left[\mathrm{Cd}(\text { tzphtpy })_{2}\right]} \\
\text { Htzphtpy }=4 \text {-(tetrazol-5-yl)phenyl- } \\
2,2^{\prime}: 6^{\prime}, 2^{\prime \prime} \text {-terpyridine }\end{array}$ & & $\begin{array}{l}\lambda_{\mathrm{ex}}=386 \mathrm{~nm} \\
\Phi=2.3 \%\left(\lambda_{\mathrm{ex}}=326 \mathrm{~nm}\right) \\
\tau_{1}=9.89 \mathrm{~ns}, \tau_{2}=8.26 \mathrm{~ns} \\
\text { CIE coordinates: }(0.33,0.36) \\
\text { CIE } R_{a}=77, \text { CCT }=5328 \mathrm{~K}\end{array}$ & $\begin{array}{l}{[128]} \\
(2016)\end{array}$ \\
\hline 2 & $\begin{array}{l}{[\mathrm{PbL}]} \\
\left.\mathrm{H}_{2} \mathrm{~L}=2,5 \text {-bis ((2-hydroxylpropyl }\right) \\
\text { thio)-1,4-benzene dicarboxylic acid }\end{array}$ & $100^{\Theta}$ & $\begin{array}{l}\lambda_{\mathrm{ex}}=300,350, \text { and } 400 \mathrm{~nm} \\
\Phi=2-3 \% \\
\text { CIE coordinates: }(0.27,0.30) \\
(0.25,0.29) ;(0.24,0.28) \text { for }\end{array}$ & $\begin{array}{l}\text { [129] } \\
(2012)\end{array}$ \\
\hline
\end{tabular}




\begin{tabular}{|c|c|c|c|c|}
\hline & & & respective excitation wavelength & \\
\hline 3 & $\begin{array}{l}{[\mathrm{AgL}]} \\
\mathrm{HL}=4 \text {-cyanobenzoate }\end{array}$ & $\begin{array}{l}0 \\
0 \\
0\end{array}$ & $\begin{array}{l}\lambda_{\mathrm{ex}}=349 \text { and } 350 \mathrm{~nm} \\
\Phi=10.86 \%\left(\lambda_{\mathrm{ex}}=349 \mathrm{~nm}\right) \\
\text { CIE coordinates: }(0.33,0.34) \text { and } \\
(0.31,0.33) \text { for respective } \\
\text { excitation wavelength }\end{array}$ & $\begin{array}{l}{[130]} \\
(2009)\end{array}$ \\
\hline 4 & $\begin{array}{l}\text { Eu-doped }\left[\operatorname{In}(\mathrm{BTB})_{2 / 3}(\mathrm{OA})(\mathrm{DEF})_{3 / 2}\right] \\
\mathrm{H}_{3} \mathrm{BTB}=4,4^{\prime}, 4^{\prime \prime} \text {-benzene-1,3,5- } \\
\text { triyl-tris(benzoic acid) } \\
\mathrm{H}_{2} \mathrm{OA}=\text { oxalic acid } \\
\text { DEF = diethylformamide }\end{array}$ & & $\begin{array}{l}\lambda_{\mathrm{ex}}=350,360,380,394 \mathrm{~nm} \\
\Phi=4.3 \%\left(\lambda_{\mathrm{ex}}=330 \mathrm{~nm}\right) \\
\text { CIE coordinates: }(0.369,0.301), \\
(0.309,0.298),(0.285,0.309), \\
\text { and }(0.304,0.343) \text { for respective } \\
\text { excitation wavelength } \\
\text { CIE } R_{a}=90, \mathrm{CCT}=3200 \mathrm{~K}\end{array}$ & $\begin{array}{l}{[131]} \\
(2012)\end{array}$ \\
\hline 5 & $\begin{array}{l}{\left[\mathrm{Eu}_{0.3} \mathrm{~Tb}_{2.7}(\mathrm{BDC})_{4.3875}(\mathrm{~L})_{0.1125}(\mathrm{~S})_{\mathrm{a}}\right]} \\
\mathrm{H}_{2} \mathrm{BDC}=\text { terephthalic acid } \\
\mathrm{H}_{2} \mathrm{~L}=2 \text {-(pyrene-1-carboxamido) } \\
\text { terephthalic acid } \\
\mathrm{S}=\text { solvent }\end{array}$ & & $\begin{array}{l}\lambda_{\mathrm{ex}}=304 \mathrm{~nm} \\
\text { CIE coordinates: }(0.302,0.342)\end{array}$ & $\begin{array}{l}{[132]} \\
(2016)\end{array}$ \\
\hline 6 & $\begin{array}{l}{\left[\left(\mathrm{Eu}_{\mathrm{x}} \mathrm{Tb}_{\mathrm{y}} \mathrm{Dy}_{1-\mathrm{x}-\mathrm{y}}\right)(\mathrm{HL})\left(\mathrm{H}_{2} \mathrm{O}\right)(\mathrm{DEF})\right]} \\
(1) \mathrm{x}=0.0667, \mathrm{y}=0.0667, \mathrm{z}=0.8666 \\
(2) \mathrm{x}=0.0666, \mathrm{y}=0.4667, \mathrm{z}=0.4667 \\
\mathrm{H}_{4} \mathrm{~L}=\text { p-terphenyl-2.2",4,4"- } \\
\text { tetracarboxylic acid }\end{array}$ & & $\begin{array}{l}\lambda_{\mathrm{ex}}=336 \mathrm{~nm} \\
\text { (1) CIE coordinates: }(0.32,0.25) \\
\text { at } 100 \mathrm{~K},(0.30,0.32) \text { at } 50 \mathrm{~K} \\
\text { (2) } \mathrm{CIE} \text { coordinates: }(0.31,0.25) \\
\text { at } 200 \mathrm{~K},(0.30,0.30) \text { at } 150 \mathrm{~K}\end{array}$ & $\begin{array}{l}{[133]} \\
(2017)\end{array}$ \\
\hline
\end{tabular}




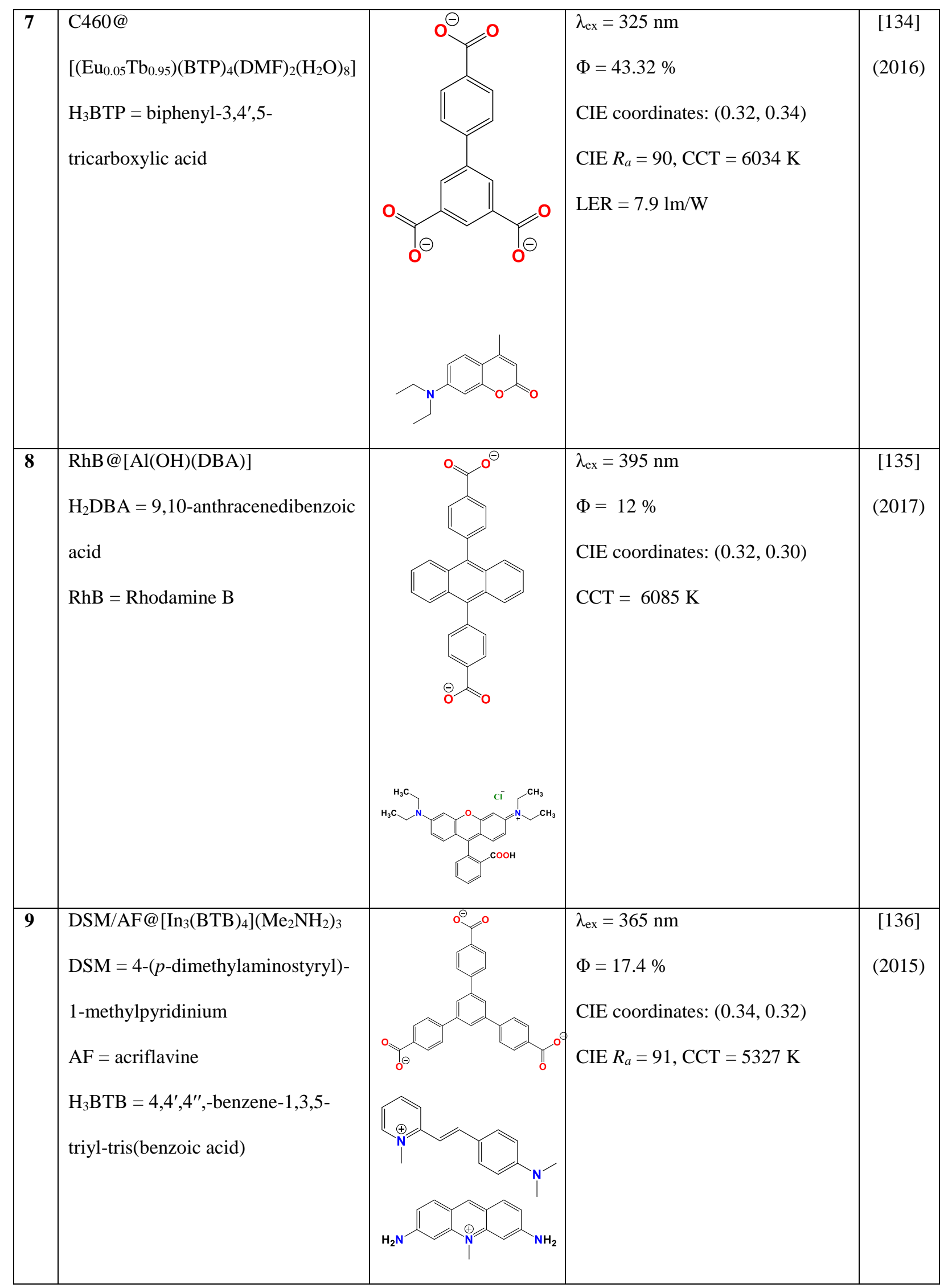




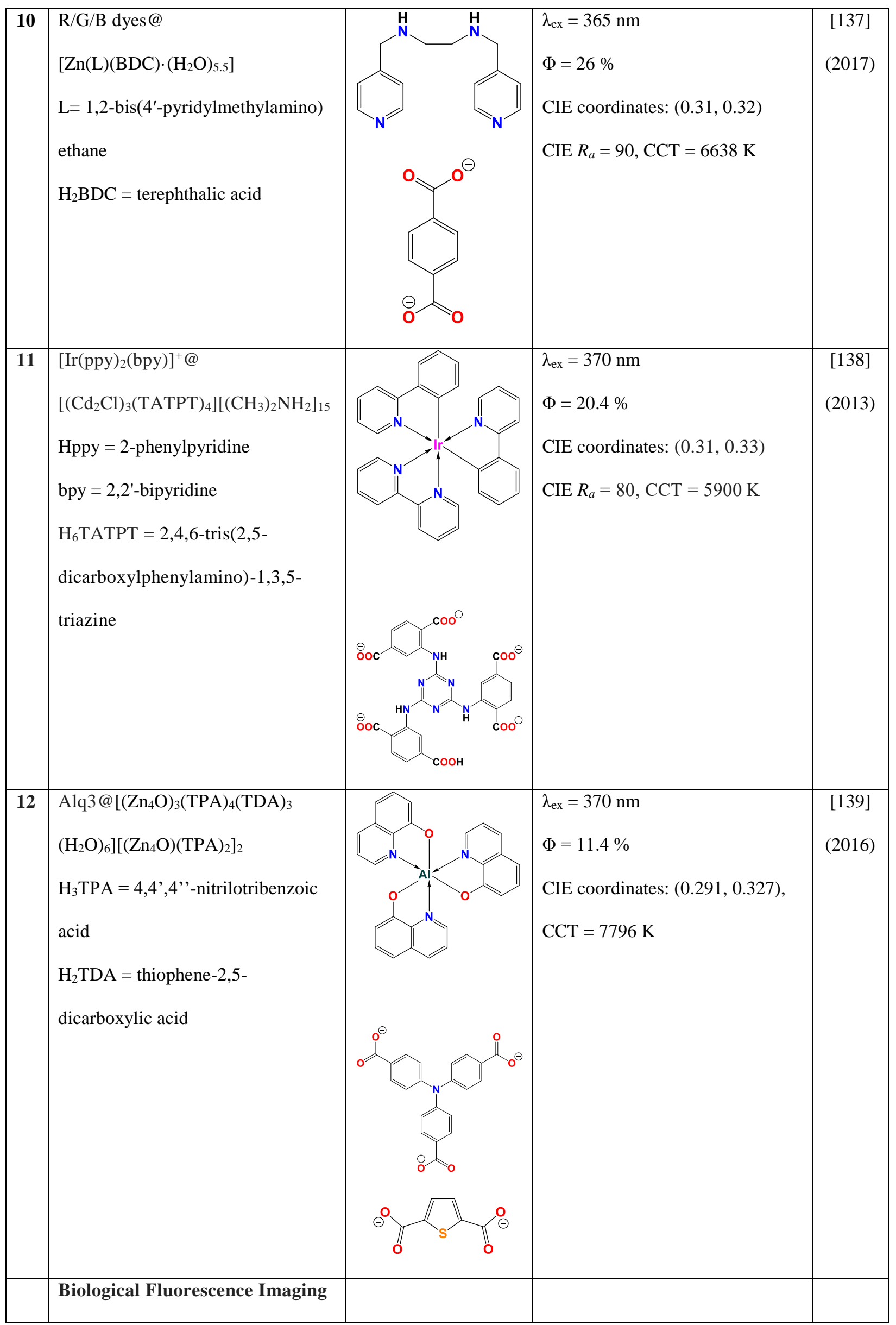




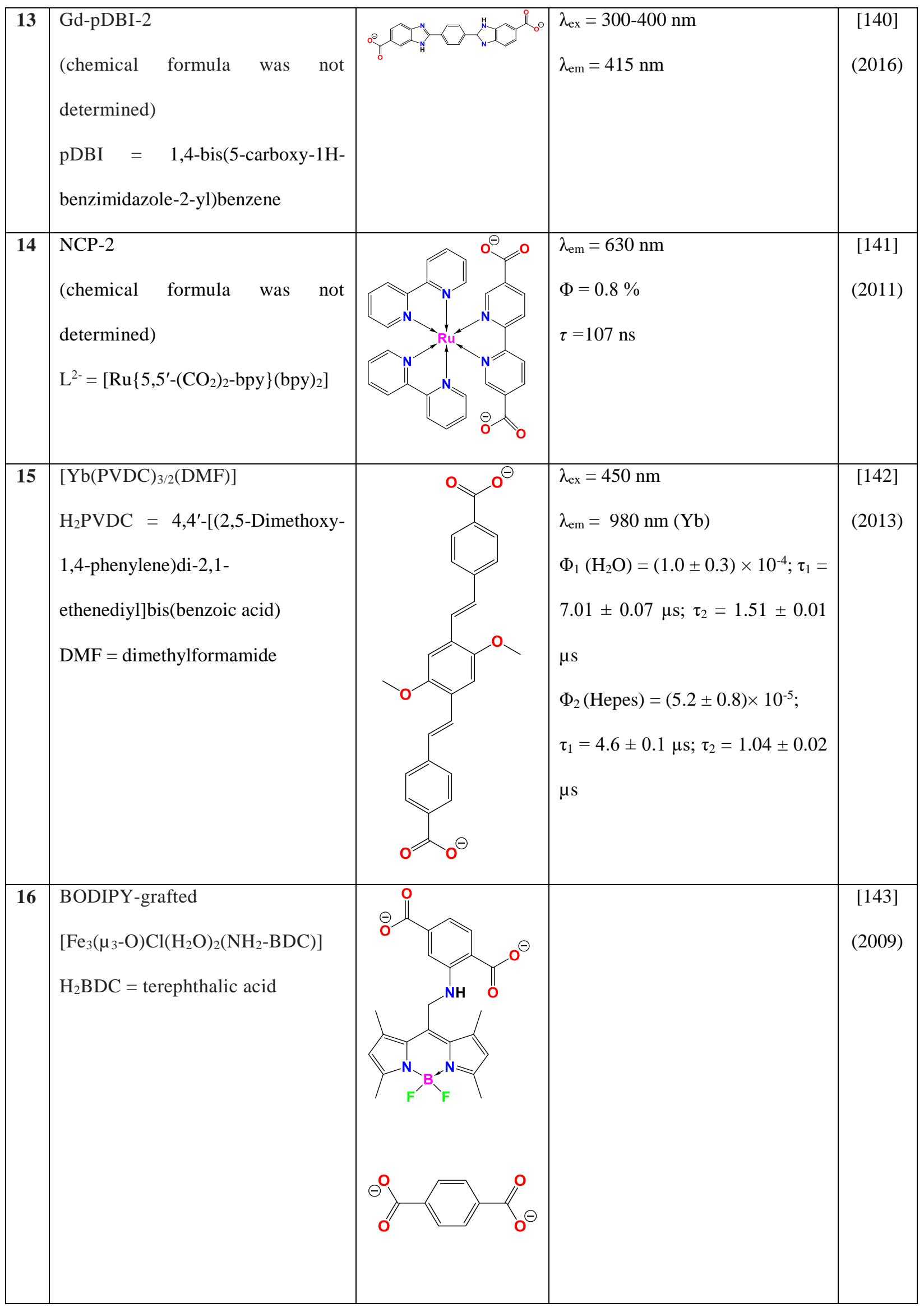




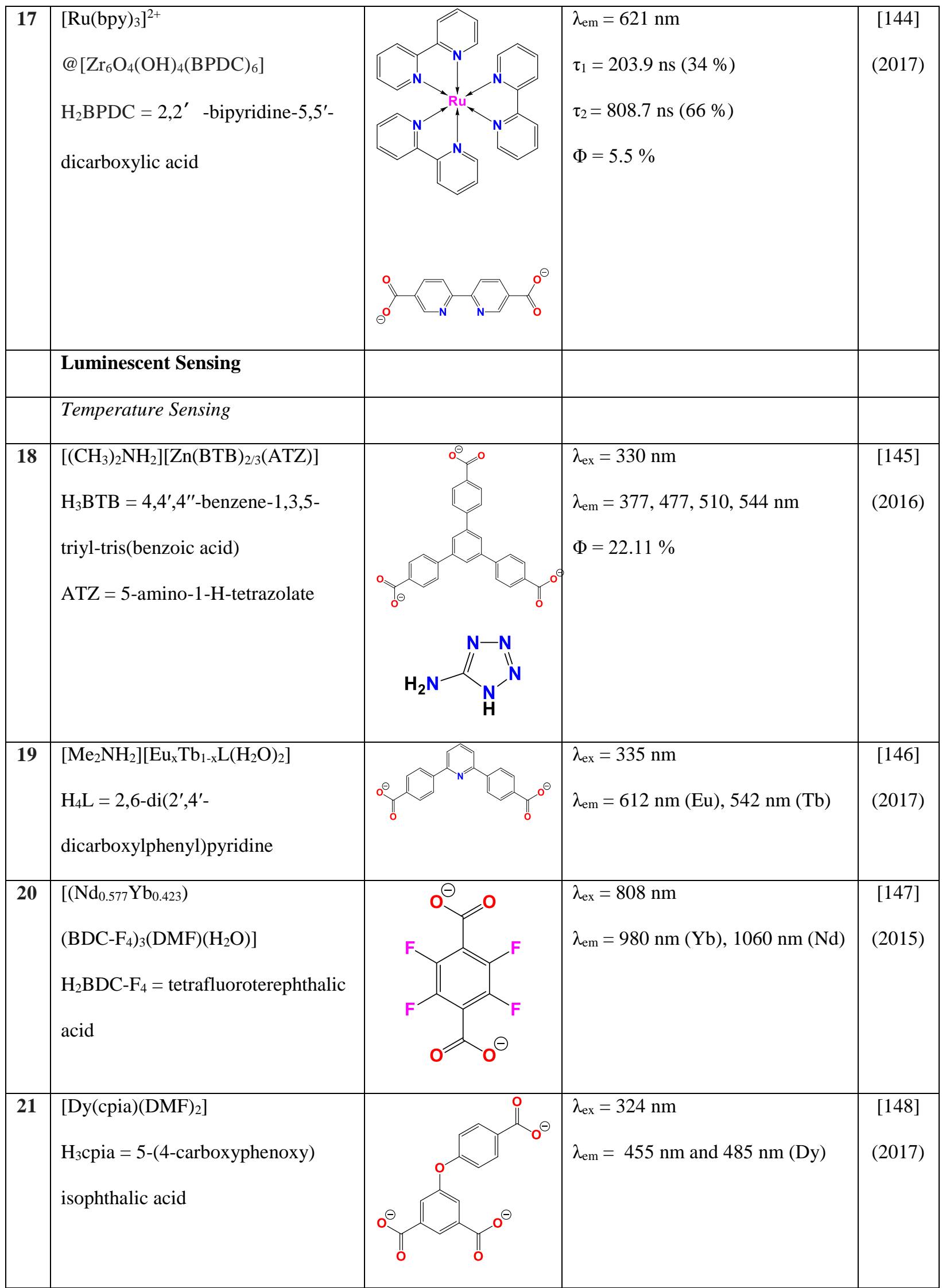




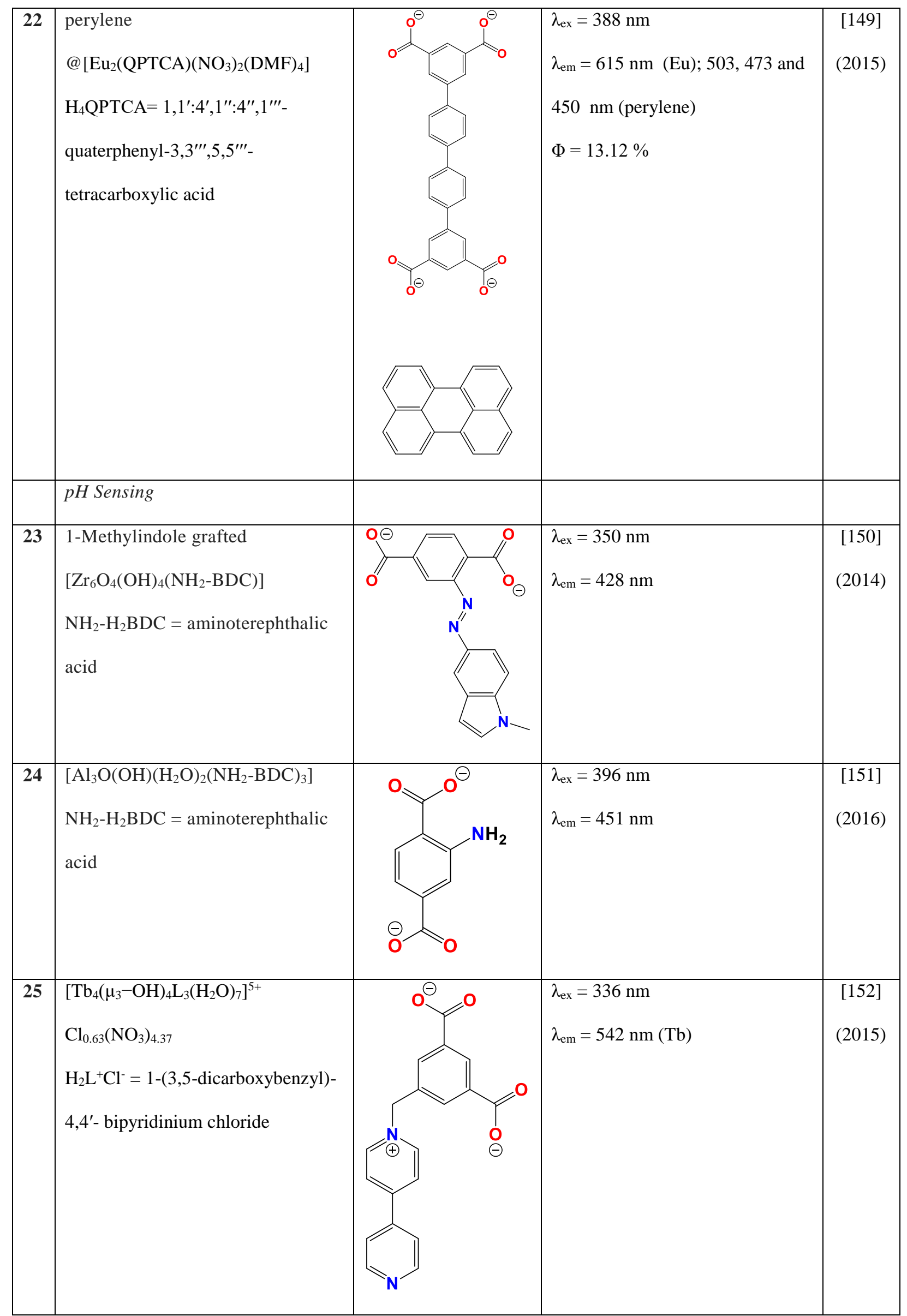




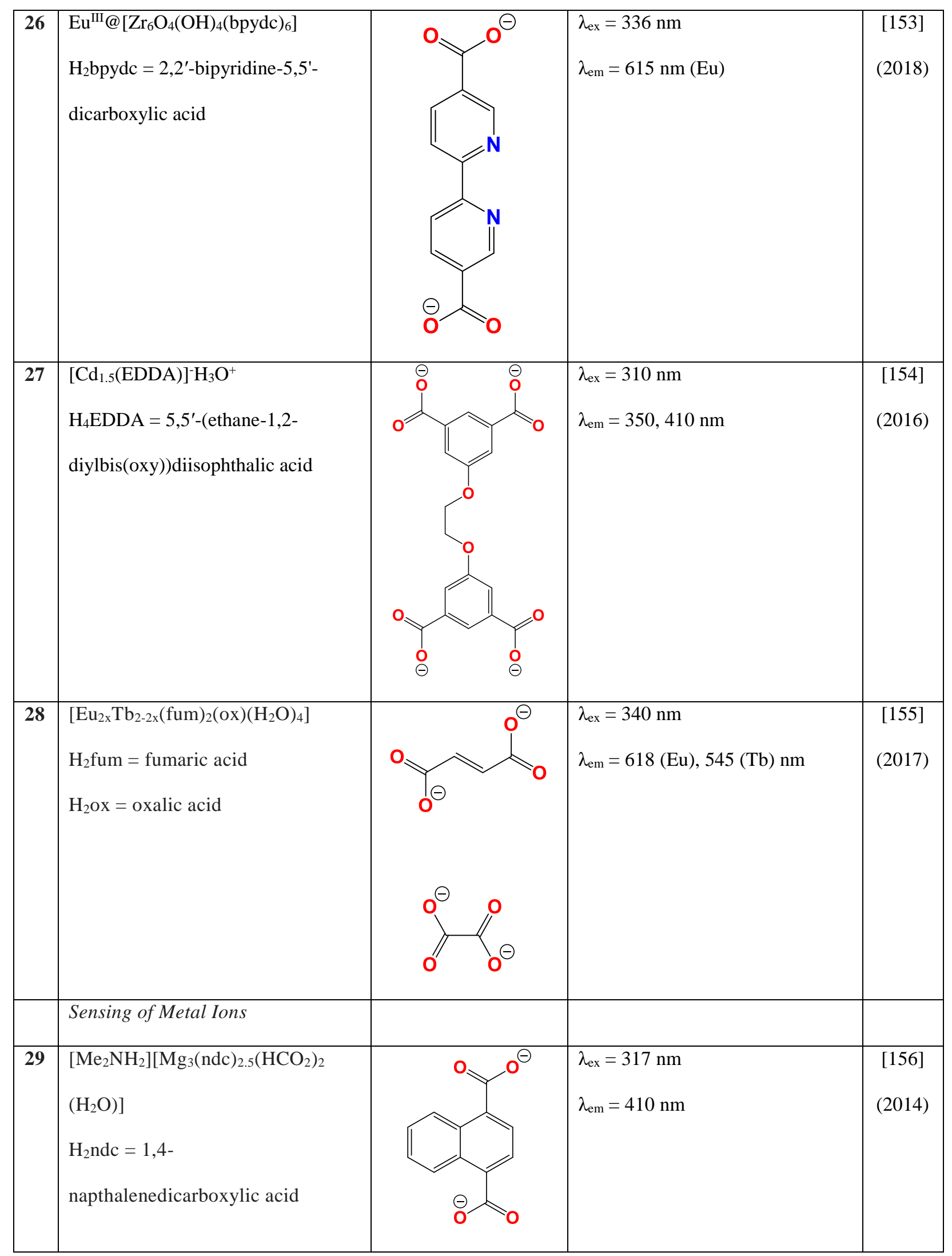




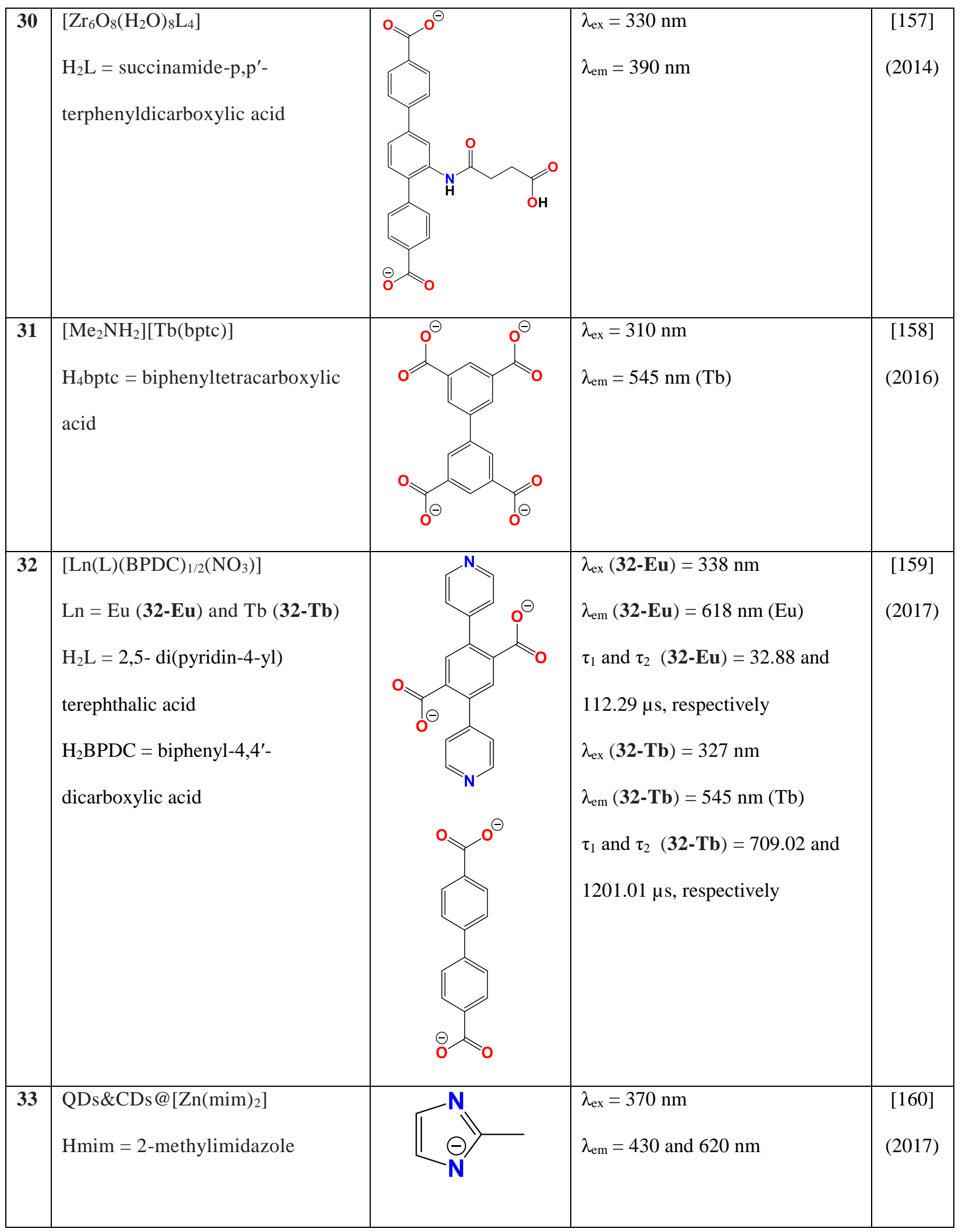




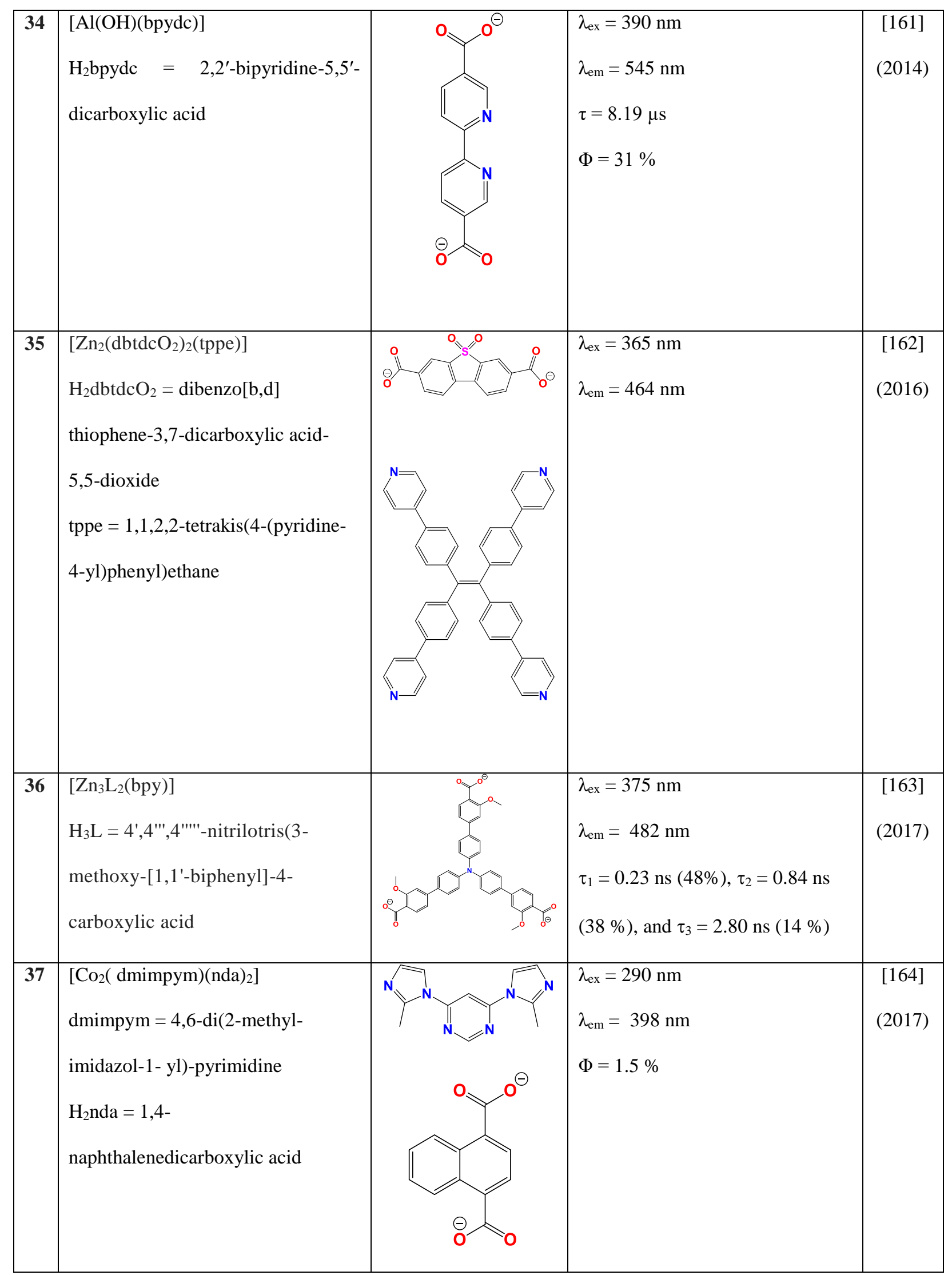




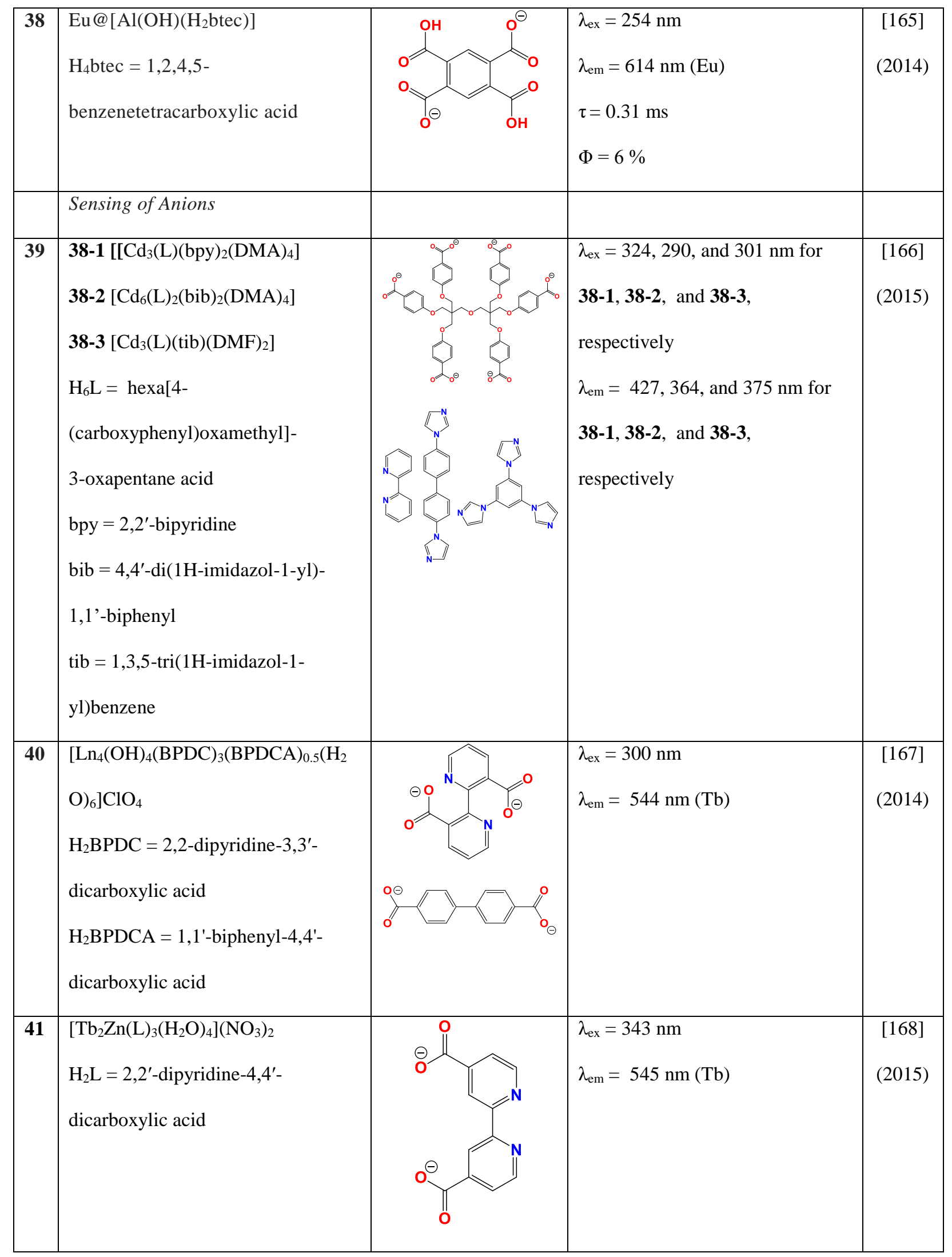




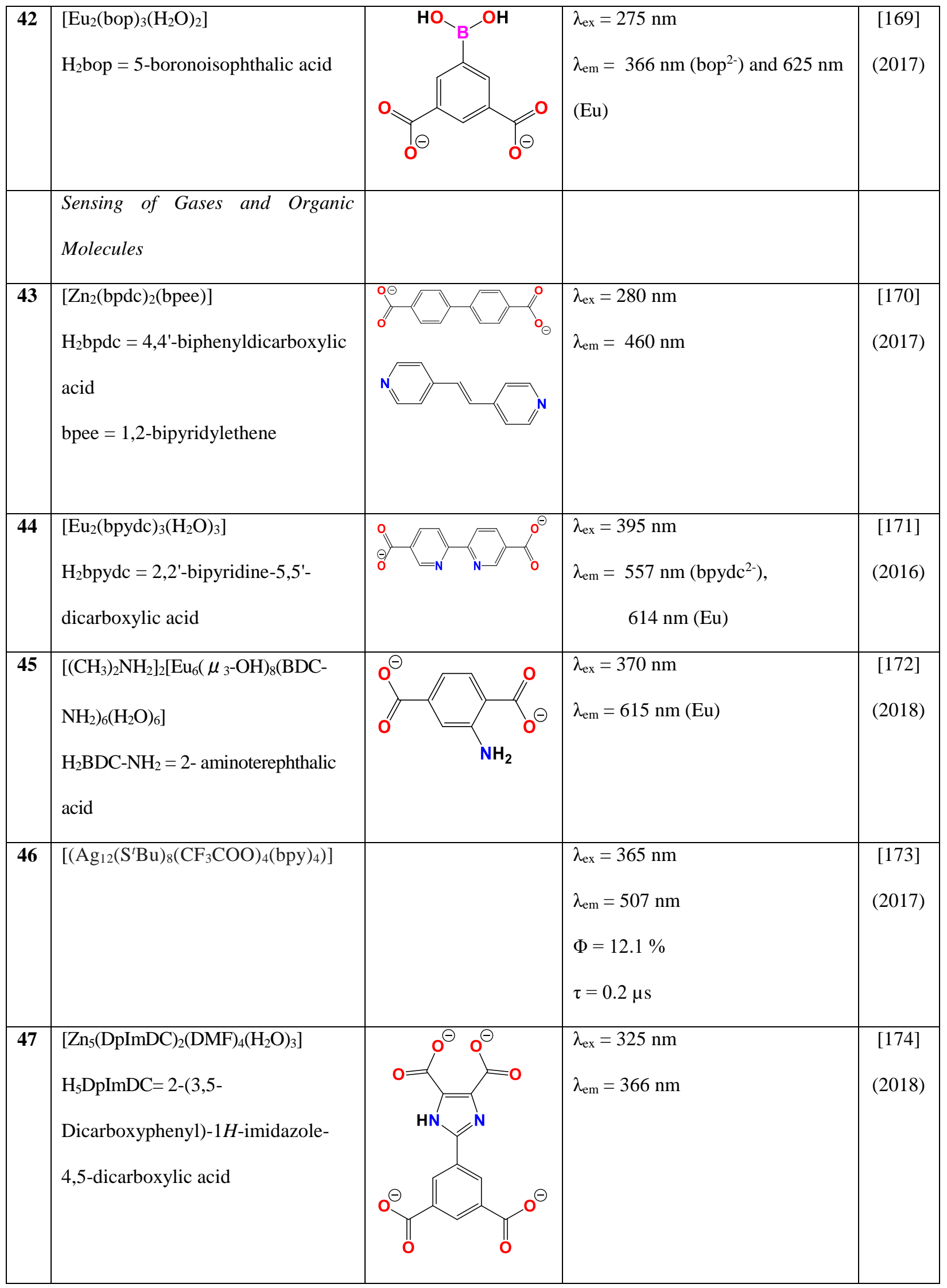




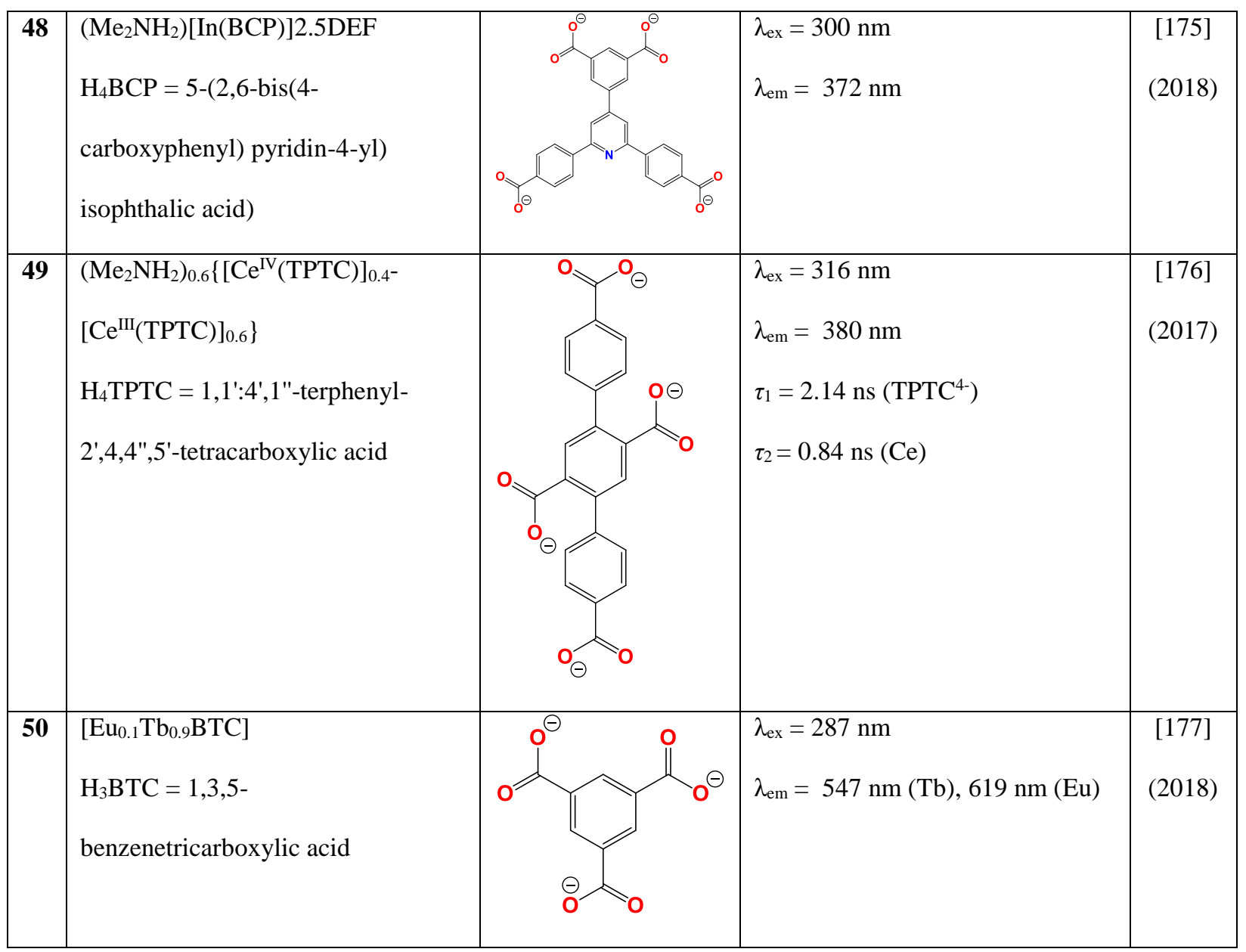

\subsubsection{White-light Emission}

\subsubsection{Fundamental principles}

White light is the type of light with the most universal applications, both indoors and outdoors. Nowadays, the majority of white-lighting devices are incandescent and fluorescent lamps. An incandescent bulb produces light by heating a tungsten filament to high temperature, which emits white light of less than $5 \%$ efficiency [178]. The remaining energy is converted into heat. A fluorescent lamp is normally a long narrow glass tube that is filled with a noble gas and low-pressure mercury gas. When a high voltage is applied to the electrodes at the ends of the tube, some electrons are accelerated, collide and transfer energy to the mercury atoms, whose electrons are promoted to an excited state. When these excited electrons return to the ground state, the transition produces a UV light of $254 \mathrm{~nm}$, which is 
further used to excite the phosphor powder coated on the inner surface of the glass tube and eventually produces a near white light. Although fluorescent lamps are more efficient than incandescent lamps for converting energy into useful light, their efficiencies are still quite low, of $\sim 20 \%$. In addition, fluorescent lamps contain mercury, which complicates their disposal. In recent years, white light emitting diodes (LEDs), which are typically made from a blue LED with part of its light converted to green and red by utilizing one or several phosphor compounds, have been developed. They have advantages over the traditional lamps such as small size, long lifetime, and high efficiency; however, one of their remaining disadvantages is the lack of high white light quality.

In general, a light source for lighting is evaluated based on three main criteria: the Commission internationale de l'éclairage (CIE) coordinates, the luminous efficacy of the radiation (LER), and the color quality. The CIE coordinates are calculated from the spectral power distribution of the light source using the CIE color-matching functions, and are used to determine the color of the light based on the chromaticity diagram, as shown in Figure 5. High-quality white-light illumination requires a source with CIE coordinates of (0.333; $0.333)$. 


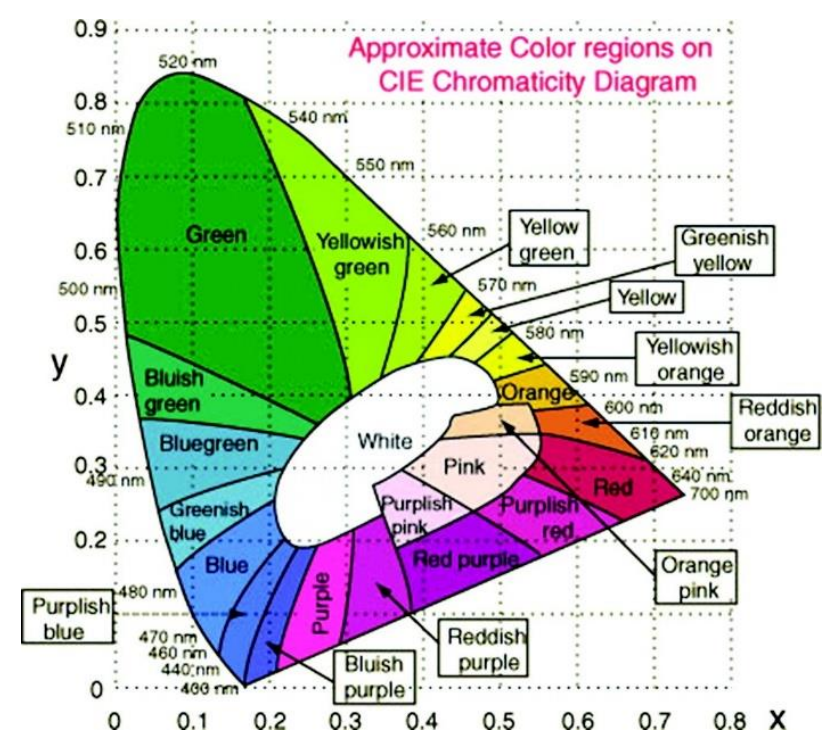

Figure 5. A typical CIE-1931 chromaticity diagram. The region of all perceptible colors is encompassed by a horseshoe-shaped curve contain the spectral colors. Reproduced with permission from reference [129].

LER $(\mathrm{lm} / \mathrm{W})$ is a parameter describing the brightness of the radiation perceived by the average human eye and can be obtained from the emission spectrum, as shown in equation 4,

$L E R=683 \frac{\int_{360}^{830} I_{\lambda} V_{\lambda} d \lambda}{\int_{360}^{830} I_{\lambda} d \lambda}$

where $I_{\lambda}$ and $V_{\lambda}$ are the radiometric power and the eye sensitivity curve at wavelength $\lambda$, respectively. The theoretical limit of LER $(683 \mathrm{~lm} / \mathrm{W})$ is obtained from a monochromatic green radiation at $555 \mathrm{~nm}$, which has the maximum spectral sensitivity of the human eye under daylight conditions. For white light, which primarily requires the combination of green, red, and blue lights, the LER value is lower than $683 \mathrm{~lm} / \mathrm{W}$. The LER of a typical incandescent, fluorescent, and LED bulb is of $\sim 12 \mathrm{~lm} / \mathrm{W}, \sim 80 \mathrm{~lm} / \mathrm{W}$, and $\sim 150 \mathrm{~lm} / \mathrm{W}$, respectively [179]. A good light source should have not only a maximum LER but also high color quality, which is characterized by the color rendering index (CRI). The concept of CRI is based on comparing the color of test objects to the colors of these objects illuminated by an infinite number of reference sources. At first, the spectrum of the test light source is 
compared to the one of a black body radiator allowing for determination of its correlated color temperature (CCT). For light sources with CCT above $5000 \mathrm{~K}$, i.e. cool light, a standard daylight spectrum of the same CCT is used, whereas for those with CCT below $5000 \mathrm{~K}$, i.e. warm light, the reference source is a black body radiator. The general color rendering index $R_{\mathrm{a}}$ is calculated as in equation 5 ,

$R_{a}=100-4.6 \times \frac{1}{n} \sum_{n} \Delta E_{i}$

where $n$ is the number of test objects, usually $n=8 ; \Delta E_{\mathrm{i}}$ is the difference between the color of the test object $i$ illuminated with the test and reference light source. The theoretical CIE $R_{\mathrm{a}}$ value is 100 , and would only be given to a source identical to standardized daylight or a black body. Normally, light sources with CIE $R_{\mathrm{a}}$ above 95 are regarded as giving high color rendering quality.

\subsubsection{White-light emitting MOFs}

White light can be obtained from materials that exhibit multiple-color emissions, e.g. a combination of red, green, and blue emissions with proper proportions between them can give white light. MOFs emitting white light can be designed by utilizing intra- and/or interligand charge transfer, ligand-to-metal charge transfer (LMCT), metal-to-ligand charge transfer (MLCT), doping with lanthanides, or by encapsulating luminescent organic dyes and metal complexes. An example of a ligand-based luminescent MOF emitting white light based on intra- and interligand charge transfers was reported by Li et al. [128]. The Cd MOF (1: entry 1 in Table 1$)$ was synthesized based on the ligand tzphtpy ${ }^{-}($Htzphtpy $=4$-(tetrazol-5yl)phenyl-2,2':6',2"-terpyridine), which coordinates to $\mathrm{Cd}^{\mathrm{II}}$ ions through one $\mathrm{N}$ of the tetrazole group and one $\mathrm{N}$ from the terpyridine group to generate a $1 \mathrm{D}$ pearl-necklace-like chain. The supramolecular 3D framework is formed through $\pi-\pi$ interactions between the adjacent $1 \mathrm{D}$ chains. Luminescence studies on $\mathbf{1}$ at room temperature revealed that the MOF 
displays two broad emissions centered at 454 and $567 \mathrm{~nm}$ when excited at $386 \mathrm{~nm}$, with their respective lifetimes of 8.26 and $9.89 \mathrm{~ns}$ typical for fluorescence. The intensities of both emissions are comparable when varying the excitation wavelengths between 286 and $386 \mathrm{~nm}$ suggesting that the dual emissions originate from different luminous centers. Density-of-state (DOS) calculation showed that $\mathrm{Cd}^{\mathrm{II}}$ ions make little or no contribution to the absorption, and the emissions of $\mathbf{1}$ stem from ligand-centered transitions. In addition, the emission spectrum of Htzphtpy in $\mathrm{MeOH}$ shows only one emission band centered at $422 \mathrm{~nm}$ suggesting that the lower emission band at $567 \mathrm{~nm}$ in the solid state originates from interligand charge transfer associated with the $\pi-\pi$ interactions between the 1D chains. White-light emission of 1 was obtained when the MOF was excited at $326 \mathrm{~nm}$, (Figure 6) with CIE coordinates of (0.33, 0.36), a CIE $\mathrm{R}_{\mathrm{a}}$ of 77 , a favorable CCT magnitude of $5328 \mathrm{~K}$, and a relatively low quantum yield of $2.3 \%$.

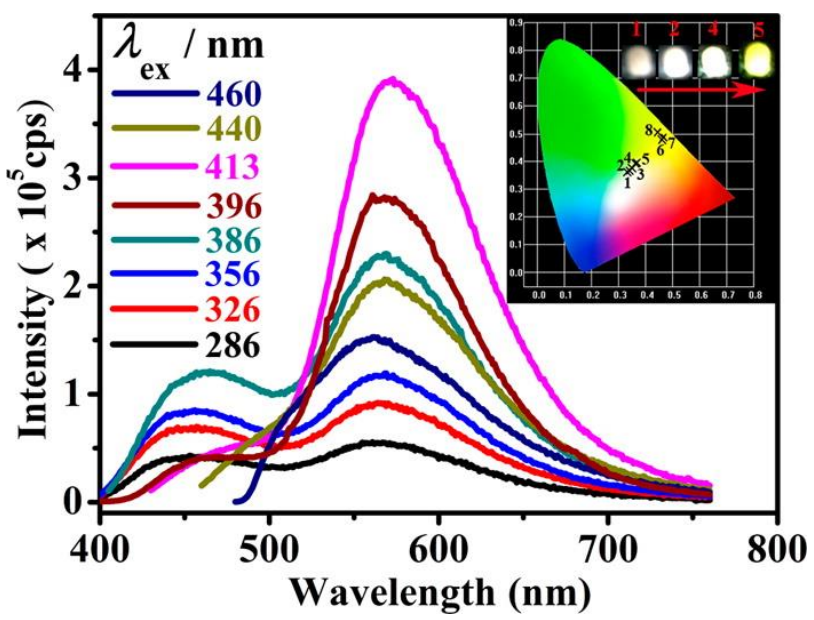

Figure 6. Emission spectra of the Cd MOF (1). Inset: the CIE-1931 chromaticity diagram and optical images of the powdered samples of $\mathbf{1}$. Reproduced with permission from reference [128].

He et al. reported a $\mathrm{Pb} \mathrm{MOF}$ (2) that emits white light based on both ligand-centered and LMCT emissions [129]. 2 was obtained from a solvothermal synthesis using 2,5-bis((2hydroxylpropyl)thio)-1,4-benzene dicarboxylic acid. The structure builds on a rod-packing 
motif with the $\mathrm{Pb}$-carboxylate rods linked by the aromatic moieties into a 3D framework. Under UV radiation at room temperature, the solid sample of 2 exhibits a bright, white luminescence with a high energy band maxima at $459 \mathrm{~nm}$ and a weaker low energy band tailing into the red region beyond $600 \mathrm{~nm}$, which was attributed to the LMCT between the aromatic $\pi$ systems and the $\mathrm{p}$ orbitals of the $\mathrm{Pb}^{\mathrm{II}}$ centers. The quantum efficiency of $\mathbf{2}$ is rather low, of $2-3 \%$; the CIE coordinates are $(0.27,0.30),(0.25,0.29)$, and $(0.24,0.28)$ with excitations at 300, 350, and $400 \mathrm{~nm}$, respectively. These coordinates are all falling within the white gamut of the CIE-1931 color space chromaticity diagram.

Wang et al. utilized 4-cyanobenzoate ligand to construct a 2D Ag MOF (3) that emits white light based on the combination of intraligand and MLCT emissions [130]. The 2D frameworks stack in parallel without interpenetration to generate the 3D structure, in which Ag $\cdots$ Ag interactions and $\pi-\pi$ stacking interactions are present among the layers. The solidstate sample of 3 displays broad emissions centered at $\sim 427$ and $\sim 566 \mathrm{~nm}$ when excited under 355- and 330-nm UV radiation. While the high energy emission has a lifetime of 0.87 ns typical for fluorescence and was assigned to the $\pi-\pi^{*}$ intraligand transition, the low energy emission exhibits a long lifetime of $2.60 \mathrm{~ms}$ suggesting a phosphorescence characteristic. This emission was explained by the presence of a MLCT transition between the Ag $4 \mathrm{~d}$ electrons and the $\pi^{*}$ orbitals of the ligand. When adjusting the excitation light to $349-350 \mathrm{~nm}$, the emission peaks at 427 and $566 \mathrm{~nm}$ are comparable in intensity, which results in a whitelight emission with a quantum yield of $10.86 \%$. The CIE coordinates are $(0.31,0.33)$ and $(0.33,0.34)$ for the excitation at 350 and $349 \mathrm{~nm}$, respectively.

Doping with lanthanides emitting in visible regions is a common and efficient method to tune luminescence emissions to obtain white light. Sava et al. reported an In MOF (4, also known 
SMOF-1) synthesized from a solvothermal reaction utilizing benzene-1,3,5-triyl trisbenzoate $\left(\mathrm{BTB}^{3-}\right)$ and oxalate as ligands [131]. Luminescence studies revealed that 4 emits a ligand-based emission centered at $390 \mathrm{~nm}$ after being excited at $330 \mathrm{~nm}$ and a broad-band emission when the material is excited between 350 and $380 \mathrm{~nm}$, which was attributed to the $\mathrm{BTB}^{3-} \rightarrow \mathrm{In}^{\mathrm{III}}$ LMCT. This results in direct white-light emission with CIE $\mathrm{R}_{\mathrm{a}}$ values within the range of 81-85, but with very high CCTs (21642-33290 K). To improve the color properties, the authors doped 4 with Eu ${ }^{\text {III }}$ in different amounts reaching $10 \%$ of the total mol of $\operatorname{In}^{\mathrm{III}}$. The $10 \%$ Eu-doped material emits high-quality white light with CIE $R_{a} \sim 90$ and CCT $\sim 3200 \mathrm{~K}$. The CIE coordinates vary with excitation wavelengths; when the sample is excited at 350, 360,380 , and $394 \mathrm{~nm}$, the coordinates are $(0.369,0.301),(0.309,0.298),(0.285,0.309)$, and $(0.304,0.343)$, respectively (Figure 7$)$. A modest quantum yield of $4.3 \%$ was obtained when the sample was excited at $330 \mathrm{~nm}$; however, the material exhibits a fairly long lifetime of 600 $\mu$ s due to the long-lifetime characteristic of the $\mathrm{Eu}^{\mathrm{III}}$ ion. It is worth noting that doping ligandbased luminescent MOFs with more than one lanthanide ion for obtaining white light emission is also possible. An example was the work by Mondal et al., who reported the codoping of both $\mathrm{Eu}^{\mathrm{III}}$ and $\mathrm{Tb}^{\mathrm{III}}$ into a Zn-based zeolitic imidazolate framework (ZIF) [180], which exhibits white emission. However, the quantum yield was not reported for this material. 


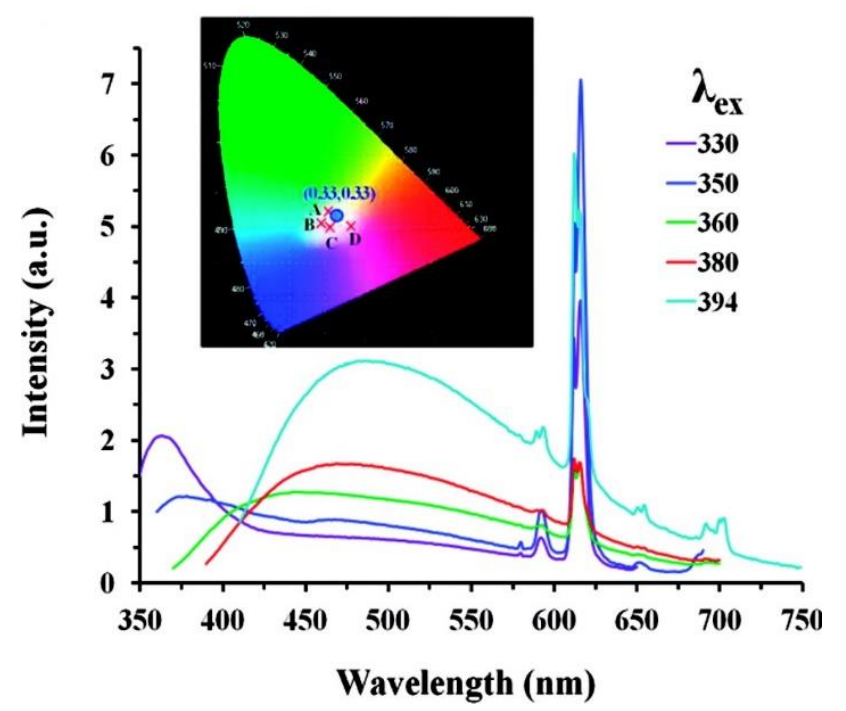

Figure 7. Emission spectra of $10 \%$ Eu-doped 4 at different excitation wavelengths. Inset: CIE chromaticity diagram. Reproduced with permission from reference [131].

Park et al. designed a mixed lanthanide MOF (5) incorporated with a small amount of the blue-emitting 2-(pyrene-1-carboxamido)terephthalate ligand $\left(\mathrm{L}^{2-}\right)$ to obtain white light emission [132]. The authors presented a nice and systematic procedure to find the optimal amount of each component of the MOF, i.e. $\mathrm{Eu}^{\mathrm{III}}, \mathrm{Tb}^{\mathrm{III}}$, and $\mathrm{L}^{2-}$, responsible for the red, green, and blue color emissions. To find the optimized amount of $\mathrm{L}^{2-}$ for effective blue emission, a series of Gd-analog MOFs $\left[\mathrm{Gd}_{3}(\mathrm{BDC})_{4.5-\mathrm{x}}(\mathrm{L})_{\mathrm{x}}(\mathrm{S})_{\mathrm{a}}\right]$ with various $\mathrm{BDC}^{2-}: \mathrm{L}^{2-}$ ratios were prepared. The ratio of $0.975: 0.025$ was found to display the highest blue emission. Similarly, to find the optimized amount of the lanthanide ions, a series of $\left[\mathrm{Eu}_{3-\mathrm{y}} \mathrm{Tb}_{\mathrm{y}}(\mathrm{BDC})_{4.5}(\mathrm{~S})_{\mathrm{a}}\right]$ MOFs were investigated by varying the ratios of $\mathrm{Eu}^{\mathrm{III}}: \mathrm{Tb}^{\mathrm{III}}$. The ratio of $0.1: 0.9$ gave the ideal yellow emission, which in theory can combine with the blue emission from $\mathrm{L}^{2-}$ to give white-light emission. The excitation of the MOF $\left[\mathrm{Eu}_{0.3} \mathrm{~Tb}_{2.7}(\mathrm{BDC})_{4.3875}(\mathrm{~L})_{0.1125}(\mathrm{~S})_{\mathrm{a}}\right]$ at different wavelengths in the range of $300-310 \mathrm{~nm}$ led to emissions with CIE coordinates changed from $(0.341,0.400)$ to $(0.223,0.223)$. The 
coordinates of $(0.302,0.342)$, which are close to those of pure white light $(0.333,0.333)$, were obtained with the excitation at $304 \mathrm{~nm}$.

Gai et al. demonstrated white-light emission from a three-lanthanide MOF (6) prepared from p-terphenyl-2.2",4,4"-tetracarboxylic acid $\left(\mathrm{H}_{4} \mathrm{~L}\right)$ and a mixture of sulfate salts of Eu ${ }^{\mathrm{III}}$, Dy ${ }^{\mathrm{III}}$, and $\mathrm{Tb}^{\mathrm{III}}$ [133]. By varying the ratio of the lanthanide ions, the mixed lanthanide MOFs, $\left[\mathrm{Eu}_{\mathrm{x}} \mathrm{Tb}_{\mathrm{y}} \mathrm{Dy}_{1-\mathrm{x}-\mathrm{y}}(\mathrm{HL})\left(\mathrm{H}_{2} \mathrm{O}\right)(\mathrm{DEF})\right]$ show emission peaks with intensities changing as the $\mathrm{Eu}^{\mathrm{III}}$, $\mathrm{Tb}^{\mathrm{III}}$, and $\mathrm{Dy}{ }^{\mathrm{III}}$ concentrations changed. The main emissions are from the emissive states of $\mathrm{Eu}^{\mathrm{III}}$ and $\mathrm{Tb}^{\mathrm{III}}$ ions together with a relatively weak emission band around $380-450 \mathrm{~nm}$ stemming from the HL ligand. The optimized MOF $\left[\mathrm{Eu}_{0.0667} \mathrm{~Tb}_{0.0667} \mathrm{Dy}_{0.8666}(\mathrm{HL})\left(\mathrm{H}_{2} \mathrm{O}\right)(\mathrm{DEF})\right]$ does not exhibit white-light emission at room temperature; however, when the temperature is decreased to a value between 100 and $50 \mathrm{~K}$, the emission possesses CIE coordinates of $(0.32$, $0.25)$ at $100 \mathrm{~K}$ and $(0.30,0.32)$ at $50 \mathrm{~K}$ that are very close to those of the pure white light (0.33, 0.33). Similarly, the MOF $\left[\mathrm{Eu}_{0.0666} \mathrm{~Tb}_{0.4667} \mathrm{Dy}_{0.4667}(\mathrm{HL})\left(\mathrm{H}_{2} \mathrm{O}\right)(\mathrm{DEF})\right]$ also exhibits white light emission when the temperature varies between $200 \mathrm{~K}$ and $150 \mathrm{~K}$, with the CIE coordinates being $(0.31,0.25)$ at $200 \mathrm{~K}$ and $(0.30,0.30)$ at $150 \mathrm{~K}$.

The encapsulation of organic dyes in lanthanide-based MOFs is another method for tuning the emission in order to obtain white light. Song et al. demonstrated this strategy in a $\mathrm{Eu}^{\mathrm{III}} / \mathrm{Tb}^{\mathrm{III}}$-mixed MOF (7) that encapsulates the blue-light emitting dye coumarin 460 (C460) into the pores by the in situ synthesis [134]. The optimized ratio of $\mathrm{Eu}^{\mathrm{III}}$ and $\mathrm{Tb}^{\mathrm{III}}$ in the $\left[\left(\mathrm{Eu}_{\mathrm{x}} \mathrm{Tb}_{1-\mathrm{x}}\right)(\mathrm{BTP})_{4}(\mathrm{DMF})_{2}\left(\mathrm{H}_{2} \mathrm{O}\right)_{8}\right] \mathrm{MOF}$ was found to be $0.05: 0.95$ to give a yellow emission when excited under UV light. The composite 7 with different contents of C460 was subjected to emission measurements, which showed that the broad emission band of C460 around 460 $\mathrm{nm}$ was enhanced with the increase in dye content, while the intensity of the characteristic 
lanthanide ion emissions was reduced. The composite of $1.02 \mathrm{wt} \%$ of the coumarin dye exhibits an emission with the CIE coordinates of $(0.32,0.34)$, which is very close to those of ideal white light $(0.33,0.33)$. Moreover, 7 possesses a quantum yield of $43.42 \%$, a CIE $R_{a}$ value of 90 , and a CCT of $6034 \mathrm{~K}$. The authors went further to fabricate the white-lightemitting diode (WLED) by coating the composite 7 on a commercial $365 \mathrm{~nm}$ UV-LED chip (Figure 8). The prototype WLED emitted bright white light with a LER value of $7.9 \mathrm{~lm} / \mathrm{W}$ when a $3 \mathrm{~V}$ bias was applied.
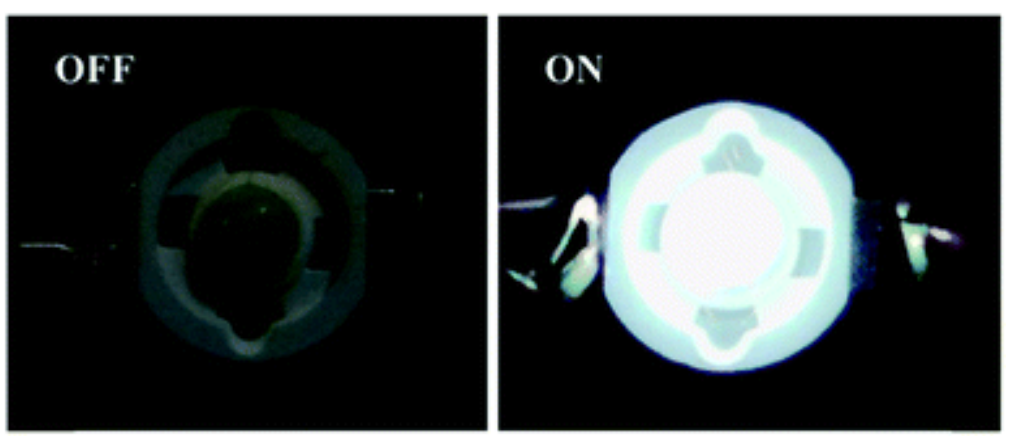

Figure 8. Photographs of a prototype WLED fabricated with 7 when it was turned on (right) and off (left). Reproduced with permission from reference [134].

For MOFs formed from metal ions other than lanthanides, utilizing luminescent emissions from ligands and encapsulated organic dyes or luminescent transition metal complexes is also a feasible approach to obtain white-light emission. Recently, Wang et al. reported a dyeencapsulated MOF (8) to build a warm-white-light emitting diode for fast white-light communication [135]. 8 was synthesized based on the employment of 9,10anthracenedibenzoic acid $\left(\mathrm{H}_{2} \mathrm{DBA}\right)$ and hence the MOF itself (Al-DBA) exhibits a blue emission originated from the $\mathrm{DBA}^{2-}$ ligand, with a maximum wavelength at $468 \mathrm{~nm}$. Encapsulation of $\mathrm{RhB}$ leads to a material that exhibits both $\mathrm{RhB}$ yellow and blue $\mathrm{DBA}^{2-}$ emissions, with the ratio between the two depending on the dye loading level. At 0.019 mol 
$\%$ loading, warm-white emission was achieved with the CIE coordinates of $(0.32,0.30)$, a $\mathrm{CCT}$ of $6085 \mathrm{~K}$, and a quantum yield of $12 \%$. Since the RhB emission decays back to the ground state with a $\mathrm{t}_{1 / 2}$ of $5.4 \mathrm{~ns}$, which is much faster than that of the yellow $\mathrm{Y}_{3} \mathrm{Al}_{5} \mathrm{O}_{12}: \mathrm{Ce}^{3+}$ (YAG-Ce) phosphor, $\mathbf{8}$ is ideal for use in white-light communication.

Similarly, Cui et al. presented a two-dye-encapsulated MOF (9) for warm-white LED with high CIE $R_{a}$ value [136]. The MOF itself (called ZJU-28), formed by using 4,4',4"'-benzene1,3,5-triyl-trisbenzoate $\left(\mathrm{BTB}^{3-}\right)$, exhibits a blue emission centered at $415 \mathrm{~nm}$ that was attributed to the emission derived from the ligand. Encapsulation of the DSM and the AF dyes led to composites that display red and green colors, respectively. Therefore, the authors utilized both dyes for tuning the emission of the composite. The optimal white-light emission was realized when the concentration of DSM and AF was adjusted to $0.02 \mathrm{wt} \%$ and 0.06 wt $\%$, respectively. High-quality light was achieved; the CIE coordinates of $(0.34,0.32)$, a CCT value of $5327 \mathrm{~K}$, a CIE $R_{a}$ value of 91 , and a quantum yield of $17.4 \%$ were reported. A WLED device was fabricated by simply coating the composite as phosphor at the curved surface of the UV-LED chip. The resultant device exhibits bright white color when the chip was connected to the electric source of $3.8 \mathrm{~V}$.

The encapsulation of up to three organic dyes within a MOF (10) for white-light emission was also reported [137]. 10 (also called HSB-W1) is a Zn-based MOF and comprises two ligands: $\mathrm{BDC}^{2-}$ and 1,2-bis(4'-pyridylmethylamino)ethane (L). The MOF is featured by its high accessible void of $55.4 \%$ and easy encapsulation of linear organic dyes. When the three dyes including DCM (0.03 wt\%), C6a (0.02 wt \%), and CBS-127 $0.02 \mathrm{wt} \%$ were combined and inserted into the MOF, and excited at $365 \mathrm{~nm}$, the resulting material displayed white light, with CIE coordinates of $(0.31,0.32)$, CIE $R_{a}$ of 90 , a CCT value of $6638 \mathrm{~K}$, and a 
quantum yield of $26.0 \%$. Interestingly, the method is quite versatile as changing dyes such as DSM for DCM, C6 for C6a, or KSN for CBS-127 still leads to materials that exhibit whitelight emission. A WLED was also fabricated by simply coating the solid of dye-doped $\mathbf{1 0}$ on the surface of a commercially available ultraviolet LED chip ( $3 \mathrm{~mm}, 365 \mathrm{~nm})$. The resultant WLED device emitted bright white light at a voltage of $3.8 \mathrm{~V}$.

Departing from the organic dye encapsulation, Sun et al. reported the white-light emission from a Cd MOF (11) that encapsulates an iridium polypyridine complex [138]. 11 was synthesized using the ligand 2,4,6-tris(2,5-dicarboxylphenylamino)-1,3,5-triazine (TATPT ${ }^{6-}$ ) and it emits a blue emission at 490nm. The formed MOF, with the formula of $\left[\left(\mathrm{CH}_{3}\right)_{2} \mathrm{NH}_{2}\right]_{15}\left[\left(\mathrm{Cd}_{2} \mathrm{Cl}\right)_{3}(\mathrm{TATPT})_{4}\right]$, contains ammonium counter-cations that can be exchanged with positively-charged metal complexes. The author chose to encapsulate an IrII complex, $\left[\operatorname{Ir}(\mathrm{ppy})_{2}(\mathrm{bpy})\right]^{+}$, due to its strong yellow emission originating from its characteristic ${ }^{3}$ MLCT. The composite samples maintain their crystallinity with the concentrations of encapsulated $\mathrm{Ir}^{\mathrm{III}}$ complex reaching $8.8 \mathrm{wt} \%$ with respect to $\mathrm{Cd}^{\mathrm{II}}$. For the sample of $3.5 \mathrm{wt} \%$ encapsulated $\mathrm{Ir}^{\mathrm{III}}$ complex, the emission obtained at an excitation wavelength of $370 \mathrm{~nm}$ has the CIE coordinates of $(0.31,0.33)$. A CIE $R_{a}$ value of 80 , a CCT value of $5900 \mathrm{~K}$, and a quantum yield of $20.4 \%$ were also obtained for this sample. WLED assemblies using the composite $\mathbf{1 1}$ were fabricated by coating the sample either on an UVLED or an InGaAsN chip, which proves the potential use of this composite for practical lightning applications.

Xie et al. utilized the same approach as for $\mathbf{1 1}$ but using a yellow-emission main-metal complex, Alq3 (tris(8-hydroxyquinoline)aluminium), as the encapsulant since it is much cheaper than the IrIII complex [139]. The blue-emitting MOF (called NENU-521) was 
synthesized using 4,4',4"-nitrilotribenzoate $\left(\mathrm{TPA}^{3-}\right)$ and thiophene-2,5-dicarboxylate (TDA $\left.{ }^{2-}\right)$ as the ligands. Similar to TATPT ${ }^{6-}$ mentioned above, the TPA $^{3-}$ ligand also emits blue light at 450 nm ensuring the blue emission of the MOF. The composite 12 (Alq3@NENU-521) with different concentrations of Alq3 exhibits tunable fluorescence emission from blue to green-yellow. At the optimal concentration of $4.14 \mathrm{wt} \%$, the emission possesses CIE coordinates of $(0.291,0.327)$, a CCT value of $7796 \mathrm{~K}$, and a quantum yield of $11.4 \%$. WLED assemblies were fabricated by coating this material over ultraviolet LEDs. White light was observed at a voltage of $3.8 \mathrm{~V}$; more interestingly, the WLED can maintain illuminating bright white light when continuously turned on for one month.

In all examples mentioned above, the white light emitter is based on a single MOF, which is chemically modified by doping and encapsulating components that exhibit commensurate colors of emission. Fang et al., however, utilized a simple mechanical mixing approach in which two MOFs were employed to form a two-component white light emitting material [181]. The blue phosphor $1 \mathrm{D}-\mathrm{Cu}_{2} \mathrm{I}_{2}(\mathrm{tpp})_{2}(\mathrm{bpp})$ (tpp = triphenylphosphine, bpp = 1,3-bis(4pyridyl)propane) was blended with either the yellow- or orange-emitting $\mathrm{Cu}_{4} \mathrm{I}_{4}$-based $2 \mathrm{D}$ and 3D compounds to produce composites that emit white emission with very high quantum yields, ranging from $70-82 \%$. These phosphors could also be deposited on a thin resin film, which was then coated onto the inner surface of a UV lamp to make a prototype lighting device.

In summary, a large number of MOF-based white light emitting materials have been reported in recent years. Compared to the commercially available WLEDs, which display low quality of white light (CIE $R a$ usually $<75)$, the MOF-based compounds often have quite higher CIE $R a$ values. However, the white light emitted from the MOF-based compounds 
is still limited to cool light $(\mathrm{CCT}>5000 \mathrm{~K})$, which is not ideal for indoor illumination. In addition, most studies do not investigate the luminous efficacy of the prototype MOF-based WLEDs, which is an important parameter for the practical applications of these WLEDs. Nevertheless, we believe that MOF-based WLEDs are still in their infancy, and with further development they might have the potential to replace the current commercially available WLEDs in the future.

\subsubsection{Biological Fluorescence Imaging}

\subsubsection{Fundamental principles}

Biological fluorescence imaging is a qualitative sensing technique that utilizes fluorescence microscopes to visualize biological objects such as cells and tissues. These objects can be imaged by utilizing the autofluorescence exhibited by themselves, which occurs due to the presence of aromatic compounds such as NADPH, collagen, riboflavin, or amino acids, or by tagging with exogenous fluorescent probes. A suitable probe for biological fluorescence imaging should be conveniently excitable and detectable, and should exhibit high luminescence intensity. Cultured cells and tissues require physiological environment, e.g. mammal cells need to be preserved in a buffer solution at $37^{\circ} \mathrm{C}$ and $\mathrm{pH}=7.4$; therefore, fluorescent probes used for imaging of these cells and tissues need to be stable in such conditions. Additional considerations include the possibility to deliver the probes into cells and their potential toxicity, e.g. for imaging of live cells, the probes must maintain the physiological and structural integrity of the cell; in other words, they must be non-toxic.

Fixed-cell imaging normally involves five steps: i) fixing and permeabilizing the cells of interest, ii) labelling, iii) detecting, iv) protecting, and v) imaging. The first step is used to protect and stabilized the cell structures and is often performed by utilizing a fixative agent 
such as formaldehyde to lock the cell, followed by using detergents to remove cellular membrane lipids to allow large fluorescent probes to get inside the cell. Labelling cells with the fluorescent probes allows for detecting the fluorescence signals in cells and separates these signals from the autofluorescent background. In some cases, when the fluorescent probes are prone to photobleaching leading to fast decreasing of fluorescence signals, antifade reagents can be used to protect the photostability of these probes. The final step of imaging involves the use of a fluorescence microscope to capture the images of the cells. The most commonly used fluorescence microscopes are the epifluorescence and confocal microscopes, with the latter being more advanced due to the employment of point laser illumination and a pinhole in front of the detector to block out-of-focus photons; therefore, their optical resolution and contrast is significantly increased compared to the epifluorescence ones.

\subsubsection{MOFs for biological fluorescence imaging}

Luminescent MOFs in the nano-regime can be an effective agent for biological imaging applications. Several advantages of nanoparticles over small-molecule imaging agents include their high payloads, tunable sizes, tailorable surface properties, and improved pharmacokinetics [182]. Recently, Kundu et al. reported a Gd ${ }^{\mathrm{III}}$-based MOF Gd-pDBI-2 (13) for the potential application as a bimodal imaging agent [140]. The motivation behind this work is to generate a MOF displaying fluorescence emitted by the organic ligand in combination with the magnetic resonance imaging (MRI) property of the $\mathrm{Gd}^{\mathrm{III}}$ ion. 13 was formed from the reaction between the fluorescent ligand 1,4-bis(5-carboxy-1Hbenzimidazole-2-yl)benzene (pDBI) and $\mathrm{Gd}(\mathrm{OAc})_{3}$ in an open conical flask at $120{ }^{\circ} \mathrm{C}$ for 3 hours. Due to the very small sizes of the crystals of $\mathbf{1 3}$, the single-crystal structure was not determined; however, the PXRD pattern of $\mathbf{1 3}$ was obtained to confirm the high crystallinity 
of the sample. The SEM and TEM images displayed spindle-like particles with dimensions of $\sim 1 \mu \mathrm{m}$ in length and $\sim 0.3 \mu \mathrm{m}$ in width. The MOF can be dispersed in water without affecting its stability, as verified by PXRD and FTIR studies. Luminescent studies showed that $\mathbf{1 3}$ exhibits a blue emission centered at $415 \mathrm{~nm}$ when excited under UV radiation; the maximum emission intensity was obtained with the $340 \mathrm{~nm}$ excitation light. Prior to bioimaging experiments, an MTT assay of $\mathbf{1 3}$ on MCF7 cell lines was performed giving the cellular viability of $88 \%$, which confirmed the low cytoxicity of the sample for imaging purposes. When MCF7 cells were incubated with $\mathbf{1 3}$ and observed under a confocal microscope, most of them exhibited bright blue-colored fluorescence at an excitation wavelength of $405 \mathrm{~nm}$ (Figure 9, c). The merged image (Figure 9, b) indicated the presence of $\mathbf{1 3}$ within the cytoplasm of the MCF7 cells. It is worth mentioning that although $\mathbf{1 3}$ also displays a modest longitudinal relaxivity $\mathrm{T}_{1}$ for the MRI application, the small-quantity leaking of Gd ions in an aqueous solution observed by the inductively coupled plasma optical emission spectrometry (ICP-OES) analysis showed that MOFs with higher stability are needed for practical applications.

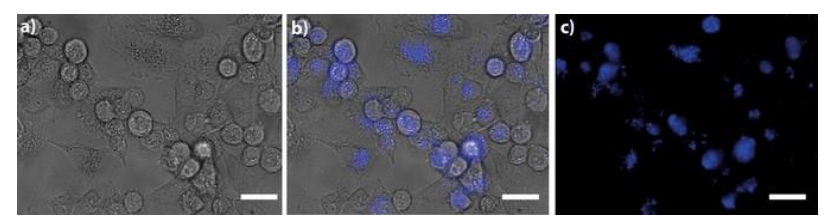

Figure 9. Confocal microscopic images of MCF7 cells incubated with $\mathbf{1 3}$ at an excitation wavelength of $405 \mathrm{~nm}$; bright-field image (a), merged image (b), fluorescence image (c). Reproduced with permission from reference [140].

Liu et al. reported a coordination polymer (14) constructed from the reaction between $\mathrm{ZrCl}_{4}$ and the phosphorescent ligand $\left[\mathrm{Ru}\left\{5,5^{\prime}-\left(\mathrm{CO}_{2}\right)_{2}-\mathrm{bpy}\right\}(\mathrm{bpy})_{2}\right]$ in a microwave-heating synthesis [141]. 14 is amorphous based on PXRD studies; SEM and TEM images showed 
that the spherical particles have an average diameter of $85 \mathrm{~nm}$, which agrees with the sizes obtained from dynamic light scattering (DLS) measurements. 14 exhibits a quantum yield of $0.8 \%$ and an average luminescence lifetime of $107 \mathrm{~ns}$. Due to the instability of $\mathbf{1 4}$ in phosphate-buffered saline solution at $370{ }^{\circ} \mathrm{C}$, the authors developed a method to coat the particles with a thin layer of silica to improve their biocompatibility. Silica coatings also provide surface silanol groups for further functionalizations; therefore 14@ $\mathrm{SiO}_{2}$ samples functionalized with polyethylene glycol (PEG) and anisamide-PEG (AA-PEG) were also prepared. Anisamide is a targeting ligand that has moderate affinity for sigma receptors; therefore, its attachment can improve the delivery of the particles to prostate and lung cancer cells [183]. Incubation of $\mathrm{H} 460$ cells with $14 @ \mathrm{PEG}^{-\mathrm{SiO}_{2}}$ and $14 @ \mathrm{AA}-\mathrm{PEG}-\mathrm{SiO}_{2}$ for 24 hours did not lead to appreciable cell death. Confocal fluorescence microscopy studies showed that a phosphorescent signal was observed in the image of $\mathrm{H} 460$ cells incubated with 14@AA-PEG-SiO $\mathrm{S}_{2}$ whilst the signal of $14 @ \mathrm{PEG}-\mathrm{SiO}_{2}$ was seen not only in the cells but also in the media as expected for the non-targeted 14@ PEG-SiO 2.

Foucault-Collet et al. designed a nanoscale Yb-based MOF (15) exhibiting NIR luminescence for imaging of living cells [142]. The nanoparticles were synthesized based on the reversemicroemulsion method using 4,4'-[(2,5-dimethoxy-1,4-phenylene)di-2,1-ethenediyl]bisbenzoate $\left(\mathrm{PVDC}^{2-}\right)$ as the ligand. SEM images showed that $\mathbf{1 5}$ appears as block microcrystals with dimensions of $0.5( \pm 0.3) \mu \mathrm{m}$ (length) by $316( \pm 156) \mathrm{nm}$ (width) by $176( \pm 52)$ nm (thickness). The bulk material was also obtained for single-crystal X-ray studies. The secondary building unit is an infinite chain of $\mathrm{Yb}^{\mathrm{III}}$ ions, which are octacoordinated with six carboxylates from three ligands and two oxygen atoms from two DMF molecules. Photophysical studies gave the quantum yield values for 15 of $(1.0 \pm 0.3) \times 10^{-4}$ in water and $(5.2 \pm 0.8) \times 10^{-5}$ in $0.1 \mathrm{M}$ Hepes buffer $(\mathrm{pH} 7.3)$ when the samples were excited at $450 \mathrm{~nm}$. 
These quantum yields are quite low, probably due to the exchange of DMF solvents by water molecules that quench the luminescence through multi-phonon processes. Nevertheless, photobleaching tests in combination with PXRD and SEM studies indicated that $\mathbf{1 5}$ is stable in these solutions and also in cellular media. In addition, cytotoxicity of $\mathbf{1 5}$ for HeLa and NIH $3 \mathrm{~T} 3$ cell lines was investigated and revealed that a concentration of $30 \mu \mathrm{g} / \mathrm{mL}$ of the nanoparticles give the cellular viability of $90 \%$, thus it can be used for bioimaging of these cells. Emission signals in the visible region originated from the chromophore PVDC ${ }^{2-}$ and in the NIR region stemmed from $\mathrm{Yb}^{\mathrm{III}}$ were clearly observed by epifluorescence microscopy (Figure 10), which supports the argument of the authors that using a MOF system to maximize the number of chromophores and lanthanide cations can reduce the negative effect of the low quantum yields.
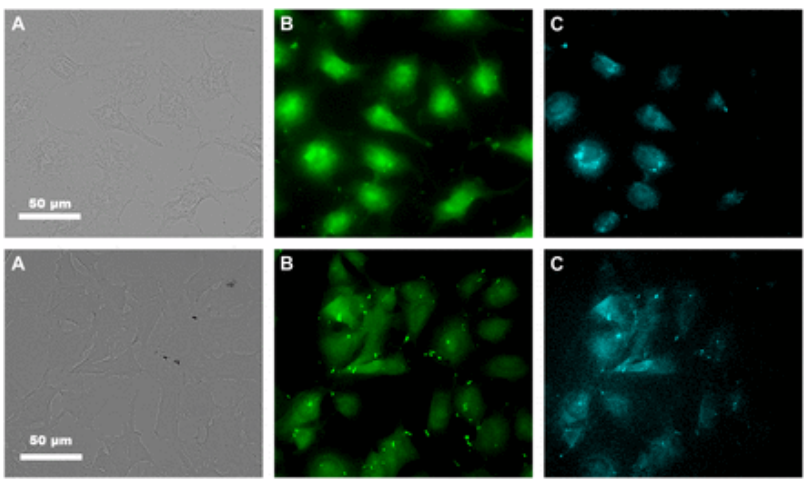

Figure 10. Visible and NIR microscopy images of $\mathbf{1 3}$ in HeLa cells (top) and NIH 3T3 cells (bottom) $\left(\lambda_{\mathrm{ex}}=340 \mathrm{~nm}\right)$. Bright-field (A), $\mathrm{PVDC}^{2-}$ emission $\left(\lambda_{\mathrm{ex}}=377 / 50 \mathrm{~nm}, \lambda_{\mathrm{em}}=445 / 50\right.$ $\mathrm{nm})(\mathrm{B})$, and $\mathrm{Yb}^{\mathrm{III}}$ emission $\left(\lambda_{\mathrm{ex}}=377 / 50 \mathrm{~nm}, \lambda_{\mathrm{em}}=\right.$ long pass $\left.770 \mathrm{~nm}\right)(\mathrm{C})$ images are shown. Reproduced with permission from reference [142].

Taylor-Pashow et al. reported a nanoscale Fe-based MOF (called MIL-101(Fe)) functionalized with amino groups to covalently graft the BODIPY dye through a postsynthetic modification [143]. The BODIPY-loaded particles (16) can have dye loading 
reaching $11.6 \mathrm{wt} \% .16$ is non-emissive due to luminescence quenching by the $\mathrm{d}$-d transitions of the paramagnetic $\mathrm{Fe}^{\mathrm{III}}$ centers. However, when these particles are put in a biological media, the BODIPY dye is released due to the degradation of the particles and the solution exhibits a fluorescence signal characteristic of the BDC-NH-BODIPY species. The rate of this release is quite fast, with a $\mathrm{t}_{1 / 2}$ of $\sim 2.5 \mathrm{~h}$ in $8 \mathrm{mM}$ PBS buffer at $37^{\circ} \mathrm{C}$. To reduce this rate, the author coated $\mathbf{1 6}$ with a layer of silica; the resulting 16@silica has the BODIPYrelease $\mathrm{t}_{1 / 2}$ of $\sim 16 \mathrm{~h}$ in PBS buffer at $37{ }^{\circ} \mathrm{C}$, which is long enough for biological imaging experiments. Confocal microscopy images of HT-29 human colon adenocarcinoma cells incubated with particles of $\mathbf{1 6} @$ silica showed fluorescence signals that are attributed to the BDC-NH-BODIPY dye slowly increasing its concentration in the cells. Interestingly, control studies with the BDC-NH-BODIPY dye itself without the MOF particles showed no fluorescence, presumably due to its inability to cross the cell membrane. Therefore, the nanosized particles of $\mathbf{1 6}$ are efficient carriers for delivering a fluorophore in vitro.

Encapsulation of phosphorescent metal complexes within MOFs can lead to luminescent composites for optical imaging. Chen et al. reported the preparation of UiO-67-type MOF nanoparticles (17) via a solvothermal reaction that can encapsulate $\left[\mathrm{Ru}(\mathrm{bpy})_{3}\right]^{2+}$ complexes up to $13.85 \%$ of their weight [144]. SEM and TEM images of the MOF itself showed spherical nanoparticles with an average diameter of $\sim 92 \mathrm{~nm}$. Incorporation of the $\mathrm{Ru}$ complex inside the pores of these nanoparticles does not significantly change their sizes. The composite 17 exhibits a broad luminescent emission centered at $621 \mathrm{~nm}$, which is blueshifted from that $(631 \mathrm{~nm})$ of the free $\mathrm{Ru}$ dye probably due to the interaction between the dye molecule and the MOF. The quantum yield of $\mathbf{1 7}$ is twice as high compared to value measured for $\left[\mathrm{Ru}(\mathrm{bpy})_{3}\right]^{2+}$, which was attributed to the large separation of the dyes within the MOF and the constraint of molecular motions that effectively decrease the quenching effects. 
Interestingly, 17 displays two-photon luminescence when is excited at $880 \mathrm{~nm}$. The MMT essay was performed to examine the cytotoxicity of $\mathbf{1 7}$ on A549 pulmonary cancer cells. The NPs show good biocompatibility at low concentrations; therefore, they were subjected to biological imaging experiments. Confocal laser scanning microscopy studies showed that the cells incubated with the NPs of $\mathbf{1 7}$ display clear red fluorescence when excited at $880 \mathrm{~nm}$, while those without the NPs only exhibit blue fluorescence. This result suggests that this composite can be used for two-photon luminescent imaging of biological objects.

\subsubsection{Luminescent Sensing}

\subsubsection{Fundamental principles}

Luminescent sensing is based on changes in photophysical properties of the sensor, which plays the role as a "signaling" unit that provides a signal after "binding" to the analyte of interest or after a change in a physical parameter such as temperature or pressure. The most commonly observed change is the luminescence intensity, although lifetime-based sensors have also been reported. In fact, the measurement of changes in luminescence lifetime is more robust since it does not depend on the concentration of the luminophore used in the sensor; however, the instrument needed for lifetime-based sensors is often more complicated.

Three important parameters of chemical sensors are the limit of detection, selectivity, and time of response. The limit of detection (LOD) is the lowest quantity of the analyte that can be distinguished from its absence, i.e. the blank value, with $99 \%$ confidence. It is the function of both signal strength (sensitivity) and signal stability, and is frequently used interchangeably with sensitivity. The LOD value can be determined from the equation: LOD $=3 \sigma / \mathrm{S}$, in which $\sigma$ is the standard deviation of the blank sample that is obtained by a large number of measurements of luminescence intensity in the absence of the analyte, and $\mathrm{S}$ is the 
slope of the calibration curve. The LOD/sensitivity of a sensor strongly depends on its affinity to the analyte. Selectivity is the ability of a sensor to measure a concentration of an analyte in the presence of other interfering substances. Time of response is the time required for a sensor output to change from its previous state to a final settled value within a tolerance band of the correct new value. A fast response time is required for a sensor to have a practical application.

Temperature luminescent sensors are often designed to have either a single or a double luminescent center. The former approach is based on the decrease of the luminescence intensities and lifetimes when the temperature is increased (or the increase of luminescence intensities and lifetimes upon cooling). This is due to the Boltzmann distribution, as at higher temperatures more vibration levels are available and hence more non-radiative decays can occur. The thermal quenching effect can be applied to almost all luminescent materials. However, the disadvantage of having a single luminescence center is that the dependence of luminescence intensity on the probe concentration, excitation, or detection efficiency and hence leading to poor accuracy. The latter is based on the principle of ratiometric luminescence that often involves an energy transfer between the two luminescent centers and has been demonstrated as the superior approach for luminescent thermometers. A good temperature luminescent sensor should have: i) high sensitivity, i.e. a large change of luminescence intensity or lifetime with temperature and ii) high thermal stability and photostability for long-term uses.

Luminescent sensors for analytes can be based on either quenching or enhancing of the luminescence intensity, leading to "turn-off" and "turn-on" sensors, respectively. In many cases, the quenching or enhancing effect can be attributed to the electronic nature of the 
analyte being detected. Paramagnetic metal cations such as $\mathrm{Cu}^{2+}, \mathrm{Fe}^{3+}$, or $\mathrm{Mn}^{2+}$ are wellknown quenchers for luminescence since they can induce LMCT or MLCT processes, which allow non-radiative relaxation of the excitation energy. Electron-poor organic molecules such as nitro explosives are also strong luminescence quenchers since their low energy unoccupied $\pi^{*}$ orbitals have high electron affinity leading to electron transfers of the excited electrons and hence decreasing the density of excited electrons without luminescent emission. On the contrary, electron-rich molecules can sometimes enhance luminescence due to their ability to donate electrons to the excited states of the luminescent sensor material. Several "turn-on" sensors can be designed based on the photo-induced electron transfer (PeT) effect. These sensors are also termed "reactive probes" since they operate based on irreversible chemical reactions between the sensor and the targeting analyte. These reactive probes often exhibit higher sensitivity and selectivity compared to sensors based on non-covalent interactions owing to the structural changes from the chemical conversion.

\subsubsection{MOFs for luminescent sensing}

\subsection{MOFs as optical thermometers}

Temperature sensors are among the most common sensors used in both scientific and industrial fields. MOF thermometers have been fabricated based on the principle of ratiometric luminescence. These MOFs exhibit dual emission from the combination of two organic luminophores or two lanthanide ions or by encapsulating organic dye molecules within lanthanide frameworks. Recently, Zhang et al. reported a Zn-based MOF (18) constructed from the mixture of two ligands, $\mathrm{BTB}^{3-}$ and ATZ (5-amino-1-H-tetrazolate) [145]. 18 displays temperature-dependent emission behavior under near-UV excitation, with LLCT emission bands of the ATZ ligands at 477, 510, and $544 \mathrm{~nm}$ appearing while the ILCT emission band of the $\mathrm{BTB}^{3-}$ ligand at $377 \mathrm{~nm}$ maintains its intensity when lowering the 
temperature to $10 \mathrm{~K}$ (Figure 11, top). A linear relationship between the intensity ratio and temperature from $30 \mathrm{~K}$ to $130 \mathrm{~K}$ was established: $\mathrm{T}=10.80\left(\mathrm{I}_{\mathrm{HE}} / \mathrm{I}_{\mathrm{LE}}\right)+11.52$ (Figure 11 , bottom). In addition, the absolute and relative sensitivities, defined as $S_{a b}=\frac{\partial\left(\frac{I_{H E}}{I_{L E}}\right)}{\partial T}$ and $S_{r e}=$ $\frac{\partial\left(\frac{I_{H E}}{I_{L E}}\right) / \partial T}{I_{H E} / I_{L E}}$, are $0.0926 \mathrm{~K}^{-1}$ and $5.29 \% \mathrm{~K}^{-1}$ at $30 \mathrm{~K}$, which are quite high, indicating that $\mathbf{1 8}$ can be used as a luminescent thermometer for the cryogenic temperature range (30-130 K).
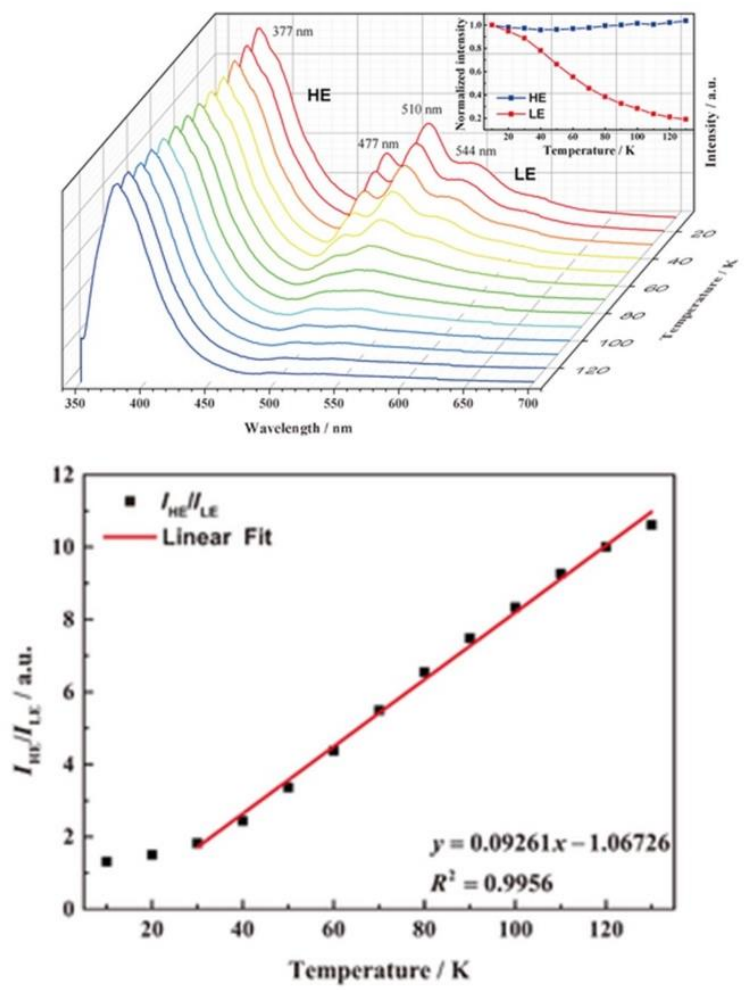

Figure 11. (Top) Emission spectra of $\mathbf{1 8}$ recorded between 10 and $130 \mathrm{~K}$ under excitation at $330 \mathrm{~nm}$. Inset: the temperature-dependent integrated intensity of the HE and LE emissions. (Bottom) The linear-fitted line for the temperature-dependent intensity ratio of the HE and LE transitions. Reproduced with permission from reference [145].

Yang et al. designed a doped $\mathrm{Eu}_{\mathrm{x}} \mathrm{Tb}_{1-\mathrm{x}} \mathrm{MOF}(19)$ that was formed from the use of 2,6$\operatorname{di}\left(2^{\prime}, 4^{\prime}\right.$-dicarboxylphenyl)pyridine for temperature sensing [146]. With low concentration of 
Eu ${ }^{\mathrm{III}},(\mathrm{x}=0.0066$ and 0.0089$)$, the emission intensity of $\mathrm{Tb}^{\mathrm{III}}$ at $542 \mathrm{~nm}$ decreases dramatically while that of $\mathrm{Eu}^{\mathrm{III}}$ at $612 \mathrm{~nm}$ increases significantly at the same time over the temperature range of $77-450 \mathrm{~K}$. A linear relationship between the temperature and the emission intensity ratios was established, $\mathrm{I}_{\mathrm{Tb}} / \mathrm{I}_{\mathrm{Eu}}=13.516-0.0319 \mathrm{~T}$ and $\mathrm{I}_{\mathrm{Tb}} / \mathrm{I}_{\mathrm{Eu}}=9.107-$ $0.0207 \mathrm{~T}$ for $\mathrm{x}=0.0066$ and 0.0089 , with the corresponding relative sensitivities at $450 \mathrm{~K}$ of $3.76 \% \mathrm{~K}^{-1}$ and $2.71 \% \mathrm{~K}^{-1}$, respectively. Although these values are not excellent, this work illustrated the potential of $\mathbf{1 9}$ as a luminescent thermometer for a pretty wide range of temperature, including the cryogenic region (100-298 K), the physiological temperature (298-320 K), and the high temperature range (320-450 K). Interestingly, the authors also investigated the ternary mixed lanthanide MOF by doping with $\mathrm{Gd}^{\mathrm{III}}$. The doped MOF with the ratio of $\mathrm{Eu}: \mathrm{Tb}: \mathrm{Gd}$ of $0.013: 0.060: 0.927$ display the maximum relative sensitivity of $6.11 \% \mathrm{~K}^{-1}$ at $430 \mathrm{~K}$. This relatively high sensitivity suggests that tertiary lanthanide MOFs might be the future targets for ratiometric luminescent thermometers with high performance.

Lian et al. adapted the concept of using mixed lanthanide MOFs as luminescent thermometers but employed NIR lanthanide ions in place of the common visible luminescent $\mathrm{Eu}^{\mathrm{III}} / \mathrm{Tb}^{\mathrm{III}}$ ions. Optical thermometers based on NIR luminescence will be practically very useful because NIR emission will have high permeability through biotissues and no competing fluorescence from the background [147]. The MOF (20) was prepared by reacting $\mathrm{Nd}\left(\mathrm{NO}_{3}\right)_{3} \cdot 6 \mathrm{H}_{2} \mathrm{O}$ and $\mathrm{Yb}\left(\mathrm{NO}_{3}\right)_{3} \cdot 5 \mathrm{H}_{2} \mathrm{O}$ with tetrafluoroterephthalic acid $\left(\mathrm{H}_{2} \mathrm{BDC}-\mathrm{F}_{4}\right)$ to obtain the product with the formula of $\left[\left(\mathrm{Nd}_{0.577} \mathrm{Yb}_{0.423}\right)_{2}\left(\mathrm{BDC}-\mathrm{F}_{4}\right)_{3}(\mathrm{DMF})\left(\mathrm{H}_{2} \mathrm{O}\right)\right] \cdot \mathrm{DMF}$. The mono lanthanide MOFs, $\left[\mathrm{Nd}_{2}\left(\mathrm{BDC}-\mathrm{F}_{4}\right)_{3}(\mathrm{DMF})\left(\mathrm{H}_{2} \mathrm{O}\right)\right] \cdot \mathrm{DMF} \quad(\mathbf{2 0 - N d})$ and $\quad\left[\mathrm{Yb}_{2}(\mathrm{BDC}-\right.$ $\left.\left.\mathrm{F}_{4}\right)_{3}(\mathrm{DMF})\left(\mathrm{H}_{2} \mathrm{O}\right)\right] \cdot \mathrm{DMF}(\mathbf{2 0}-\mathbf{Y b})$ were also obtained. Upon laser pumping at $808 \mathrm{~nm}, \mathbf{2 0}-\mathbf{N d}$ exhibits emissions at around $890 \mathrm{~nm}, 1060 \mathrm{~nm}$ and $1340 \mathrm{~nm}$, which are the characteristic f-f transitions of $\mathrm{Nd}^{\mathrm{III}}$. 20-Yb does not absorb light at $808 \mathrm{~nm}$ and therefore no emission was 
observed. The mixed lanthanide MOF (20), however, displays emissions of both $\mathrm{Nd}^{\mathrm{III}}$ and $\mathrm{Yb}^{\mathrm{III}}$, implying that energy transfer from $\mathrm{Nd}^{\mathrm{III}}$ to $\mathrm{Yb}^{\mathrm{III}}$ occurred (Figure 12). When temperature is increased from 293 to $313 \mathrm{~K}$, the intensity of the $980 \mathrm{~nm}$ emission of $\mathrm{Yb}^{\mathrm{III}}$ $\left({ }^{2} \mathrm{~F}_{5 / 2}-{ }^{2} \mathrm{~F}_{7 / 2}\right)$ also increases rapidly while the intensity of the $1060 \mathrm{~nm}$ emission of $\mathrm{Nd}^{\mathrm{III}}\left({ }^{4} \mathrm{~F}_{3 / 2}\right.$ - ${ }^{4} \mathrm{I}_{11 / 2}$ ) remains the same until $308 \mathrm{~K}$ and then slightly increases afterwards. A linear relationship between the $\mathrm{I}_{\mathrm{Nd}} / \mathrm{I}_{\mathrm{Yb}}$ ratio and the temperature was found, $\mathrm{T}=434.67$ $143.27 \mathrm{I}_{\mathrm{Nd}} / \mathrm{I} \mathrm{Yb}$, and the maximum relative sensitivity is of $0.816 \% \mathrm{~K}^{-1}$ at $313 \mathrm{~K}$. The

calculated temperature resolution is $0.029 \mathrm{~K}$, suggesting the potential use of $\mathbf{2 0}$ for biomedical diagnosis since the temperature difference between pathological cells and normal surrounding cells is around 0.5-3.3 K [147].

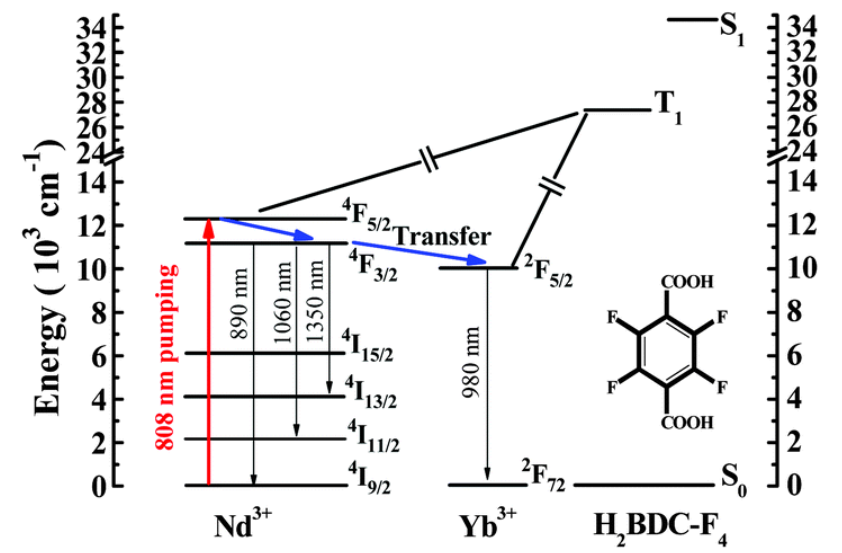

Figure 12. Energy transfer from $\mathrm{Nd}^{\mathrm{III}}$ to $\mathrm{Yb}^{\mathrm{III}}$ upon laser pumping at $808 \mathrm{~nm}$. Reproduced with permission from reference [147].

Although mixed lanthanide MOFs such as $\mathbf{1 9}$ and $\mathbf{2 0}$ are good candidates for luminescent thermometry, Xia et al. argued that these MOFs will lose the sensing ability at elevated temperatures due to the saturation of the energy transfer between the lanthanide ions [148]. They proposed that dual emissions can be generated from the two thermally coupled energy levels (TCELs) of a single lanthanide ion. A Dy-based MOF (21) was synthesized from the 
reaction between 5-(4-carboxyphenoxy)isophthalic acid $\left(\mathrm{H}_{3}\right.$ cpia) and $\mathrm{Dy}\left(\mathrm{NO}_{3}\right)_{3} \cdot 6 \mathrm{H}_{2} \mathrm{O}$ via a solvothermal method. 21 exhibits main emissions at 487, 574, and $661 \mathrm{~nm}$ which are characteristic f-f transitions of Dy ${ }^{\mathrm{III}}$. Upon increasing the temperature, the intensity of the transition at $455 \mathrm{~nm}\left({ }^{4} \mathrm{I}_{15 / 2}-{ }^{6} \mathrm{H}_{15 / 2}\right)$ increased while the intensity of the emission at $485 \mathrm{~nm}$ $\left({ }^{4} \mathrm{~F}_{9 / 2}{ }^{6} \mathrm{H}_{15 / 2}\right)$ slightly decreases. Based on the TCEL theory, in which the population of the two TCELs is governed by the Boltzmann distribution law, the relationship between the intensity ratio of the luminescence originating from these two TCELs and temperature is given by the equation: $\mathrm{I}_{1} / \mathrm{I}_{2}=4.65 \exp (-1837.1 / \mathrm{T})+0.091$. The absolute sensitivities are found to be $2.02 \times 10^{-4} \mathrm{~K}^{-1}$ at $298 \mathrm{~K}$ and $7.85 \times 10^{-4} \mathrm{~K}^{-1}$ at $473 \mathrm{~K}$ whilst the maximum relative sensitivity is $0.42 \% \mathrm{~K}^{-1}$ at $473 \mathrm{~K}$. Although these sensitivities are pretty low, the author demonstrated the repeatability of $\mathbf{2 1}$ as a luminescent thermometer for at least 12 heatingcooling cycles (Figure 13), which is not often seen in other reports [148].

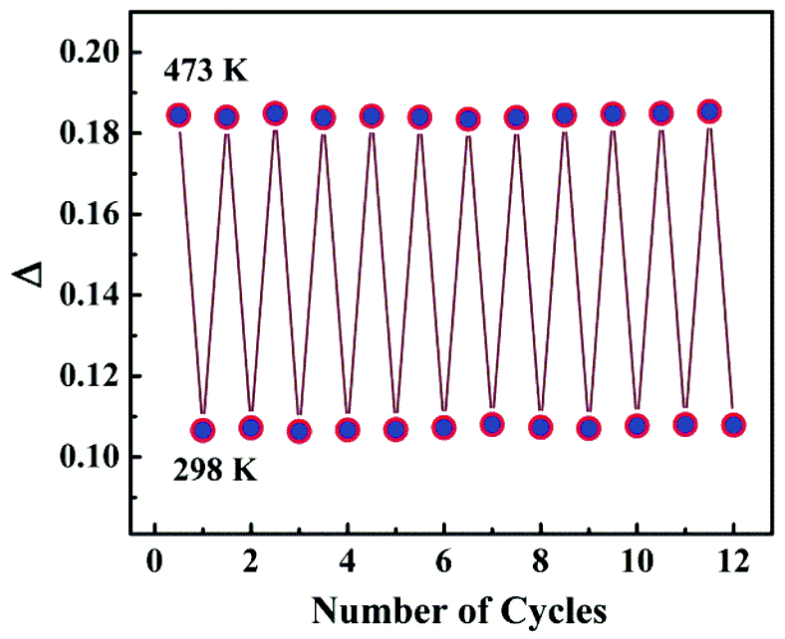

Figure 13. The reversible emission intensity ratio of the ${ }^{4} \mathrm{I}_{15 / 2}-{ }^{6} \mathrm{H}_{15 / 2}$ transition to the ${ }^{4} \mathrm{~F}_{9 / 2}$ ${ }^{6} \mathrm{H}_{15 / 2}$ transition between $298 \mathrm{~K}$ and $473 \mathrm{~K}$. Reproduced with permission from reference [148]. 
Departing from the use of mixed lanthanide MOFs, Cui et al. employed the encapsulation of the organic dye perylene within a Eu-based MOF to obtain a dual-emitting composite (22) for temperature sensing [149]. The MOF (also called ZJU-88) was formed from the use of $1,1^{\prime}: 4^{\prime}, 1^{\prime \prime}: 4^{\prime \prime}, 1^{\prime \prime \prime}$-quaterphenyl-3,3'",5,5'"-tetracarboxylate as the ligand. The composite 22 features a red emission of $\mathrm{Eu}^{\mathrm{III}}$ at $615 \mathrm{~nm}$ and a blue emission around $473 \mathrm{~nm}$ of perylene dyes when excited at $388 \mathrm{~nm}$. The emission peak profile of perylene dye in $\mathbf{2 2}$ is similar to that of the perylene solution but is significantly different from the one in the solid state, suggesting that the dye is distributed in the void channels of the MOF as free isolated molecules. When $\mathbf{2 2}$ is subjected to the variable temperature luminescence measurement in the range of $20-80{ }^{\circ} \mathrm{C}$, the luminescence intensity at $473 \mathrm{~nm}$ of the dye substantially decreases while the intensity of the $615 \mathrm{~nm}$ emission of $\mathrm{Eu}^{\mathrm{III}}$ increases. This can be attributed to the energy transfer from the dye molecules to the $\mathrm{Eu}^{\mathrm{III}}$ ions, as evidenced from the concomitant increase of the luminescence lifetime of the $\mathrm{Eu}^{\mathrm{III}}$ ions and the decrease of the luminescence lifetime of the perylene dye within 22. A linear relationship between the temperature and the emission intensity ratios at $615 \mathrm{~nm}\left(\mathrm{Eu}^{\mathrm{III}}\right)$ and $473 \mathrm{~nm}$ (perylene) was established, $T=57.53$ $-78.01 I_{615} / I_{473}$, with the maximum relative sensitivity of $1.28 \% \mathrm{C}^{-1}$ at $20{ }^{\circ} \mathrm{C}$. Although the working temperature range is rather narrow, $20-80{ }^{\circ} \mathrm{C}$, the authors suggested that 22 might still be useful in the biomedical diagnosis due to its low toxicity and good biocompatibility.

\subsection{MOFs as $\mathrm{pH}$ meters}

The measurement of $\mathrm{pH}$ value is crucial in a wide range of applications from environmental, industrial to biomedical fields, especially for monitoring $\mathrm{pH}$ changes in biological environments. Different from electrochemical sensors, luminescent $\mathrm{pH}$ sensors are nondestructive to cells. They often contain a weak acidic or basic group that undergoes reversible changes in the optical properties upon protonation and deprotonation. For example, Aguilera- 
Sigalat et al. realized that the well-known MOF UiO-66- $\mathrm{NH}_{2}$, which is formed by linking $\mathrm{Zr}_{6}$ clusters with the ligand 2-aminoterephthalate $\left(\mathrm{NH}_{2}-\mathrm{BDC}\right)$, displays the $\mathrm{pH}$-dependent fluorescence up to $\mathrm{pH}=9$. This $\mathrm{pH}$-dependent fluorescence is due to the easy pronation of the group $-\mathrm{NH}_{2}$ to $-\mathrm{NH}_{3}{ }^{+}$. To increase the MOF stability in the basic media, the authors partially incorporated indole moieties $(\sim 70 \%)$ into the framework by post synthetic modification via diazotization of the amino groups (23) [150]. 23 exhibits high stability and maintains its crystallinity and porosity up to $\mathrm{pH}=12$, which were attributed to the extended delocalization of the arylazo group. Fluorescence measurements revealed that the intensity increases with increasing $\mathrm{pH}$ (Figure 14) allowing $\mathbf{2 3}$ to be potentially used as a $\mathrm{pH}$ sensor.

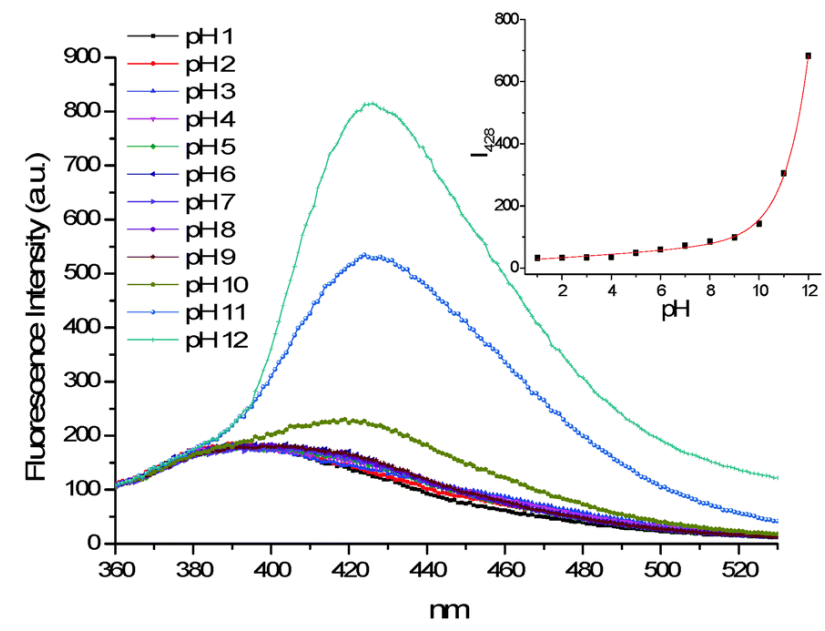

Figure 14. Normalized fluorescence emission of $\mathbf{2 3}$ vs. pH. Inset: exponential correlation between the fluorescence response of 23 and $\mathrm{pH}\left(\lambda_{\mathrm{ex}}=350 \mathrm{~nm}, \lambda_{\mathrm{em}}=428 \mathrm{~nm}\right)$. Reproduced with permission from reference [150].

$\mathrm{Xu}$ et al. employed a similar strategy as above by using Al-MIL-101- $\mathrm{NH}_{2}$ (24) for sensing $\mathrm{pH}$ in aqueous environments [151]. 24 exhibits good stability in water and remains intact in both basic and acidic aqueous solutions. When excited at $396 \mathrm{~nm}$, the crystalline powder of 24 displays a bright blue luminescence $\left(\lambda_{\mathrm{em}}=451 \mathrm{~nm}\right)$, which can also be observed when these 
powder samples are dispersed in $\mathrm{NaOH}$ or $\mathrm{HCl}$ aqueous solutions with $\mathrm{pH}$ ranging from 7.7 to 4.0. The fluorescence intensity rises along with $\mathrm{pH}$ increase and a linear relationship between them were established: $\mathrm{I}=2.33 \mathrm{pH}+26.04$. The author also investigated the possibility of using 24 to measure the $\mathrm{pH}$ of phosphate buffered saline solutions and of a water sample obtained from a nearby lake. A good $\mathrm{pH}$-fluorescence intensity linear relationship was observed for the former case suggesting that $\mathbf{2 4}$ is promising for uses in biological samples. However, in the latter case, no good relationship was obtained, which was attributed to the presence of metal ions such as $\mathrm{Fe}^{\mathrm{III}}, \mathrm{Mn}^{\mathrm{II}}, \mathrm{Cr}^{\mathrm{II}}$ in the lake water sample that can bind to the $-\mathrm{NH}_{2}$ groups of the $\mathrm{MOF}$ and alter its fluorescence property.

Lanthanide-based MOFs are often quite stable in aqueous solutions; therefore, they are good candidates as $\mathrm{pH}$ sensors. Li et al. utilized the organic ligand derived from 1-(3,5dicarboxybenzyl)-4,4'-bipyridinium chloride $\left(\mathrm{H}_{2} \mathrm{~L}^{+} \mathrm{Cl}^{-}\right)$to synthesize a luminescent $\mathrm{Tb}$-based MOF (25) in which the pyridyl nitrogen atoms can undergo protonation under acidic conditions [152]. 25 remains intact in aqueous solutions with $\mathrm{pH}$ ranging from 2 to 7; therefore, the powdered samples of $\mathbf{2 5}$ were subjected to the examination of $\mathrm{pH}$ influences on the luminescence intensity. The solid-state emission of the acid-treated samples gradually decreased in intensity when lowering the $\mathrm{pH}$, with a linear response $\mathrm{I}=0.0596 \times \mathrm{pH}+0.5871$ being observed within the range of $\mathrm{pH}=2-7$. The luminescent sensitivity to the $\mathrm{pH}$ was attributed to the change in the electron withdrawing ability of the pendant viologen group, which may be responsible for the decrease of intensity of luminescence at low $\mathrm{pH}$.

Incorporating a lanthanide ion into a water-stable MOF is another strategy to construct $\mathrm{pH}$ sensors. Zhang et al. reported the formation of a Eu@UiO-67-bpydc MOF (26) by simply heating the mixture of $\mathrm{UiO}-67-b p y d c$ with $\mathrm{Eu}\left(\mathrm{NO}_{3}\right)_{3} \cdot 6 \mathrm{H}_{2} \mathrm{O}$ in $\mathrm{MeOH}$ at $60{ }^{\circ} \mathrm{C}$ [153]. A small 
fraction of the bipyridine groups coordinates to $\mathrm{Eu}^{\mathrm{III}}$ ions; the remaining bipyridines are free and can be protonated in acidic solutions (Figure 15). The crystalline powder of $\mathbf{2 6}$ exhibits luminescent lines characteristic of the $\mathrm{Eu}^{\mathrm{III}}$ ion, and the luminescence intensity is strongly correlated with the $\mathrm{pH}$ value of the solution that it is dispersed in. Similar to the above examples, the most acidic solution $(\mathrm{pH}=1.06)$ gave the weakest luminescence while the highest intensity is obtained with $\mathrm{pH}=7.6$ solution. The author also performed the MTT assay by incubating pheochromocytoma (PC12) cells with 26 at doses ranging from 5 to 100 $\mu \mathrm{g} / \mathrm{mL}$ for $24 \mathrm{~h}$. In all cases, the cell viability is still above $80 \%$, indicating the potential of 26 for $\mathrm{pH}$ sensing in biological samples.
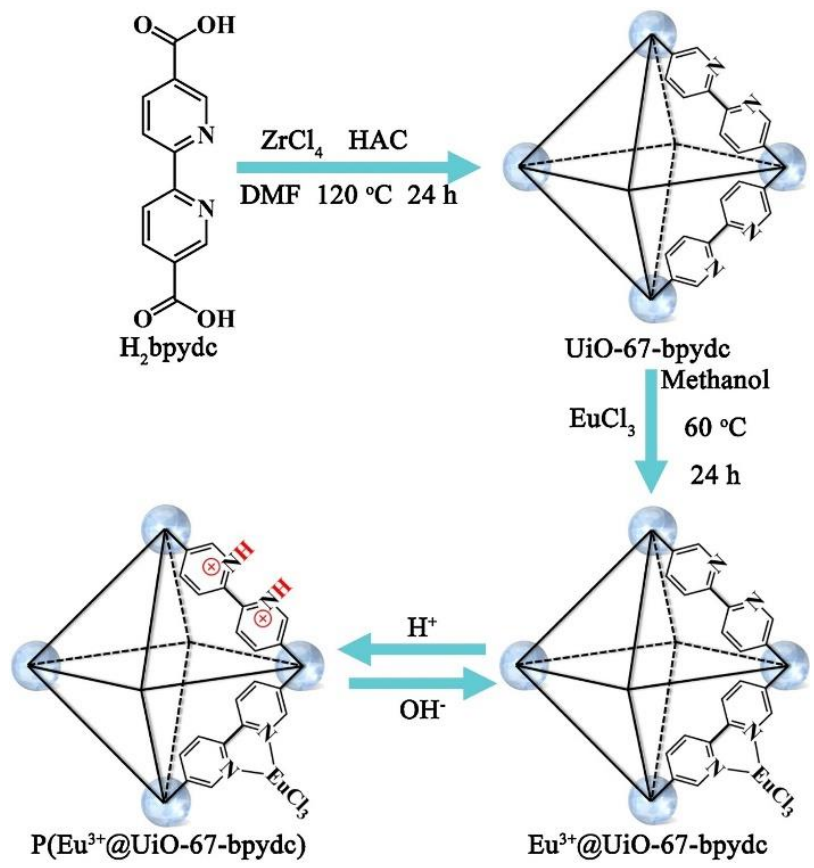

Figure 15. The post-synthetic $\mathrm{Eu}^{\mathrm{III}}$ functionalization of UiO-67-bpydc, and protonation and deprotonation processes of nitrogen involved in UiO-67-bpydc framework in experimental acidic and basic media. Reproduced with permission from reference [153].

Wang et al. suggested that $\mathrm{pH}$ probes utilizing the changes of the intensity of a single emission have low accuracy due to the environmental interferences such as fluctuations in the 
source intensity or photobleaching of the sensors [154]. Ratiometric sensing, which is based on recording the ratio of two emissions at different wavelengths as the detecting signal, would be a superior approach. The authors utilized the organic ligand 5,5'-(ethane-1,2diylbis(oxy))diisophthalate $\left(\mathrm{EDDA}^{4-}\right)$ containing a diether group to construct a luminescent Cd-based 3-dimensional MOF (27). The ether bond moiety of EDDA ${ }^{4-}$ is used as a binding site for binding $\mathrm{H}^{+}$or $\mathrm{OH}^{-}$via hydrogen-bonding interactions. 27 exhibits a dual emission at 350 and $410 \mathrm{~nm}$ that can be assigned to the charge transfer between the ligand and the metal ions and the ligand-based emission, respectively. When 27 is excited at $310 \mathrm{~nm}$ and in an acidic environment, the intensity of the emission at $350 \mathrm{~nm}$ gradually increased on decreasing the $\mathrm{pH}$ from 6.5 to 2.0 , while the emission at $410 \mathrm{~nm}$ almost disappeared. From the calibration curve of the emission ratio $\mathrm{I}_{350} / \mathrm{I}_{410} \mathrm{vs}$. $\mathrm{pH}$, an apparent $\mathrm{pKa}$ of 4.34 can be assigned for $\mathbf{2 7}$ for this $\mathrm{pH}$ range. In the basic environment, when the $\mathrm{pH}$ is increased from 9.5 to 11.5 , the intensity of the emission at $350 \mathrm{~nm}$ increased concomitantly with the decrease of the emission intensity at $410 \mathrm{~nm}$. Similarly, the analysis of relative ratiometric intensities as a function of $\mathrm{pH}$ yielded a $\mathrm{pKa}$ value of 10.59 for this $\mathrm{pH}$ range. In addition, 27 can be regenerated by immersing it in a $\mathrm{NaCl}$ buffer solution, suggesting that $\mathbf{2 7}$ is a promising recyclable $\mathrm{pH}$ probe in aqueous solution.

Another recent attempt of designing luminescent ratiometric $\mathrm{pH}$ sensors is from Xia et al., who synthesized a nanoscale $\mathrm{Eu} / \mathrm{Tb}$-mix $\mathrm{MOF}(\mathbf{2 8})$ from the use of fumarate and oxalate as the ligands [155]. The framework 28, with the $\mathrm{Eu}^{\mathrm{III}}: \mathrm{Tb}^{\mathrm{III}}$ ratio of 0.034: 0.966, exhibits emission peaks characterized for $\mathrm{Eu}$ and $\mathrm{Tb}$ upon excitation at $340 \mathrm{~nm} . \mathbf{2 8}$ displays high stability and $\mathrm{pH}$-dependence luminescence in the $\mathrm{pH}$ range 3-7 as shown in Figure 16. The ratiometric parameter $\Delta\left(\Delta=I_{\mathrm{Eu}} / I_{\mathrm{Tb}} ; I_{\mathrm{Tb}}\right.$ and $I_{\mathrm{Eu}}$ are the emission intensities of Eu ${ }^{\mathrm{III}}$ at $618 \mathrm{~nm}$ and $\mathrm{Tb}^{\mathrm{III}}$ at $545 \mathrm{~nm}$ ) increases together with the increase of $\mathrm{pH}$ and is fit for the equation $\Delta=$ 
$1.207-48.13 e^{(-1.500 \times \mathrm{pH})}$. The $\mathrm{pH}-\mathrm{dependent} \mathrm{luminescence} \mathrm{emissions} \mathrm{in} \mathbf{2 8}$ are attributed to the hindrance of the energy transfer process by the hydronium ions, leading to the quenching of emissions at low $\mathrm{pH}$. The ${ }^{5} \mathrm{D}_{4} \rightarrow{ }^{7} \mathrm{~F}_{5}$ transition of $\mathrm{Tb}^{\mathrm{III}}$ is almost insensitive to the local environment while the change of the hypersensitive ${ }^{5} \mathrm{D}_{0} \rightarrow{ }^{7} \mathrm{~F}_{2}$ transition of Eu ${ }^{\mathrm{III}}$ is more significant leading to the dependence of $\Delta$ on $\mathrm{pH}$. The authors also demonstrated the low cytotoxicity and good biocompatibility of $\mathbf{2 8}$ with PC12 cells suggesting that $\mathbf{2 8}$ can be a potential $\mathrm{pH}$ sensor in physiological environments.

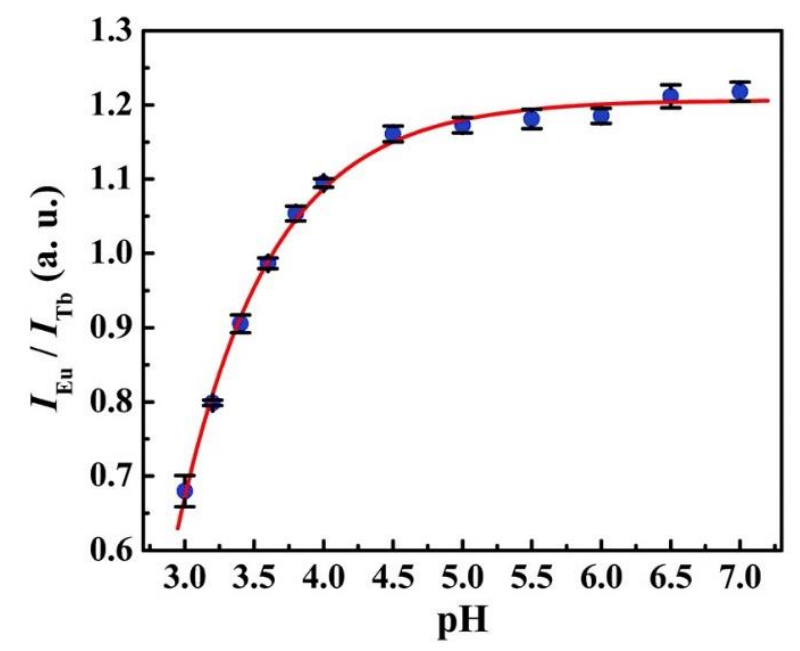

Figure 16. The fitted curve of $I_{\mathrm{Eu}} / I_{\mathrm{Tb}} \mathrm{vs} \mathrm{pH}$. Reproduced with permission from reference [155].

In summary, luminescent MOFs can be used for measuring $\mathrm{pH}$ of common aqueous solutions and biological media. However, as shown in all aforementioned MOF examples, these pH sensors can only perform in a rather small range of $\mathrm{pH}$, which might still be useful under certain circumstances, but in general they are not ideal for practical applications. The challenges in designing MOF-based $\mathrm{pH}$ sensors remain, including: i) the MOF should be stable in a wide range of $\mathrm{pH}$, ii) the MOF should be sensitive to $\mathrm{pH}$ changes but at the same time not affected by the presence of interferences such as metal ions, anions or biological 
compounds, and iii) it is preferable to have ratiometric sensing that allows for more accurate $\mathrm{pH}$ measurements.

\subsection{Sensing of metal ions}

Metal ions play critical roles in biology, with some of them being essential for fundamental processes such as metabolism, biomineralization, or catalysis [184]. Iron, zinc, copper, and other transition metals are all found in living biological systems as trace elements. Luminescent sensors for metal ions often contain a chelating group (not always) and a luminescent moiety that exhibits a change in its luminescence intensity or lifetime upon binding to a specific metal of interest. In the following sections, we separate metal ions into

two groups based on their magnetic property: paramagnetic ions, e.g. $\mathrm{Cu}^{\mathrm{II}}, \mathrm{Fe}^{\mathrm{III}}, \mathrm{Mn}^{\mathrm{II}}$ and diamagnetic ions, e.g. $\mathrm{Hg}^{\mathrm{II}}, \mathrm{Pb}^{\mathrm{II}}, \mathrm{Ag}^{\mathrm{I}}, \mathrm{Cd}^{\mathrm{II}}$.

\subsection{Paramagnetic ions}

Paramagnetic metal cations are generally luminescent quenchers; the mechanism of quenching is via either an energy- or a charge-transfer process based on $\mathrm{d}-\mathrm{d}$ transitions [35]. Therefore, most MOFs reported for sensing of these ions are "turn-off" sensors. For example, Bhattacharyya et al. reported the synthesis of a 3D anionic MOF (29) based on napthalenedicaboxylate $\left(\mathrm{ndc}^{2-}\right)$ ligand as a "turn-off" sensor for $\mathrm{Cu}^{\mathrm{II}}[156]$. Upon excitation at $317 \mathrm{~nm}, 29$ exhibits ndc-based emission at $\sim 410 \mathrm{~nm}$ in the solid state. 29 contains 1D channels occupied by the cationic guest $\mathrm{Me}_{2} \mathrm{NH}_{2}{ }^{+}$(DMA) that can be replaced by $\mathrm{Cu}^{\mathrm{II}}, \mathrm{Co}^{\mathrm{II}}$, and $\mathrm{Ni}^{\mathrm{II}}$ ions with the extent of 88,4 , and $12 \%$, respectively. Interestingly, no exchange was found for $\mathrm{Mn}^{\mathrm{II}}$ and $\mathrm{Zn}^{\mathrm{II}}$ ions. UV-Vis studies suggest the coordination of $\mathrm{Cu}^{\mathrm{II}}$ with the carboxylate oxygen atoms of the ligands, which leads to structural reorganization of the MOF and as a consequence, the PXRD pattern of the product after encapsulation of $\mathrm{Cu}^{\mathrm{II}}$ displays few new peaks. Although the mechanism of the selective capture of $\mathrm{Cu}^{\mathrm{II}}$ in $\mathbf{2 9}$ was unclear, 
the authors explained this phenomenon by the flexible geometry of $\mathrm{Cu}^{\mathrm{II}}$, which is distinct from other transition metals. The encapsulation of $\mathrm{Cu}^{\mathrm{II}}$ results in quenching of the fluorescent emission, with the fluorescence intensity decreasing when the concentration of $\mathrm{Cu}^{\mathrm{II}}$ in solution is increased (Figure 17). From the Stern-Völmer plot, which is based on the equation $I_{\mathrm{o}} / I=1+K_{\mathrm{SV}}[\mathrm{Q}]$ where $I_{\mathrm{o}}$ and $I$ are the initial and the quenched fluorescence intensities, $K_{\mathrm{SV}}$ is the Stern-Völmer constant (the higher the $K_{\mathrm{SV}}$, the more sensitive the sensor), and [Q] is the concentration of the quencher, the $K_{\mathrm{SV}}$ constants at 30 and $80{ }^{\circ} \mathrm{C}$ were found to be $1.986 \times$ $10^{3} \mathrm{M}^{-1}$ and $3.621 \times 10^{2} \mathrm{M}^{-1}$, respectively. This suggests that the quenching follows a static mechanism that is in agreement with the complexation of $\mathrm{Cu}^{\mathrm{II}}$ ions with the ligands of the MOF.

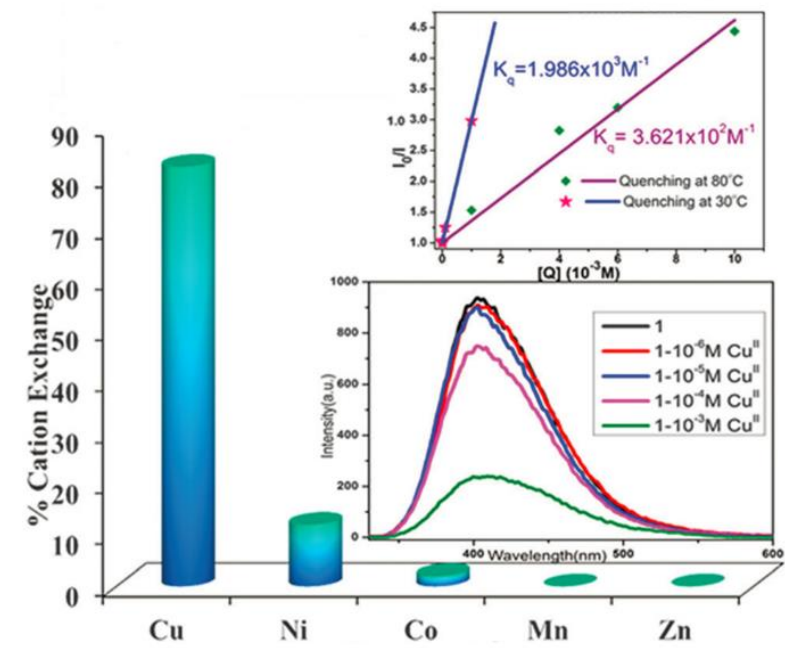

Figure 17. Uptake of different metal cations by $\mathbf{2 9}$ from an equimolar mixture of all the cations. Insets: (top) Stern- Völmer plot for quenching by $\mathrm{Cu}^{\mathrm{II}}$ at 30 and $80{ }^{\circ} \mathrm{C}$, (bottom) emission of 29 quenched by different concentrations of $\mathrm{Cu}^{\mathrm{II}}$ solutions. Reproduced with permission from reference [156].

Detection of paramagnetic cations with high selectivity is challenging since most of them quench luminescence in certain degrees. Another example of a "turn-off" sensor for metal 
ions was reported by Carboni et al. [157]. The Zr-based MOF (30) was synthesized from the use of succinamide-p,p'-terphenyldicarboxylic acid $\left(\mathrm{H}_{2} \mathrm{~L}\right)$, with the free carboxylic groups of the succinamide moiety present within the MOF channel expected to have high affinity towards transition metal cations. 30 displays a ligand-based broad emission band at $390 \mathrm{~nm}$ when excited at $330 \mathrm{~nm}$. When suspensions of $\mathbf{3 0} \mathrm{in} \mathrm{MeOH}$ were treated with different metal ions $\left(\mathrm{M}^{\mathrm{II}}=\mathrm{Fe}^{\mathrm{II}}, \mathrm{Mn}^{\mathrm{II}}, \mathrm{Co}^{\mathrm{II}}, \mathrm{Cd}^{\mathrm{II}}, \mathrm{Cu}^{\mathrm{II}}, \mathrm{Ni}^{\mathrm{II}}, \mathrm{Zn}^{\mathrm{II}}\right.$, and $\left.\mathrm{Mg}^{\mathrm{II}}\right)$, strong fluorescence quenching was observed for $\mathrm{Mn}^{\mathrm{II}}$ and $\mathrm{Cu}^{\mathrm{II}}$, moderate quenching was observed for $\mathrm{Fe}^{\mathrm{II}}, \mathrm{Ni}^{\mathrm{II}}$, and $\mathrm{Co}^{\mathrm{II}}$, while the diamagnetic ions $\left(\mathrm{Zn}^{\mathrm{II}}, \mathrm{Mg}^{\mathrm{II}}\right.$, and $\left.\mathrm{Cd}^{\mathrm{II}}\right)$ did not influence the fluorescence intensity. From the Stern-Völmer plots, $K_{\mathrm{sv}}$ values obtained are $(0.91 \pm 0.04) \times 10^{6} \mathrm{M}^{-1}$ and $(0.47 \pm$ 0.02) $\times 10^{6} \mathrm{M}^{-1}$ for $\mathrm{Mn}^{\mathrm{II}}$ and $\mathrm{Cu}^{\mathrm{II}}$, respectively. Although the author stated that the detection limit of $\mathrm{Mn}^{\mathrm{II}}$ was found to be below $0.5 \mathrm{ppb}$, we believe that the low selectivity toward $\mathrm{Mn}^{\mathrm{II}}$ inhibits 30 from use as a sensor in practical applications.

Paramagnetic cations can also quench the luminescence originating from the lanthanide ions; therefore, lanthanide-based MOFs can be also used as "turn-off" sensors for these cations. Zhao et al. reported a luminescent Tb-based MOF (31) synthesized from the use of biphenyltetracarboxylate $\left(\mathrm{bptc}^{4-}\right)$ for selective sensing of $\mathrm{Fe}^{\mathrm{III}}$ [158]. The framework 31 exhibits emission peaks characterized for $\mathrm{Tb}^{\mathrm{III}}$ when it is excited at $310 \mathrm{~nm}$ at room temperature. Suspensions of $\mathbf{3 1}$ in different organic solvents were also subjected to luminescence measurements and the obtained results indicated that their luminescence intensities are solvent-dependent, with the strongest emission observed in ethanol solution. When 31 was added into the ethanol solutions containing different metal ions including $\mathrm{Li}^{\mathrm{I}}$, $\mathrm{Mg}^{\mathrm{II}}, \mathrm{Ca}^{\mathrm{II}}, \mathrm{Co}^{\mathrm{II}}, \mathrm{Ni}^{\mathrm{II}}, \mathrm{Zn}^{\mathrm{II}}, \mathrm{Ag}^{\mathrm{I}}, \mathrm{Cd}^{\mathrm{II}}, \mathrm{Mn}^{\mathrm{II}}, \mathrm{Cr}^{\mathrm{III}}, \mathrm{Al}^{\mathrm{III}}, \mathrm{Cu}^{\mathrm{II}}$, and $\mathrm{Fe}^{\mathrm{III}}$, the fluorescence emission was significantly quenched in the $\mathrm{Fe}^{\mathrm{III}}$ suspension while only a moderate intensity reduction was found in the suspensions containing $\mathrm{Cr}^{\mathrm{III}}, \mathrm{Al}^{\mathrm{III}}$ and $\mathrm{Cu}^{\mathrm{II}}$, and almost no intensity change 
was observed in the case of the other metal ions. The high selectivity of $\mathbf{3 1}$ for Fe $\mathrm{FII}^{\mathrm{III}}$ was attributed to the significant overlap of the d-d absorption band and the excitation spectrum that induces the competitive absorption of excitation energy between $\mathrm{Fe}^{\mathrm{III}}$ and $\mathbf{3 1}$, leading to quenching of the luminescence of $\mathrm{Tb}^{\mathrm{III}}$. The detection of limit was calculated to be 0.1801 mmol L-1 $\left(72.76 \mathrm{ppm}\right.$ ) indicating the relatively high sensitivity of $\mathbf{3 1}$ towards $\mathrm{Fe}^{\mathrm{III}}$ (Figure 18).

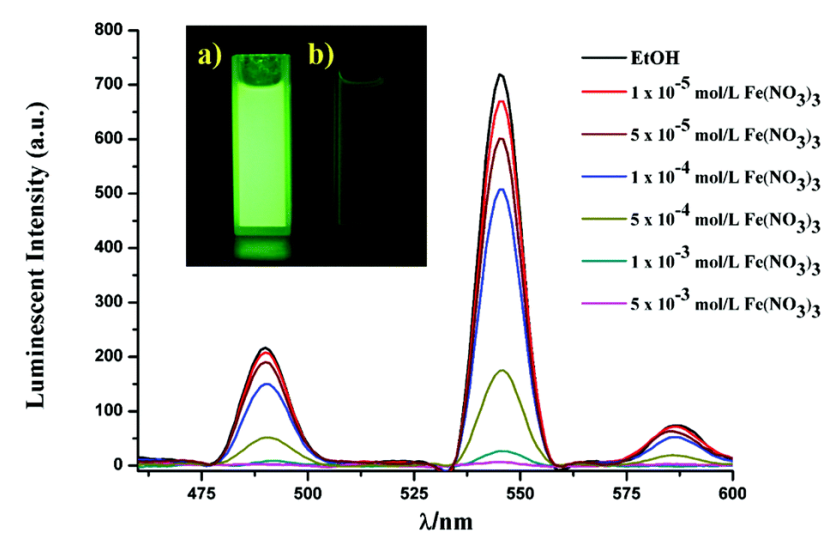

Figure 18. Variation of luminescence intensity of $\mathbf{3 1}$ suspensions with different concentrations of $\mathrm{Fe}\left(\mathrm{NO}_{3}\right)_{3}\left(\lambda_{\mathrm{ex}}=310 \mathrm{~nm}\right)$. Insets: (a) suspension without any metal ions, (b) luminescence quenching after the addition of $\mathrm{Fe}^{\mathrm{III}}$. Reproduced with permission from reference [158].

Recently, Yan et al. also reported two isoreticular lanthanide-based MOFs (32-Eu and 32Tb) for sensing of $\mathrm{Fe}^{\mathrm{III}}$ [159]. Both MOFs were synthesized based on 2,5- di(pyridin-4yl)terephthalic acid $\left(\mathrm{H}_{2} \mathrm{~L}\right)$ and biphenyl-4,4'- dicarboxylic acid $\left(\mathrm{H}_{2} \mathrm{BPDC}\right)$. Under excitation by UV light, both MOFs exhibit characteristic luminescent emissions contributed from the f-f transitions of $\mathrm{Eu}^{\mathrm{III}}$ and $\mathrm{Tb}^{\mathrm{III}}$. The pyridine groups of $\mathrm{H}_{2} \mathrm{~L}$ are uncoordinated and play the role as the binding site for the metal analytes. When different DMF solutions containing $\mathrm{M}\left(\mathrm{NO}_{3}\right)_{\mathrm{x}}$ 
$\left(\mathrm{M}=\mathrm{Na}^{\mathrm{I}}, \mathrm{K}^{\mathrm{I}}, \mathrm{Cu}^{\mathrm{II}}, \mathrm{Fe}^{\mathrm{III}}, \mathrm{Al}^{\mathrm{III}}, \mathrm{Mg}^{\mathrm{II}}, \mathrm{Cr}^{\mathrm{III}}, \mathrm{Zn}^{\mathrm{II}}, \mathrm{Co}^{\mathrm{II}}\right)$ were added in the suspension of crystals of 32, the $\mathrm{Fe}^{\mathrm{III}}$ and $\mathrm{Cu}^{\mathrm{II}}$ ions quench the luminescence of both MOFs, with a larger effect observed with the former. The Stern-Völmer $K_{\mathrm{sv}}$ values obtained at low concentrations of $\mathrm{Fe}^{\mathrm{III}}$ are modest, of $5.16 \times 10^{4} \mathrm{M}^{-1}$ for 32-Eu and $4.30 \times 10^{4} \mathrm{M}^{-1}$ for 32-Tb, respectively.

Encapsulation of luminescent nanoparticles within a non-luminescent MOF is another strategy to obtain a material that can be used for luminescence sensing. Ma et al. recently reported a material (33) formed by simultaneous encapsulation of quantum dots and carbon dots in the well-known ZIF-8 MOF for the detection of $\mathrm{Cu}^{\mathrm{II}}$. The dispersed sample of $\mathbf{3 3}$ in water exhibits orange luminescence, with emission peaks at 430 and $620 \mathrm{~nm}$ upon excitation at $370 \mathrm{~nm}$. When $\mathrm{Cu}^{\mathrm{II}}$ is added to the suspension of $\mathbf{3 3}$, the luminescence is changed from orange to purple due to the quenching of the $620 \mathrm{~nm}$ emission while the luminescence at 430 $\mathrm{nm}$ remains unchanged. Other cations including $\mathrm{Na}^{\mathrm{I}}, \mathrm{K}^{\mathrm{I}}, \mathrm{Cd}^{\mathrm{II}}, \mathrm{Mn}^{\mathrm{II}}, \mathrm{Pb}^{\mathrm{II}}, \mathrm{Al}^{\mathrm{III}}, \mathrm{Zn}^{\mathrm{II}}, \mathrm{Ca}^{\mathrm{II}}$, $\mathrm{Mg}^{\mathrm{II}}, \mathrm{Ag}^{\mathrm{I}}, \mathrm{Fe}^{\mathrm{III}}, \mathrm{Fe}^{\mathrm{II}}, \mathrm{Hg}^{\mathrm{II}}$, common anions, reactive nitrogen species (RNS), reactive oxygen species (ROS), thiols and aminoacids do not interfere the luminescence of $\mathbf{3 3}$, suggesting the high selectivity of this material toward $\mathrm{Cu}^{\mathrm{II}}$. The limit of detection was calculated to be 1.53 $\mathrm{nM}$.

Lu et al. reported a nanoscale MOF (34, also called MOF-253) as a sensor for $\mathrm{Fe}^{\mathrm{II}}$ in both aqueous solution and living cells [161]. 34 is a one-dimensional infinite chain of $\mathrm{AlO}_{6}$ corner-sharing octahedra built by connecting the 2,2'-bipyridine-based bpydc ${ }^{2-}$ linkers to construct rhombic shaped pores. By adding sodium acetate into the reaction, nanoscale powder of 34 was synthesized, with a size of $\sim 50 \mathrm{~nm}$. Upon excitation at $390 \mathrm{~nm}, \mathbf{3 4}$ displays a broad band emission centered at $545 \mathrm{~nm}$, with a quantum yield of $31 \%$ and a luminescence lifetime of $8.19 \mu \mathrm{s}$. When the dehydrated powder of 34 was immersed in an 
aqueous solution containing $\mathrm{Na}^{\mathrm{I}}, \mathrm{Ba}^{\mathrm{II}}, \mathrm{Ca}^{\mathrm{II}}, \mathrm{Mg}^{\mathrm{II}}, \mathrm{Pd}^{\mathrm{II}}, \mathrm{Zn}^{\mathrm{II}}, \mathrm{Cd}^{\mathrm{II}}, \mathrm{Ni}^{\mathrm{II}}, \mathrm{Co}^{\mathrm{II}}, \mathrm{Cu}^{\mathrm{II}}, \mathrm{Cr}^{\mathrm{III}}, \mathrm{Fe}^{\mathrm{II}}$, and $\mathrm{Fe}^{\mathrm{III}}$, only $\mathrm{Fe}^{\mathrm{II}}$ has a significant quenching effect on the fluorescence of 34, which was attributed to the PeT mechanism due to the selective binding of $\mathrm{Fe}^{\mathrm{II}}$ to bipyridine. $\mathbf{3 4}$ was also employed for sensing of $\mathrm{Fe}^{\mathrm{II}}$ in living $\mathrm{HeLa}$ cells, in which significant fluorescence quenching from the intracellular region was observed. When the cells were supplemented with $\mathrm{Fe}^{\mathrm{III}}$ and treated under the same experimental conditions, no fluorescence quenching was observed indicating the high selectivity of $\mathbf{3 4}$ as a sensor for $\mathrm{Fe}^{\mathrm{II}}$.

\subsection{Diamagnetic ions}

Detection of diamagnetic heavy toxic metals such as $\mathrm{Hg}^{\mathrm{II}}$ and $\mathrm{Pb}^{\mathrm{II}}$ in water sources is important because their presence not only contaminates the drinking water supplies but also affects plants and animals. Generally, diamagnetic metal ions do display a large quenching effect of luminescence compared to paramagnetic ones; therefore the sensors can be designed to achieve either "turn-off" or "turn-on" of luminescence. For example, Rudd et al. designed a luminescent Zn-based MOF (35, also called LMOF-263) by incorporating two ligands, one of them is fluorescent (tppe) while the other one provides the binding functional groups $\left(\mathrm{dbtdcO}_{2}{ }^{2-}\right)$ (Figure 19) [162]. The framework 35 displays emission at $464 \mathrm{~nm}$ when excited by UV light $\left(\lambda_{\mathrm{ex}}=365 \mathrm{~nm}\right)$. Upon addition of different metal cations including $\mathrm{Ca}^{\mathrm{II}}, \mathrm{Mg}^{\mathrm{II}}$, $\mathrm{Hg}^{\mathrm{II}}$, and $\mathrm{Pb}^{\mathrm{II}}$ into the aqueous suspension of $\mathbf{3 5}$, the emission of the MOF was quenched to varying degrees. While the influence of $\mathrm{Ca}^{\mathrm{II}}$ and $\mathrm{Mg}^{\mathrm{II}}$ on the fluorescence intensity is negligible, adding of $\mathrm{Hg}^{\mathrm{II}}$ and $\mathrm{Pb}^{\mathrm{II}}$ led to significant quenching, as $84 \%$ and $64 \%$ of the total intensity of $\mathbf{3 5}$ was quenched when its suspension was added $19.6 \mu \mathrm{M}$ of $\mathrm{Hg}^{\mathrm{II}}$ and $\mathrm{Pb}^{\mathrm{II}}$, respectively. Although the mechanism of this quenching effect is unclear, the authors attributed this phenomenon to the interaction between the metal ions and the sulfone group. Nevertheless, $\mathbf{3 5}$ is a promising chemical sensor for $\mathrm{Hg}^{\mathrm{II}}$ and $\mathrm{Pb}^{\mathrm{II}}$, as the $\mathrm{K}_{\mathrm{SV}}$ constants were 
determined to be 459446 and $55017 \mathrm{M}^{-1}$, and the detection limits were 3.3 and $19.7 \mathrm{ppb}$ for $\mathrm{Hg}^{\mathrm{II}}$ and $\mathrm{Pb}^{\mathrm{II}}$, respectively.

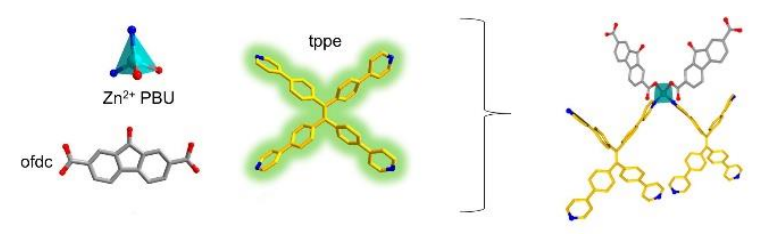

Figure 19. Structure of $\mathbf{3 5}$, formed by employing both the luminescent tppe ligand and the $\mathrm{dbtdcO}_{2}{ }^{2-}$ ligand providing the binding sites for metal analytes. Reproduced with permission from reference [162].

Lim et al. reported a Zn-based MOF (36) as a turn-on sensor for $\mathrm{Cd}^{\mathrm{II}}$, which is another common toxic metal ion in water [163]. The framework 36 was synthesized from a solvothermal reaction using 4',4"',4"'"-nitrilotris(3-methoxy-[1,1'-biphenyl]-4-carboxylic acid $\left(\mathrm{H}_{3} \mathrm{~L}\right)$. The MOF exhibits weak ligand-based fluorescent emission at $482 \mathrm{~nm}$ when excited at $375 \mathrm{~nm}$. When different metal ions including $\mathrm{Na}^{\mathrm{I}}, \mathrm{Zn}^{\mathrm{II}}, \mathrm{Ca}^{\mathrm{II}}, \mathrm{K}^{\mathrm{I}}, \mathrm{Ag}^{\mathrm{I}}, \mathrm{Pb}^{\mathrm{II}}, \mathrm{Hg}^{\mathrm{II}}$, $\mathrm{Mn}^{\mathrm{II}}, \mathrm{Ni}^{\mathrm{II}}, \mathrm{Mg}^{\mathrm{II}}, \mathrm{Co}^{\mathrm{II}}, \mathrm{Cu}^{\mathrm{II}}$ and $\mathrm{Cd}^{\mathrm{II}}$ were added to suspensions of $\mathbf{3 6}$ in DMF, only in the case of $\mathrm{Cd}^{\mathrm{II}}$ the fluorescence intensity was enhanced. Increasing the concentration of $\mathrm{Cd}^{\mathrm{II}}$ from 0 to $5000 \mathrm{ppm}$ leads to the increase of the emission signal, with the intensity (I) linearly proportional to the concentration $(C)$ of $\mathrm{Cd}^{\mathrm{II}}$ in $\mathrm{ppm}, \mathrm{I}=146.2+1.47 \times \mathrm{C}$. The mechanism of the fluorescence enhancing is not very clear although the authors suggested that the interaction between the $\mathrm{N}$ atom from $\mathrm{L}^{3-}$ and $\mathrm{Cd}^{\mathrm{II}}$ is responsible for the observed enhancing behavior. The $\mathrm{Cd}^{\mathrm{II}}$ in the pores of the MOF can be washed with DMF to restore the MOF to its pristine state, suggesting the reversibility of $\mathbf{3 5}$ for sensing application.

Chen et al. reported a rare example of a Co-based MOF (37) that exhibits a very selective sensing towards $\mathrm{Al}^{\mathrm{III}}$ [164]. Sensing of $\mathrm{Al}^{\mathrm{III}}$ is important as chronic intoxication of this ion in 
the body produces neuropathological hallmarks of Alzheimer disease [185]. The MOF 37 was formed based on the solvothermal reaction of 4,6-di(2-methyl-imidazol-1-yl)-pyrimidine (dmimpym) and 1,4-naphthalenedicarboxylic acid $\left(\mathrm{H}_{2}\right.$ nda $)$ with $\mathrm{Co}\left(\mathrm{NO}_{3}\right)_{2} \cdot 6 \mathrm{H}_{2} \mathrm{O}$. The dmimpym ligand has uncoordinated pyrimidyl sites that are responsible for the binding with metal analytes (Figure 20, left). Although 37 is based on $\mathrm{Co}^{\mathrm{II}}$ ion, which is paramagnetic, the MOF still displays emission at $398 \mathrm{~nm}$, which according to the authors was attributed to the mixed contribution of the ILCT and LLCT ( $\pi^{*} \rightarrow \pi$ and $\pi^{*} \rightarrow \mathrm{n}$ transitions) of the two conjugated ligands. When $5 \mathrm{mg}$ ground powder of $\mathbf{3 7}$ was immersed in $5 \mathrm{~mL}$ of DMF and this solution was titrated by adding an aqueous solution containing different metal ions $\left(\mathrm{Na}^{\mathrm{I}}, \mathrm{K}^{\mathrm{I}}, \mathrm{Ag}^{\mathrm{I}}, \mathrm{Mg}^{\mathrm{II}}, \mathrm{Ba}^{\mathrm{II}}, \mathrm{Ca}^{\mathrm{II}}, \mathrm{Pb}^{\mathrm{II}}, \mathrm{Ni}^{\mathrm{II}}, \mathrm{Cd}^{\mathrm{II}}, \mathrm{Zn}^{\mathrm{II}}\right.$, $\mathrm{Al}^{\mathrm{III}}, \mathrm{Cr}^{\mathrm{III}}, \mathrm{Hg}^{\mathrm{II}}, \mathrm{Cu}^{\mathrm{II}}$, and $\left.\mathrm{Fe}^{\mathrm{III}}\right)$, only $\mathrm{Al}^{\mathrm{III}}$ displayed enhanced luminescence. Similar to the example above, the mechanism of this luminescent enhanced effect is unclear to us; nevertheless, this suggests the high selectivity of $\mathbf{3 7}$ towards $\mathrm{Al}^{\mathrm{III}}$. The LOD was calculated to be as low as 1.40 $\mu \mathrm{M}$, which is equivalent to $35.4 \mathrm{ppb}$ of $\mathrm{Al}^{\mathrm{III}}$ ions in an aqueous medium. Framework 37 can be recycled by washing with DMF and the obtained solid was demonstrated to exhibit similar emissions after at least 5 cycles.

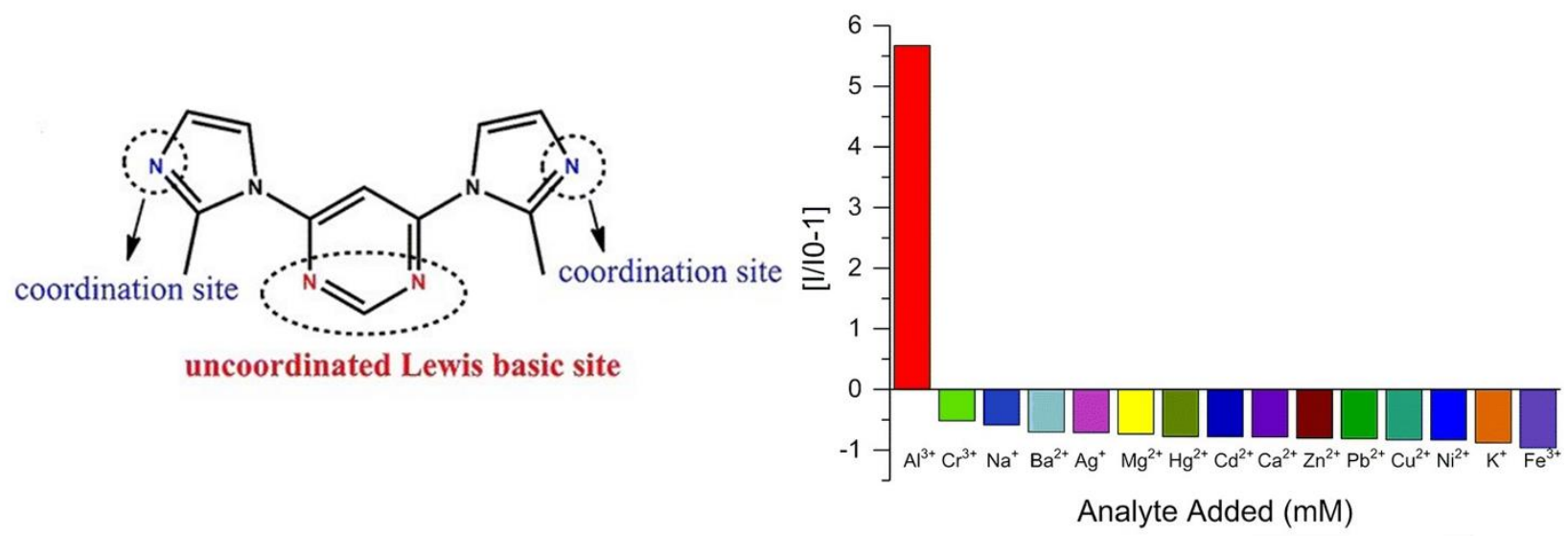


Figure 20. (Left) The structure of dmimpym. (Right) Changes in the luminescence intensity with respect to the emission of $\mathbf{3 7}$ (at $407 \mathrm{~nm}$ ) with $50 \mu \mathrm{L}$ of an aqueous solution containing $10 \mathrm{mM}$ different metal ions. Reproduced with permission from reference [164].

While the enhancement or quenching of luminescence is often unpredictable for ligand-based luminescent MOFs, the luminescence of lanthanide ions is more controllable. For example, one way to obtain the luminescence enhancement of lanthanide ions is improving the intersystem crossing using the heavy atom effect. A typical example was the work by Hao et al., who reported an Al-based Eu-doped MOF (38, also called EuIII@MIL-121) synthesized from the use of benzenetetracarboxylic acid for sensing of $\mathrm{Ag}^{\mathrm{I}}$ [165]. Framework 38 exhibits characteristic emission of Eu ${ }^{\mathrm{III}}$ under $\mathrm{UV}$-light radiation at $254 \mathrm{~nm}$, with a lifetime of $0.31 \mathrm{~ms}$ and a quantum yield of $6 \%$. When the as-made samples of 37 were immersed in aqueous solutions containing different metal ions including $\mathrm{Na}^{\mathrm{I}}, \mathrm{K}^{\mathrm{I}}, \mathrm{Mg}^{\mathrm{II}}, \mathrm{Ca}^{\mathrm{II}}, \mathrm{Al}^{\mathrm{III}}, \mathrm{Cr}^{\mathrm{III}}, \mathrm{Mn}^{\mathrm{II}}, \mathrm{Fe}^{\mathrm{III}}$, $\mathrm{Co}^{\mathrm{II}}, \mathrm{Ni}^{\mathrm{II}}, \mathrm{Cu}^{\mathrm{II}}, \mathrm{Ag}^{\mathrm{I}}, \mathrm{Zn}^{\mathrm{II}}, \mathrm{Cd}^{\mathrm{II}}, \mathrm{Hg}^{\mathrm{II}}$, and $\mathrm{Pb}^{\mathrm{II}}$, only the MOF suspension with $\mathrm{Ag}^{\mathrm{I}}$ exhibited visible red-color luminescence (Figure 21). The enhancement of luminescence intensity by $\mathrm{Ag}^{\mathrm{I}}$ was attributed to the heavy-atom effect that leads to more efficient energy transfer from ligands to $\mathrm{Eu}^{\mathrm{III}}$ ions. The detection limit of $\mathbf{3 8}$ towards $\mathrm{Ag}^{\mathrm{I}}$ was found to be $0.1 \mathrm{mM}$, which met the $50 \mu \mathrm{g} \mathrm{L}^{-1}(\sim 0.46 \mathrm{mM})$ standard of U.S. Environmental Protection Agency (EPA) for a maximum allowable level of $\mathrm{Ag}^{\mathrm{I}}$ in drinking water [186].

\subsection{Sensing of anions}

Similar to heavy metal cations, several anions such as $\mathrm{CrO}_{4}{ }^{2-}$ and $\mathrm{Cr}_{2} \mathrm{O}_{7}{ }^{2-}$ are highly toxic and can accumulate in living organisms leading to cancer, deformity, or even gene mutation, which is a significant threat to the environment [187]. Recently, Yi et al. reported three Cdbased MOFs (39-1, 39-2, 39-3) for sensing of $\mathrm{CrO}_{4}{ }^{2-}$ and $\mathrm{Cr}_{2} \mathrm{O}_{7}{ }^{2-}$ [166]. The MOFs are 
formed from the use of a hexacarboxylate ligand hexa[4-(carboxyphenyl)oxamethyl]-3oxapentane $\left(\mathrm{L}^{6-}\right)$ and one of the space-directed auxiliary ligands including 2,2'-bipyridine (bpy), 4,4'- di(1H-imidazol-1-yl)-1,1'-biphenyl (bib), and 1,3,5-tri(1H-imidazol-1-yl)benzene (tib). All the MOFs exhibit luminescent emission, with their spectra similar to those of the Ndonor ligands. When the as-synthesized samples of 39-1, 39-2, and 39-3 were ground and suspended in an aqueous solution $\left(0.01 \mathrm{~mol} \mathrm{~L}^{-1}\right)$ containing a variety of different anions including $\mathrm{F}^{-}, \mathrm{Cl}^{-}, \mathrm{Br}^{-}, \mathrm{N}_{3}^{-}, \mathrm{IO}_{3}^{-}, \mathrm{BrO}_{3}{ }^{-}, \mathrm{AcO}^{-}, \mathrm{CO}_{3}{ }^{2-}, \mathrm{SO}_{4}{ }^{2-}, \mathrm{CrO}_{4}{ }^{2-}, \mathrm{Cr}_{2} \mathrm{O}_{7}{ }^{2-}, \mathrm{MoO}_{4}{ }^{2-}, \mathrm{WO}_{4}{ }^{2-}$, and $\mathrm{PO}_{4}{ }^{3-}$, it was found that the $\mathrm{CrO}_{4}{ }^{2-}$ and $\mathrm{Cr}_{2} \mathrm{O}_{7}{ }^{2-}$ ions afforded the most significant quenching effect, which occurred instantly and reaches $100 \%$ turn-off within 5 minutes. The other anions do not or only slightly quench the MOFs' luminescence intensity. The luminescence quenching phenomenon by $\mathrm{CrO}_{4}{ }^{2-}$ and $\mathrm{Cr}_{2} \mathrm{O}_{7}{ }^{2-}$ ions was attributed to the overlap of their light absorption bands (230 - $413 \mathrm{~nm})$ with the ones of the MOFs, which makes them different from the other anions (Figure 21). Upon illumination, the absorption of $\mathrm{CrO}_{4}{ }^{2-}$ and $\mathrm{Cr}_{2} \mathrm{O}_{7}{ }^{2-}$ hinders the absorption of the MOFs, thus resulting in a decrease, or even full quenching of the luminescence intensities. This mechanism of the quenching effect seems universal and works on other luminescent MOFs as well, with similar reports have recently been noticed [188].

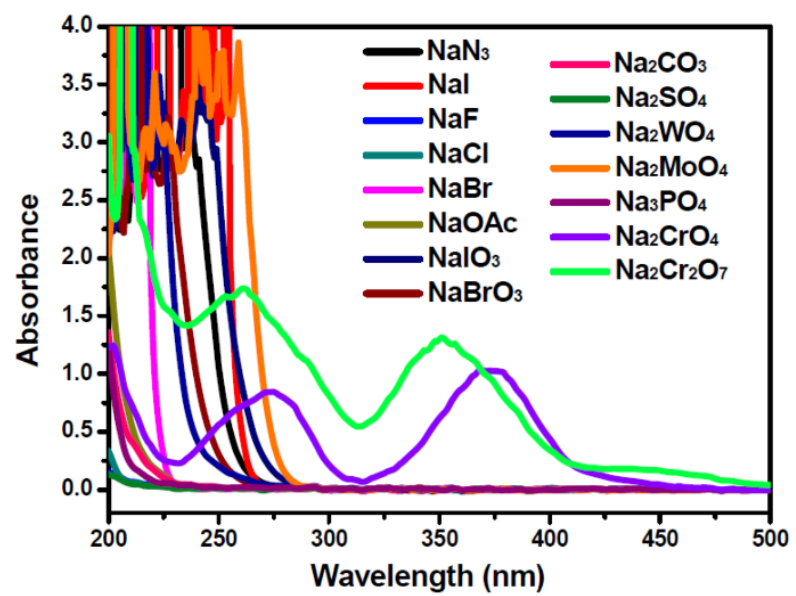


Figure 21. UV-vis spectra of various anions in the aqueous solution. Reproduced with permission from reference [166].

Zhou et al. also reported a luminescent Tb-based MOF (40) for the detection of $\mathrm{CrO}_{4}{ }^{2-}$ in aqueous solution [167]. The MOF was formed from the use of both 2,2-dipyridine-3,3'dicarboxylate $\left(\mathrm{BPDC}^{2-}\right)$ and 1,1'-biphenyl-4,4'-dicarboxylate $\left(\mathrm{BPDCA}^{2-}\right)$ as the organic ligands, and is a cationic MOF with $\mathrm{ClO}_{4}{ }^{-}$being the counter anion. The desolvated sample of 40 exhibits characteristic emission bands of $\mathrm{Tb}^{\mathrm{III}}$ ion when it is excited at $300 \mathrm{~nm}$. The anion $\mathrm{ClO}_{4}^{-}$present in $\mathbf{4 0}$ can be exchanged by other anions, which might affect the luminescence intensity of the MOF. When titrated with various anions including $\mathrm{F}^{-}, \mathrm{Cl}^{-}, \mathrm{Br}^{-}, \mathrm{I}^{-}, \mathrm{SO}_{4}{ }^{2-}$, $\mathrm{CO}_{3}{ }^{2-}, \mathrm{NO}_{3}{ }^{-}, \mathrm{CrO}_{4}{ }^{2-}$, and $\mathrm{OAc}^{-}, 40$ displays the largest quenching effect with $\mathrm{CrO}_{4}{ }^{2-}$, which was partially attributed to the light absorption of anion itself and the weak interaction with the framework that may influence the efficiency of the energy transfer process.

Utilizing the same strategy of anion exchange, Shi et al. reported a cationic MOF (41) for sensing of $\mathrm{I}^{-}$in aqueous solution [168]. Framework 41 is composed of [ $\mathrm{Tb}_{2}$ ] units connected together by $\left[\mathrm{Zn}(\mathrm{L})_{3}\right]$ ligands $\left(\mathrm{H}_{2} \mathrm{~L}=2,2^{\prime}\right.$-dipyridine-4,4'-dicarboxylic acid $)$ into a 3dimensional network, with $\mathrm{NO}_{3}^{-}$being the counter anion. The aqueous suspension of the MOF exhibits characteristic emission bands of $\mathrm{Tb}^{\mathrm{III}}$ when excited at $343 \mathrm{~nm}$. The $\mathrm{NO}_{3}{ }^{-}$ counter anions in the void channel can be readily exchanged with other anions. When common anions including $\mathrm{F}^{-}, \mathrm{Cl}^{-}, \mathrm{Br}^{-}, \mathrm{I}^{-}, \mathrm{SCN}^{-}, \mathrm{N}_{3}^{-}, \mathrm{AcO}^{-}, \mathrm{NO}_{3}^{-}, \mathrm{H}_{2} \mathrm{PO}_{4}^{-}, \mathrm{HSO}_{3}^{-}, \mathrm{HCO}_{3}^{-}$, $\mathrm{CO}_{3}{ }^{2-}, \mathrm{SO}_{4}{ }^{2-}, \mathrm{SO}_{3}{ }^{2-}$, and $\mathrm{PO}_{4}{ }^{3-}$ were added to aqueous suspensions of $\mathbf{4 1}$, in the case of $\mathrm{I}^{-}$, the luminescence was almost quenched, with the peaks at 585 and $621 \mathrm{~nm}$ disappeared. This luminescence quenching phenomenon by $\mathrm{I}^{-}$was attributed to the partial oxidation of $\mathrm{I}^{-}$, probably due to the presence of $\mathrm{NO}_{3}{ }^{-}$, to form $\mathrm{I}_{3}{ }^{-}$, which can effectively absorb the energy of 
excitation light and reduce the energy transfer to the $\mathrm{Tb}^{\mathrm{III}}$ ion. By varying the concentration of $\mathrm{I}^{-}$, the detection limit of $\mathrm{I}^{-}$was found to be $1 \times 10^{-8} \mathrm{~mol} \mathrm{~L}^{-1}$. From the Stern- Völmer plot, the $\mathrm{K}_{\mathrm{SV}}$ value of $\mathbf{4 1}$ is $1.8 \times 10^{5} \mathrm{~L} \mathrm{~mol}^{-1}$.

Yang et al. recently reported a boric-acid functionalized lanthanide MOF (42) for sensing of $\mathrm{F}^{-}$anion. $\mathrm{F}^{-}$is a common anion present in ground and drinking water. The presence of $\mathrm{F}^{-}$in high concentrations (1.5 -2 ppm) can have an adverse effect on tooth enamel and may give rise to dental fluorosis. Elevated intake of $\mathrm{F}^{-}(>3 \mathrm{ppm})$ for a long period of time can cause skeletal fluorosis and even crippling skeletal fluorosis. The World Health Organization (WHO) guideline suggests that the concentration of $\mathrm{F}^{-}$in drinking water should be $<1.5 \mathrm{ppm}$ [189]. The MOF 42 is based on $\mathrm{Eu}^{\mathrm{III}}$ and 5-boronoisophthlate (bop ${ }^{2-}$ ) ligand, and exhibits two strong emission bands at 366 and 590-625 nm; the former was assigned to the emission of bop $^{2-}$ while the latter is the characteristic emission of $\mathrm{Eu}^{\mathrm{III}}$. Boronic acid has strong affinity toward $\mathrm{F}^{-}$(Figure 22); when $\mathrm{F}^{-}$is added into the suspension of $\mathbf{4 2}$, the emission at $366 \mathrm{~nm}$ is enhanced while the emission at $\sim 625 \mathrm{~nm}$ decreased gradually with increased $\mathrm{F}^{-}$concentration allowing for the ratiometric sensing of $\mathrm{F}^{-}$. The limit of detection was found of $2 \mu \mathrm{M}$ or 0.034 ppm, which is much lower than the concentration suggested by WHO aforementioned.

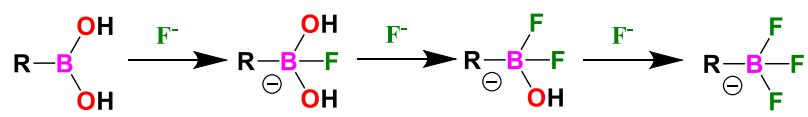

Figure 22. Reaction between boronic acid and $\mathrm{F}^{-}$.

\subsection{Sensing of gases and organic molecules}

Luminescent MOFs for sensing of gases and organic molecules, especially nitroaromatic explosives, were recently reviewed by other authors such as Lin et al. [122], Zhang et al. [190], Zhang et al. [125], Jingrong et al. [191] and Wang et al. [192] among others. In this 
part, we review the very recent interesting articles. Similar to the sensing of cations or anions, MOFs can be designed as a turn-off or turn-on sensors for gas and organic molecules. This partly depends on the characteristics of the analytes, i.e. analytes with strong electron withdrawing groups tend to quench luminescence. For example, Roales et al. reported the preparation of a Zn-based MOF (43) film by soft-imprinting for turn-off sensing of 2,4dinitrotoluene (DNT) gas [170]. DNT is highly toxic since it converts hemoglobin to methemoglobin, which cannot bind and transport oxygen in blood. DNT is also part of the composition of explosives; therefore, sensing DNT has attracted great attention recently for anti-terrorism operations. The MOF 43 was prepared by employing two ligands, 4,4' biphenyldicarboxylate $\left(\mathrm{bpdc}^{2-}\right)$ and 1,2-bipyridylethene (bpee), with both of them acting as the luminescence sources [193]. Upon excitation at $280 \mathrm{~nm}, 43$ exhibits emission at $460 \mathrm{~nm}$. The crystalline powder of $\mathbf{4 3}$ was then incorporated into a cellulose acetate thin film spincoated on quartz. The exposure of the film to DNT vapors resulted in quenching of the luminescence, this is due to the electron withdrawing nature of the $-\mathrm{NO}_{2}$ groups of DNT. The mean fluorescence quenching for a 10 second exposure is $\sim 15 \%$. Although the sensing performance of this film is quite modest, we believe that soft-imprinting of luminescent MOFs is a good strategy for fabricating good gas sensors.

Oxygen $\left(\mathrm{O}_{2}\right)$ is a known quencher of lanthanide luminescence due to the deactivation of the triplet-state of the organic antenna; therefore, Ln-based MOFs are ideal candidates for $\mathrm{O}_{2}$ sensing. One example is the work by Xu et al., who reported a nanoscale Eu MOF (44) synthesized from the solvothermal synthesis between $\mathrm{Eu}\left(\mathrm{NO}_{3}\right)_{3}$ and the luminescent organic linker $\mathrm{H}_{2}$ pbydc for ratiometric sensing of $\mathrm{O}_{2}$ [171]. The MOF nanoparticles were incorporated on the surface of the nonwoven polypropylene (PP) fibers, which were then coated with polydimethylsiloxane (PDMS) to enhance their moisture resistance. Upon 
excitation at $395 \mathrm{~nm}, \mathbf{4 4 @ P P}$ exhibits emission from both the ligand pbydc ${ }^{2-}\left(\lambda_{\mathrm{em}}=557 \mathrm{~nm}\right)$ and $\mathrm{Eu}^{\mathrm{III}}\left(\lambda_{\mathrm{em}}=614 \mathrm{~nm}\right)$. Due to the low energy of the triplet state of pbydc ${ }^{2-}\left(17950 \mathrm{~cm}^{-1}\right)$, which is close to the energy of the emissive state of $\mathrm{Eu}^{\mathrm{III}}\left({ }^{5} \mathrm{D}_{0}, 17250 \mathrm{~cm}^{-1}\right)$, exposure of 44@PP to $\mathrm{O}_{2}$ leads to quenching of the Eu ${ }^{\mathrm{III}}$ luminescence emission at $614 \mathrm{~nm}$, while the emission from the ligand pbydc ${ }^{2-}$ remains unchanged. At 1 atm $\mathrm{O}_{2}$, the emission of $\mathrm{Eu}^{\mathrm{III}}$ at $614 \mathrm{~nm}$ was quenched by $89.9 \%$, and the quenching efficiency $\left(\mathrm{R}_{0} / \mathrm{R}\right)$ is of 7.66 . The LOD of 44@PP was calculated to be $0.45 \%$ of $\mathrm{O}_{2}$ in an $\mathrm{O}_{2} / \mathrm{N}_{2}$ mixture.

Very recently, Zhang et al. reported the use of a Eu MOF film (45) for sensing of sulfur dioxide $\left(\mathrm{SO}_{2}\right)$ gas [172]. The film was fabricated based on an in situ secondary growth method on a glass slide using $\mathrm{UiO}-66-\mathrm{NH}_{2}$ as the seed layer. 45 displays characteristic red emission of $\mathrm{Eu}^{\mathrm{III}}$ at $615 \mathrm{~nm}$ upon excitation at $370 \mathrm{~nm}$. When the film was exposed to different gases including $\mathrm{N}_{2}, \mathrm{CO}_{2}, \mathrm{O}_{2}, \mathrm{NH}_{3}, \mathrm{HCHO}, \mathrm{H}_{2} \mathrm{O}, \mathrm{H}_{2} \mathrm{~S}, \mathrm{NO}_{2}$, and $\mathrm{SO}_{2}$, only $\mathrm{SO}_{2}$ induced significant luminescence quenching, up to $98.33 \%$ at the concentration of $200 \mathrm{ppm}$. This quenching was attributed to the charge transfer between the ligand and $\mathrm{SO}_{2}$ molecules. In the range of 0-25 ppm, the luminescence intensity was linearly decreased with increasing the concentration of $\mathrm{SO}_{2}$. The LOD of the film was estimated to be $0.65 \mathrm{ppm}$. In addition, the film is recyclable after at least 10 cycles, with the luminescence intensity still exhibiting $88.2 \%$ of the initial intensity.

Sensing of volatile organic compounds (VOCs) was recently demonstrated by Huang et al., who prepared the MOF 46 based on linking the $\mathrm{Ag}_{12}$ chalcogenide/chalcogenolate clusters with 4,4'-bipyridine [173]. Different from the $\mathrm{Ag}_{12}$ cluster, whose crystals deteriorate completely in less than 30 min in air, $\mathbf{4 6}$ exhibits much higher stability and its crystals retain their morphology and crystallinity for one year under ambient conditions. $\mathbf{4 6}$ displays yellow 
luminescence in the mother liquor but this emission is significantly quenched in air due to the quenching by $\mathrm{O}_{2}$. In vacuum, the crystals of $\mathbf{4 6}$ emit bright green luminescence $\left(\lambda_{\mathrm{em}}=507\right.$ $\mathrm{nm}$ ), with a quantum yield of $12.1 \%$ and a lifetime of $0.20 \mu$ s. The loss of luminescence of 46 in air can be restored in various VOCs, as shown in Figure 23, which was attributed to the intercalation of VOCs inside the channels of $\mathbf{4 6}$ impeding its contact with $\mathrm{O}_{2}$. Among the VOCs, EtOH enhances the luminescence intensity of $\mathbf{4 6}$ by $\sim 26$-fold relative to air and the response time is less than $1 \mathrm{~s}$. This suggests the potential application of $\mathbf{4 6}$ for sensing trace EtOH in air or in the exhaled breath of individuals who have consumed alcohol.

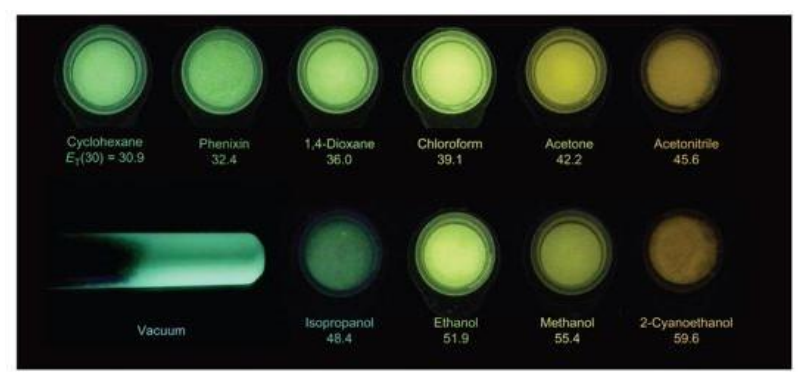

Figure 23. Luminescence responses of $\mathbf{4 6}$ in different VOCs. Reproduced with permission from reference [173].

Li et al. reported a Zn-based MOF (47, also called JLU-MOF48) as a sensor for the detection of nitroaromatic explosives in solution [174]. 47 was synthesized from the solvothermal reaction between $\mathrm{ZnSO}_{4}$ and 2-(3,5-Dicarboxyphenyl)-1 $\mathrm{H}$-imidazole-4,5-dicarboxylic acid $\left(\mathrm{H}_{5} \mathrm{DpImDc}\right)$. The MOF suspension in DMF displays emission peak centered at $366 \mathrm{~nm}$, which is quenched when solutions of nitroaromatic explosives such as 2,4,6-trinitrophenol (TNP), 2,4-dinitrophenol (2,4-DNP), and 4-nitrophenol (4-NP) are added. The fluorescence intensity decreases by $95.7 \%$ for TNP, and the limit of detection of TNP is $0.25 \mathrm{ppm}$. 
Hou et al. also reported a turn-off sensor based on an In MOF (48) for detecting nitrofurazone (NZF) [175]. NZF is a synthetic antibiotic that used to be employed in animal husbandry and aquaculture, but was banned in 2002 by the FDA due to evidence that the drugs may induce carcinogenic residues in animal tissues. However, it still can be found in aquatic products and ground water nowadays; therefore detection of NZF is essential. The MOF 48 was synthesized from a solvothermal reaction between 5-(2,6-bis(4-carboxyphenyl) pyridin-4-yl) isophthalic acid $\left(\mathrm{H}_{4} \mathrm{BCP}\right)$ with $\mathrm{In}\left(\mathrm{NO}_{3}\right)_{3}$ in DEF. Under excitation at $300 \mathrm{~nm}, 48$ exhibits emission at $372 \mathrm{~nm}$, which was attributed to the emission of the ligand $\left(\mathrm{BCP}^{4-}\right)$. When the suspension of crystalline powder of $\mathbf{4 8}$ is treated with an NZF aqueous solution, the luminescence of $\mathbf{4 8}$ is significantly quenched and this decrease in the luminescence intensity still can be seen in a $0.2 \mathrm{ppm}$ NZF solution. Other antibiotics such as sulfadiazine, chloramphenicol, sulfamethazine, thiamphenicol, nitrofurantoin, dimetridazole, ronidazole, ornidazole, metronidazole do not affect the luminescence of 48 suggesting the high selectivity of this MOF towards NZF.

Yue et al. reported a Ce-based MOF (49) for turn-on sensing of ascorbic acid (AA, vitamin C) [176], of which the determination is significant in disease diagnosis and food safety in daily life. Framework 49 was synthesized using 1,1':4',1"-terphenyl-2',4,4",5'-tetracarboxylic acid; the Ce ions have mixed valence state: $+3(40 \%)$ and $+4(60 \%)$. The emission spectrum of 49 shows an intense broad band with a maximum at $380 \mathrm{~nm}$ upon excitation at $316 \mathrm{~nm}$. When titrated with AA, the fluorescence intensity of $\mathbf{4 9}$ was enhanced and the emission band was slightly red-shifted with increasing the concentration of AA. The author explained this phenomenon through the redox reaction between $\mathrm{Ce}^{\mathrm{IV}}$ and $\mathrm{AA}$, after which $\mathrm{AA}$ was oxidized to generate dehydroascorbic acid (DHA) (Figure 24). The DHA molecule has three electronwithdrawing ketone groups and hence blocks the photoinduced electron transfer process from 
the TPTC ligand to the $\mathrm{Ce}^{\mathrm{IV}}$ ion. Consequently, the fluorescence of the TPTC ligand is enhanced. The detection limit was estimated to be $7 \mathrm{nM}$, which is quite low, suggesting that 49 is a promising material for AA detection in practical applications.

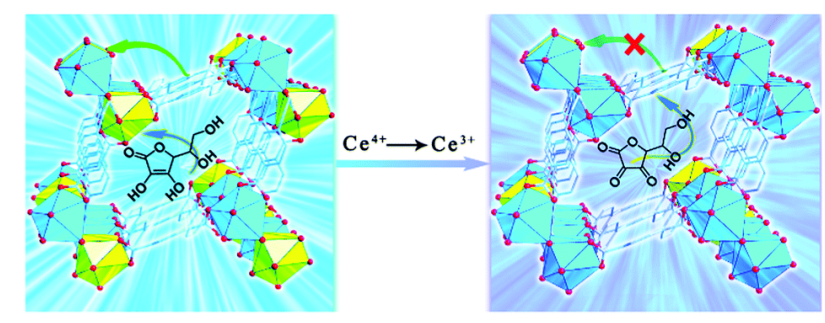

Figure 24. Schematic demonstration of the detection of AA by 49. Reproduced with permission from reference [176].

Gao et al. recently reported a mixed lanthanide-based MOF (50) for the recognition and detection of pharmaceuticals. The MOF is based on a mixture of Eu and Tb (ratio of $0.1: 0.9$ ) with $\mathrm{BTC}^{3-}$ being the ligand. The crystals of $\mathbf{5 0}$ were coated on an indium tin oxide (ITO) glass to form a thin film. When the film was placed in aqueous solutions of different pharmaceuticals including antipyrine, benzafibrate, caffeine, clofibrate, clotetracycline, coumarin, diclofenac, fluorouracil, nalidixic acid, naproxen, sulfachinoxalin, and tetracycline, it was found that the emission intensity of the film was dependent on individual pharmaceutical compounds suggesting discrimination between them based on the value of the intensity ratio change (Figure 25, top). A consequence of this phenomenon is that the films exhibit different colors depending on the analytes (Figure 25, bottom). This phenomenon was attributed to the different functional groups and structures of pharmaceutical molecules generate different hydrogen-bonding and/or hydrophobic interactions, leading to the different energy transfer mechanisms. 

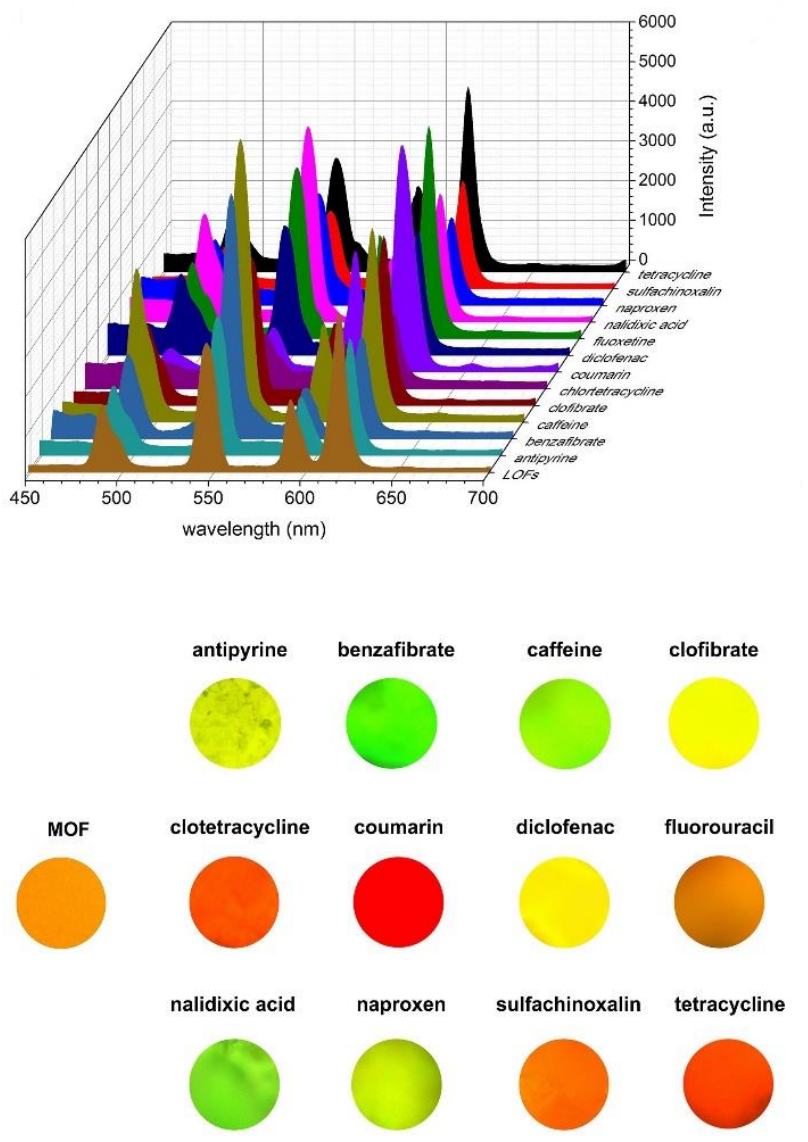

Figure 25. The emission spectra (top) and the optical photographs (bottom) of thin films of $\mathbf{5 0}$ in the presence of different pharmaceutical analytes. Reproduced with permission from reference [177].

\section{Anti-Stokes Shift Luminescent Metal-organic Frameworks}

The luminescent MOFs that have thus far been discussed in this review emit lower energy photons than their excitation sources, with a large Stokes shift being helpful to clearly separate excitation and emission signals and to prevent self-quenching. In this section, we discuss luminescent MOFs that emit photons at higher energies than those used for excitation, i.e. MOFs exhibiting anti-Stokes shift luminescence. This type of emission from materials can be observed in two broad cases, either when certain types of materials, exposed to highintensity laser radiation, exhibit non-linear optical (NLO) effects, or when the absorption of 
low-energy photons populates higher-lying energy levels within a material, from which radiative transitions can occur, i.e. upconversion of luminescence (UCL). In this review, we focus on the latter type of anti-Stokes luminescence, i.e. UCL. For the sake of completeness however, and to place the phenomenon of UCL into context, we first introduce some elementary concepts of NLO, as well as a few of the earliest examples of NLO MOFs. For deeper insight into NLO-active MOFs, the reader is directed to several comprehensive reviews addressing the subject. As the terminology used in the field may vary from author to author, we begin this section by first underlining the differences between NLO and UCL, defining the key physical phenomena related to both, and then detailing examples of MOFs that exhibit them. The list of MOFs reviewed in this section is provided in Table 2.

Table 2. Structural information and photophysical properties of luminescent MOFs for NLO and UCL.

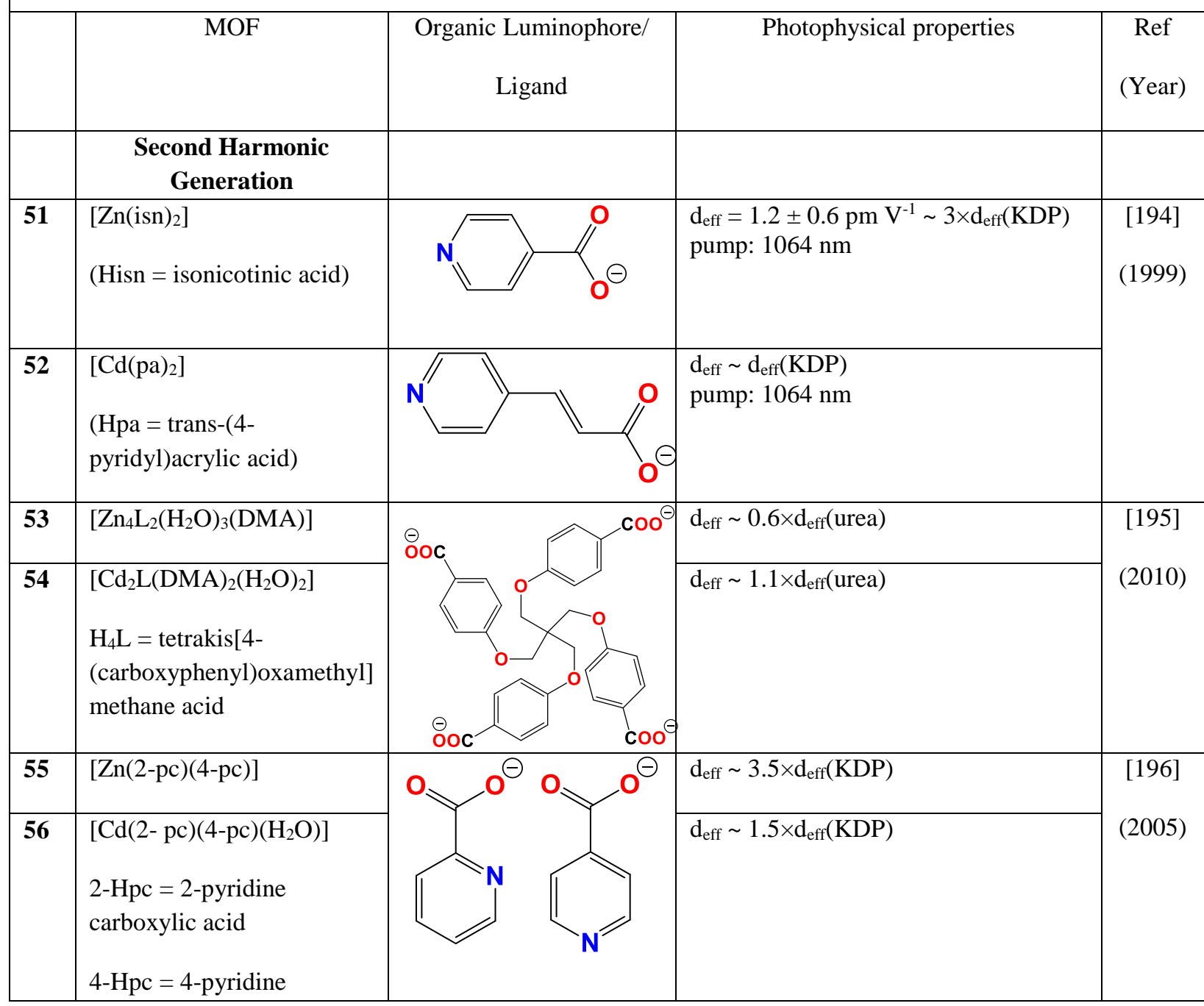




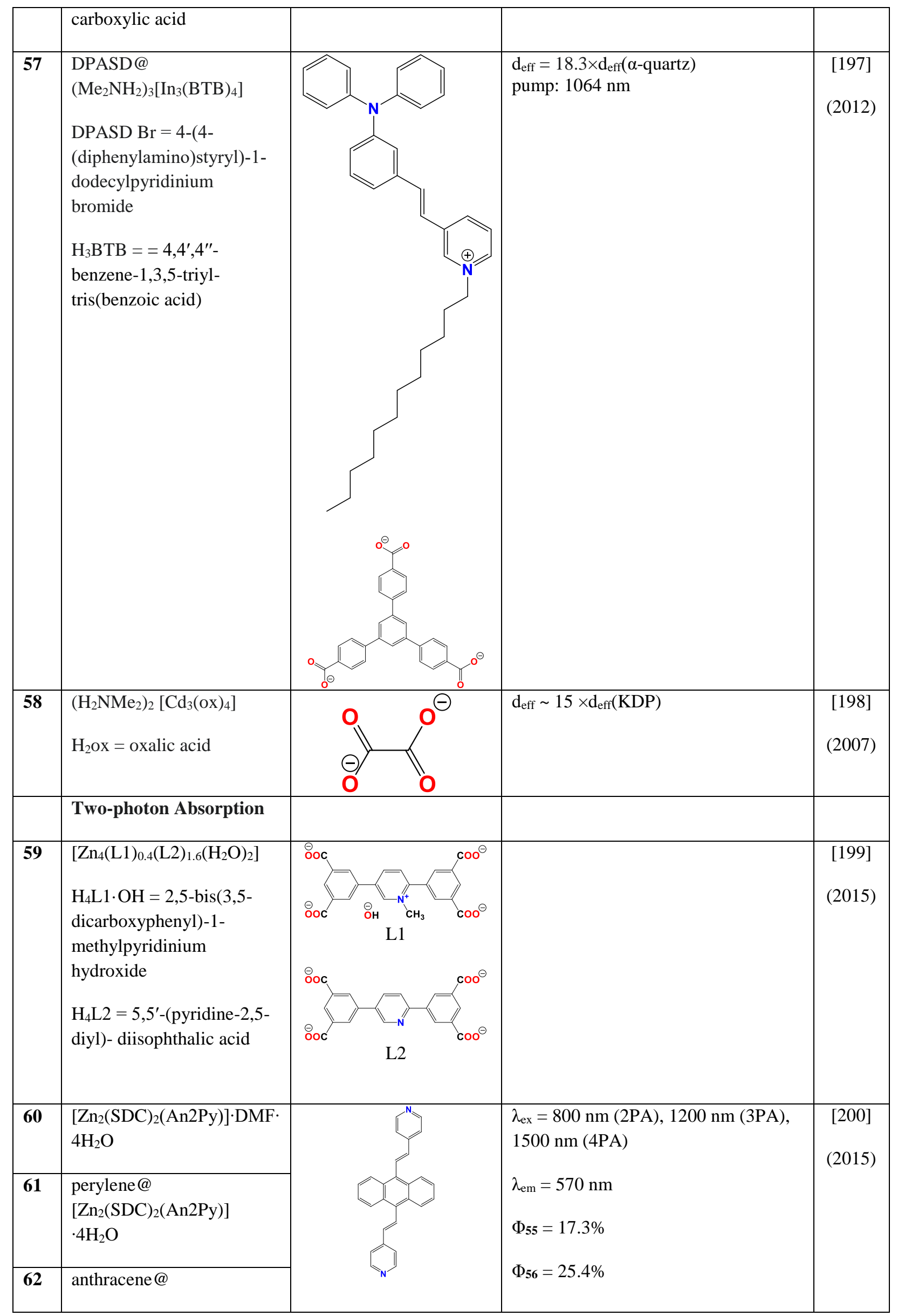




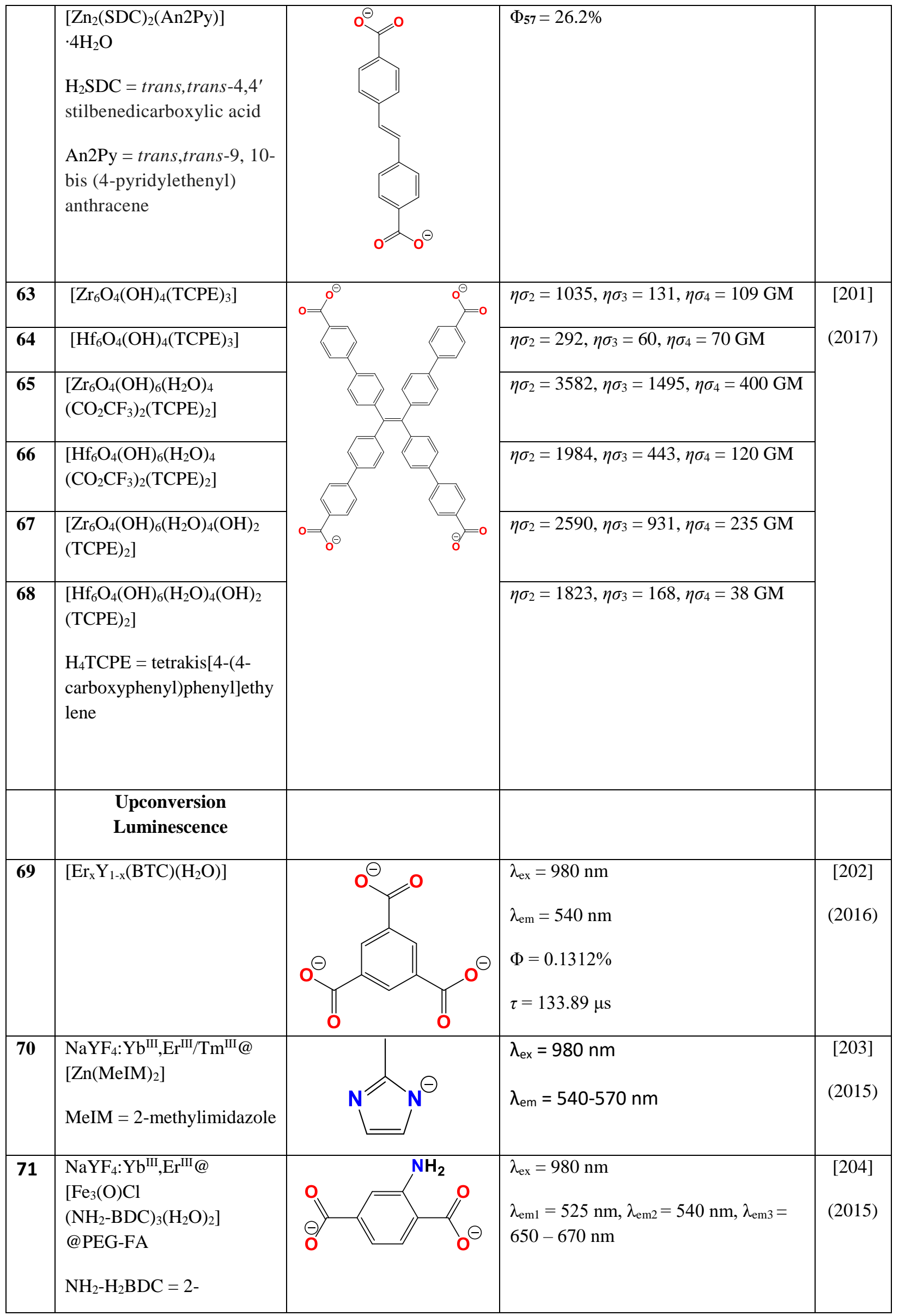




\begin{tabular}{|c|c|c|c|c|}
\hline & $\begin{array}{l}\text { aminoterephtahlic acid } \\
\text { PEG-FA = folic-acid- } \\
\text { modified polyethylene } \\
\text { glycol }\end{array}$ & & & \\
\hline 72 & $\begin{array}{l}\mathrm{NaYF}_{4}: \mathrm{Yb}^{3+}, \mathrm{Tm}^{3+} @ \\
{[\mathrm{Fe}(\mathrm{OH})(\mathrm{BDC})]} \\
\mathrm{H}_{2} \mathrm{BDC}=\text { terephthalic acid }\end{array}$ & ${ }_{0}^{0}$ & $\begin{array}{l}\lambda_{\mathrm{ex}}=980 \mathrm{~nm} \\
\lambda_{\mathrm{em} 1}=347 \mathrm{~nm}, \lambda_{\mathrm{em} 2}=352 \mathrm{~nm}, \lambda_{\mathrm{em} 3}= \\
452 \mathrm{~nm}, \lambda_{\mathrm{em} 3}=476 \mathrm{~nm}\end{array}$ & $\begin{array}{l}\text { [205] } \\
(2017)\end{array}$ \\
\hline 73 & $\begin{array}{l}{[\mathrm{Zn}-\mathrm{ADB}] /[\mathrm{Zn}(\mathrm{Pd}-\mathrm{DCP})]} \\
\mathrm{H}_{2} \mathrm{ADB}=4,4^{\prime}- \\
\text { (anthracene- } 9,10 \text {-diyl) } \\
\text { dibenzoic acid } \\
\\
\mathrm{H}_{2} \mathrm{Pd}-\mathrm{DCP}=\text { palladium } \\
5,15 \text {-diphenyl-10,20-di(4- } \\
\text { carboxyphenyl) porphyrin }\end{array}$ & & $\begin{array}{l}\lambda_{\mathrm{ex}}=532 \mathrm{~nm} \\
\lambda_{\mathrm{em} 1}=425-500 \mathrm{~nm} \\
\Phi=1.8 \% \\
\tau_{1}=430 \mu \mathrm{s}, \tau_{2}=180 \mu \mathrm{s}\end{array}$ & $\begin{array}{l}\text { [206] } \\
\text { (2016) }\end{array}$ \\
\hline & $\begin{array}{l}\text { : nonlinear optical coe } \\
\text { (the product of the ab } \\
\text {-Mayer, } 1 \mathrm{GM}=10^{-50}\end{array}$ & action $\mathrm{c}$ & $\begin{array}{l}\text {-section of two-, three-, and four-ph } \\
\text { luminescence quantum yield }(\eta)) ; C\end{array}$ & \\
\hline
\end{tabular}

\subsection{Photophysics of Nonlinear Optical Effects}

NLO effects arise due to nonlinear interactions between light and matter, which typically manifest when materials are exposed to high-intensity laser irradiation. NLO materials have been investigated intensively since Franken and coworkers' discovery of second-harmonic generation in 1961, and there is a wealth of literature detailing the various NLO processes, as well material design strategies that can be applied to exploit them. In this section, we touch upon the elementary concepts of NLOs at an introductory level, for the purpose of highlighting the differences between NLO and UCL. We thus introduce the earliest-observed and most commonly exploited NLO phenomena, namely second-harmonic generation (SHG), and two-photon absorption (TPA), and discuss the pioneering examples of these phenomena in MOFs, before shifting the focus to UCL mechanisms with recent examples from MOF 
literature. For a more detailed description of the various complex NLO phenomena and further examples of NLO-active MOFs, the reader is directed to the seminal text on NLO by R. Boyd [207], and several comprehensive reviews of NLO in MOFs and coordination polymers $[24,39,208]$.

\subsubsection{Second Harmonic Generation}

The interaction of light with a material generates a time-varying polarization response, i.e. a dipole moment $P$, in the material, the strength of which increases proportional to the opticalfrequency electric field $E$ of the incident light at low irradiance values. The constant of proportionality in this relationship, described by equation 6 , is a material parameter called the linear susceptibility, $\chi$. Equation 6 can be generalized by applying a power series expansion (equation 7), which contains terms with higher order, nonlinear dependences on $E$ that tend to dominate the response when a material is pumped using high laser intensities, which in the case of MOFs can range in order of magnitude from $10^{5}$ to $10^{10} \mathrm{Wcm}^{-2}$. From the $2 \omega$ factor that arises in the second-order term of the expansion, where $\omega$ is the frequency of the incident or pump beam, it is evident that a beam with two times the frequency, i.e. the secondharmonic of the pump, is generated in the material when it is exposed to such conditions; this is the basis of SHG.

$\boldsymbol{P}=\varepsilon_{0} \chi \boldsymbol{E}$, where $\boldsymbol{E}=E e^{i \omega t}+E^{*} e^{-i \omega t}$

$\boldsymbol{P}=P_{0}+\varepsilon_{0} \chi^{(1)} \boldsymbol{E}+\varepsilon_{0} \chi^{(2)}\left[E^{2} e^{2 i \omega t}+2|E|^{2}+E^{* 2} e^{-2 i \omega t}\right]+\cdots$

It is worth noting that the intensity of the second harmonic beam depends not only on the nature of the material, defined by its effective nonlinear optical coefficient $\left(d_{\mathrm{eff}}\right)$, but also on the fulfillment of phase-matching conditions (i.e. when the induced polarization and the 
generated electric field are in phase). These conditions can more easily be met in an NLO crystal of appropriate length as shown in equation 8 [209],

$I_{2 \omega} \sim \frac{2 \omega^{2} d_{e f f}^{2} I_{\omega}^{2}}{n_{2 \omega} n_{\omega}^{2} \lambda^{2}(\Delta k)^{2}}\left(\sin \frac{\Delta k l}{2}\right)^{2}$

where $I_{\omega}$ and $I_{2 \omega}$ are the intensities of the fundamental and second harmonic waves, $d_{\mathrm{eff}}$ is the effective nonlinear optical coefficient, $n_{\omega}$ and $n_{2 \omega}$ are the refractive indices at the fundamental and second harmonic, $l$ is the crystal thickness, $\lambda$ is the fundamental wavelength, and $\Delta k=k_{2 \omega}-2 k_{\omega}$ is the wave vector mismatch. The SHG intensity is maximized for the phase matched condition $\Delta k=0$.

SHG can be observed in non-centrosymmetric crystal materials having high polarizability and non-zero values of the non-linear susceptibility $\chi^{(2)}$, such as lithium niobate $\left(\mathrm{LiNbO}_{3}\right)$, potassium dihydrogenphosphate $(\mathrm{KDP})$, potassium titanyl phosphate $\left(\mathrm{KTiOPO}_{4}\right)$ and others, which are commonly exploited to generate new, shorter-wavelength coherent light for applications such as green lasers, tunable lasers, femtosecond pulsed lasers, and generating visible light from infrared light.

Since the 1990s, noncentrosymmetric MOFs have been designed and investigated for their SHG efficiency, and several examples have been reported with comparable or higher nonlinear optical coefficients than some popular commercial NLO materials [210]. Common strategies include designing chiral or acentric diamondoid nets, which owing to their noncentric tetrahedral nodes, tend to form non-centrosymmetric structures. SHG active MOFs crystallized in chiral space groups can be synthesized using either achiral components, or enantiopure or racemic mixtures of chiral ligands [211-213]. Acentric diamondoid structures on the other hand, can be synthesized by coordinating asymmetrical ligands to tetrahedrally connected metal centers such as $\mathrm{Zn}^{\mathrm{II}}, \mathrm{Cd}^{\mathrm{II}}$, or other $\mathrm{d}^{10}$ metals, which have forbidden $\mathrm{d}-\mathrm{d}$ 
transitions so as to avoid optical losses from visible light absorption in the material. This strategy was pioneered by Wenbin Lin's group, who reported a technique to crystal engineer the formation of non-centrosymmetric diamondoid structures by controlling the length of rigid $p$-pyridinecarboxylate ligands to ensure an odd number of interpenetrating nets [194]. The hydro(solvo)thermal treatment of $\mathrm{Zn}\left(\mathrm{ClO}_{4}\right) \cdot 6 \mathrm{H}_{2} \mathrm{O}$ and 4-cyanopyridine (which is in situ hydrolyzed into 4-pyridinecarboxylic acid) resulted in single crystals of $\mathbf{5 1}$ with a nonlinear coefficient $d_{\text {eff }}$ of $\sim 3$ times that of KDP - one of the most widely used and technologically significant non-linear crystal materials for photonics applications (Figure 26). Similar treatment of $\mathrm{Cd}\left(\mathrm{ClO}_{4}\right) \cdot 6 \mathrm{H}_{2} \mathrm{O}$ with trans-4-pyridylacrylic acid resulted in 52, which has a similar SHG efficiency to KDP. An alternative approach reported by Liang et al. uses the ligand tetrakis[4-(carboxyphenyl)oxamethyl]methane, as the 4-connected node with a $\mathrm{Cd}^{\mathrm{II}}$ center, obtaining 2-fold (53) and 7-fold (54) interpenetrated, noncentrosymmetric, diamondoid MOFs with SHG responses of $\sim 0.6$ and $~ 1.1$ times of urea [195]. In 2005, Wang et al. reported a mixed-ligand design strategy for obtaining acentric MOF structures with controllable polarity and chirality. The basis of this strategy is to apply in-situ ligand generation, as this favors acentric crystallization [214]. The MOFs 55 and 56 were synthesized by solvothermally reacting 2-(2-pyridyl)-1,3,4-oxadiazole, which is in situ hydrolyzed into 2-pyridine carboxylic acid and 4-pyridine carboxylic acid, with zinc and cadmium acetates respectively, resulting in the self-assembly of colorless crystalline MOFs bridged by two asymmetric ligands [196]. The SHG efficiencies of $\mathbf{5 5}$ and $\mathbf{5 6}$ are reported to be approximately 3.5 and 1.5 times higher than that of KDP respectively. As a general rule, it has been found that MOFs with longer ligands tend to generate higher degrees of interpenetration and are more likely to have higher SHG efficiencies. A large number of examples exist in the literature, and a comprehensive review of these structures and underlying strategies has been written by Wang et al.[208]. 

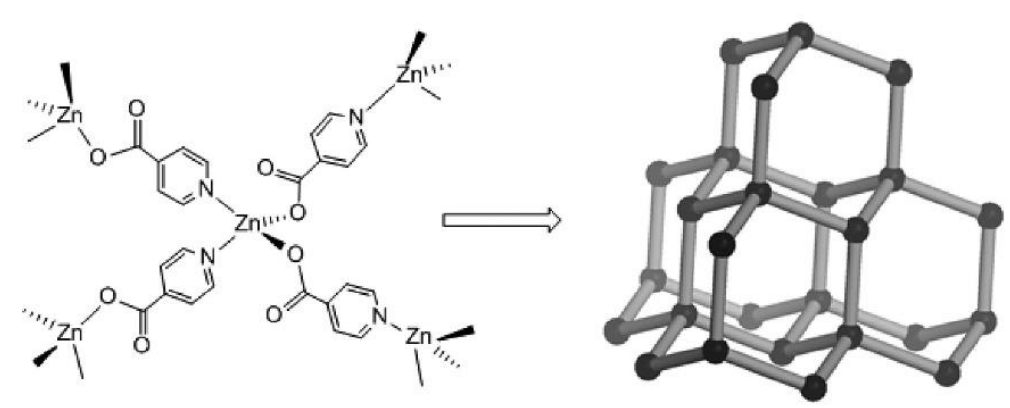

Figure 26. The diamondoid framework of 51. Reproduced with permission from reference [208].

In addition to crystal engineering strategies based on the selection of appropriate ligand and metal constituents, design of SHG active MOFs can also be approached from the molecular level, via the selection and capture of appropriate guest molecules. The $\chi^{(2)}$ parameter that is crucial for SHG is a function of the hyperpolarizability, $\beta$, of a molecule; a property that is enhanced in organic molecules that have an electron donor and acceptor separated by a conjugated bridge [215]. Such dipolar chromophores have the tendency to align in a centrosymmetric fashion due to dipole-dipole interactions, however, confining them within the pores of asymmetric porous MOFs or incorporating them into a directional coordination network are effective strategies to break this alignment, and have been shown to successfully induce SHG responses. The first example of this strategy was published by $\mathrm{Yu}$ et al., who encapsulated the ordered organic dipolar chromophore 4-(4-(diphenylamino)styryl)-1dodecylpyridinium bromide (DPASD) into the pores of 57 (also called ZJU-28) to obtain a high-intensity $532 \mathrm{~nm}$ beam upon exposure to a $1064 \mathrm{~nm}$ pump (Figure $27 \mathrm{a}-\mathrm{c}$ ) [197]. The SHG intensity of $\mathbf{5 7}$ is approximately 18.3 times higher than $\alpha$-quartz. Alternatively, donoracceptor substituted molecules with three-fold rotational symmetry ("octupolar" chromophores) are known to exhibit superior transparency versus optical nonlinearity than 
their dipolar counterparts, owing to additional components of molecular hyperpolarizability. The first example of such octupolar building blocks being assembled into an acentric bulk material was reported by Liu et al. in 2007. The solvothermal reaction of $\mathrm{CdCO}_{3}$ with $\mathrm{H}_{2} \mathrm{C}_{2} \mathrm{O}_{4}$ resulted in the formation of colorless crystals of the octupolar 3D complex $\left(\mathrm{H}_{2} \mathrm{NMe}_{2}\right)_{2}\left[\mathrm{Cd}_{3}\left(\mathrm{C}_{2} \mathrm{O}_{4}\right)_{4}\right] \cdot \mathrm{MeOH} \cdot 2 \mathrm{H}_{2} \mathrm{O}(\mathbf{5 8})$ (Figure $27 \mathrm{~d}-\mathrm{e}$ ) that exhibits SHG intensity approximately 15 times higher than KDP [198].

a)

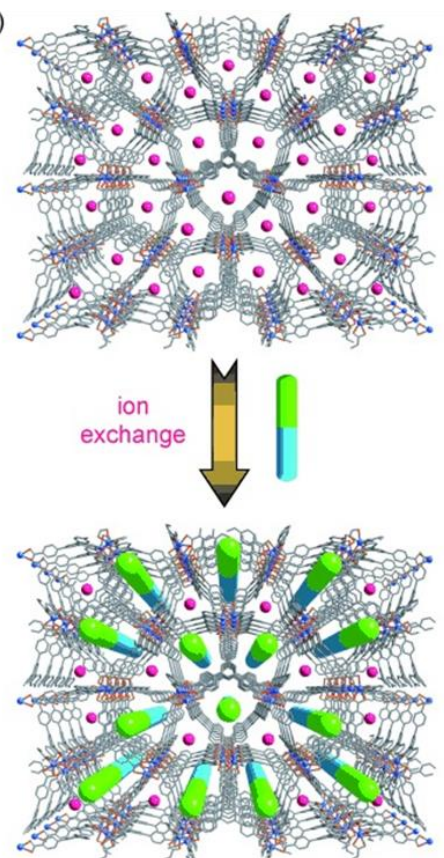

b)

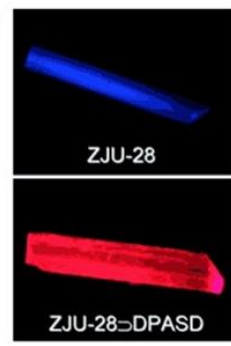

- $=\mathrm{Me}_{2} \mathrm{NH}_{2}^{+}$
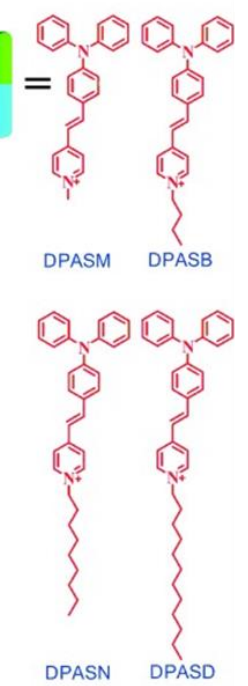

DPASN DPASD

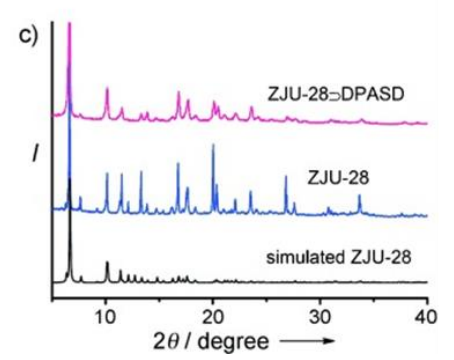

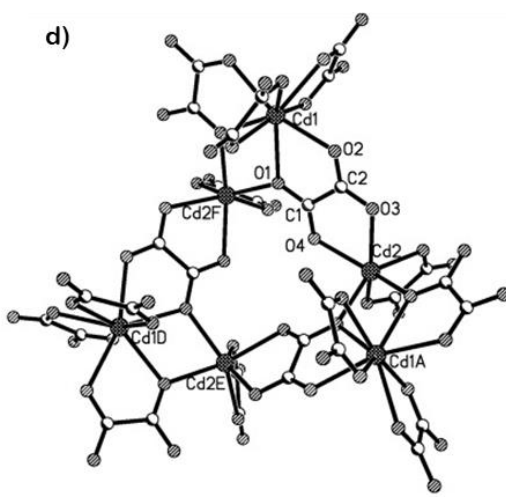

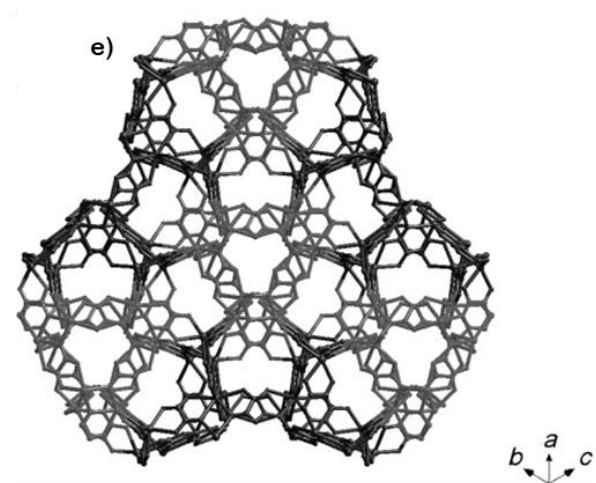

Figure 27. a) Schematic illustration of pyridinium hemicyanine chromophores incorporated into 57; b) Fluorescent microscope images of 57 and DPASD@57 illuminated with light of $365 \mathrm{~nm} \mathrm{UV}$; c) PXRD patterns of 57 and DPASD@ 57; d) The $\left[\mathrm{Cd}_{6}\left(\mathrm{C}_{2} \mathrm{O}_{4}\right)_{8}\right]^{4-}$ octupolar building block in 54; e) a view of the octupolar 3D anionic open framework of $\mathbf{5 4}$ Reproduced with permission from reference [197, 198]. 
It is important to note that similarly to SHG, the third-order term in the power series expansion of equation 6 gives rise to a third-harmonic generation (THG) response, whereby photons with a frequency of $3 \omega$ are emitted from a material upon application of a field with frequency $\omega$. Unlike with SHG, THG is not limited by symmetry and can be observed in both, centrosymmetric and non-centrosymmetric materials, however typical $\chi^{(3)}$ values are several orders of magnitude lower than $\chi^{(2)}$ values; this fact, coupled with the requirement to meet phase-matching conditions makes direct THG challenging. In 2016, Liu et al. reported efficient direct THG in a $\left[\mathrm{Zn}_{2}(\mathrm{SDC})_{2}(\mathrm{An} 2 \mathrm{Py})\right] \mathrm{MOF}(\mathbf{6 0})$ with a $\chi^{(3)}$ value 3 times that of $\alpha$ quartz, and exhibits photoluminescence of three times the incident frequency, with a cubic power dependence [216].

\subsubsection{Two-photon Absorption}

Another important NLO phenomenon is two-photon absorption (TPA), which occurs in both centrosymmetric and non-centrosymmetric materials, when the simultaneous absorption of two photons creates an energy transition equal to the sum of their energies. This phenomenon is often seen when a pulsed laser beam is tightly focused into a material so that incident photons overlap both spatially and temporally. Applications of TPA span from fluorescence imaging to optical data storage and few examples have been reported of these phenomena in MOFs. The rate of transitions that occur due to two-photon absorption from a single source is given by equation 9 ,

$R=\frac{\sigma_{2} I^{2}}{\hbar \omega}$

where $\sigma_{2}$ is the two-photon absorptivity and a material parameter that depicts the strength of the TPA process, $\hbar \omega$ is the energy of the incident photons, and $I$ is the intensity of the incident laser [217]. Since most known materials with TPA capabilities possess small values of $\sigma_{2}$, TPA, like SHG, only becomes significant at high optical intensities. 
Multi-photon absorption has been exploited for NLO anti-Stokes emissions in several MOFs. Quah et al. demonstrated a MOF based on simultaneous multi-photon excitation of a NLOactive ligand in 2015 [200]. The trans,trans-9,10-bis(4-pyridylethenyl) anthracene (An2Py) ligand that was used has a large absorption cross-section, a symmetric acceptor $-\pi-$ donor $-\pi-$ acceptor structure, which is typical of molecules with high second hyperpolarizability, and an open-shell singlet character that was characterized by electron paramagnetic resonance (EPR) spectroscopy. All three of these properties are known to yield enhancement in multiphotonexcited photoluminescence. Quah et al. synthesized an An2Py - $\mathrm{Zn}^{\mathrm{II}}$ MOFand investigated the impact of encapsulating high-quantum-yield guest molecules into their voids (60-62) (Figure 27). When irradiated with 150-femtosecond laser pulses at three different NIR wavelengths $(800 \mathrm{~nm}, 1200 \mathrm{~nm}$ and $1500 \mathrm{~nm})$, all three MOFs exhibit an absorption peak at $400 \mathrm{~nm}$, indicating two-photon, three-photon and four-photon absorption respectively. The fact that the multi-photon emission signal increases with excitation intensity further confirms multi-photon absorption as the luminescence mechanism in these MOFs. In the absence of the guest molecules, the MOF exhibits a threefold higher quantum yield (17.3\%) than that of the photo-active ligand alone $(6.2 \%)$, an enhancement that is attributed to the structural rigidity of the MOF minimizing unwanted quenching effects that stem from ligand aggregation. The fluorescence enhancement and quantum yield are further improved by the presence of the chosen guest molecules, perylene and anthracene, which emit in the excitation regions of the encapsulating MOF, leading to Förster resonance energy transfer (FRET) between the two. Anthracene emission has a higher overlap with the MOF than perylene emission, as evidenced by the considerably higher photoluminescence intensity in the anthracene-hosting MOF. More recently, in 2017, Medishetty et al. reported a series of MOFs (63-68) with record-high multi-photon absorption cross-sections, as well as a design guideline of five important criteria to achieve these properties in MOFs, including the 
selection of chromophore ligands with narrow one- and two-photon absorption bands, high fluorescence quantum yields, planar structural motifs and molecular dipole or multipolar structures to enhance polarization of the charge distribution. The combination of carefully selected ligands with zirconium and hafnium metal oxide clusters resulted in the formation of structures 63-68 with two-photon cross-section values of up to 3600 GM [201].
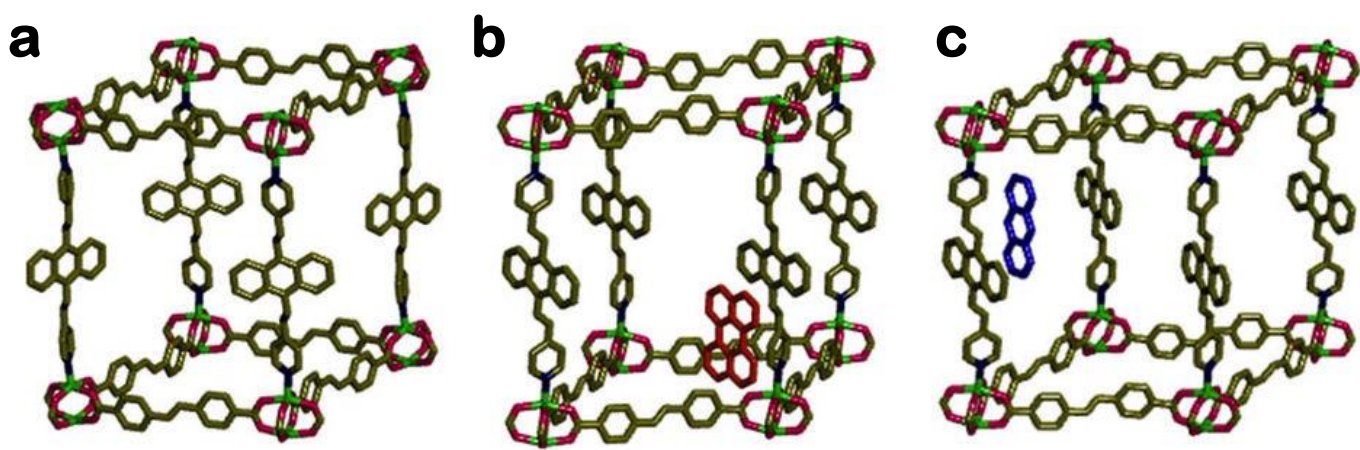

\begin{tabular}{|c|c|c|c|}
\hline d Compound & $800 \mathrm{~nm}$ & $1,200 \mathrm{~nm}$ & $1,500 \mathrm{~nm}$ \\
\hline An2Py & & & \\
\hline Compound 1a & & & \\
\hline Compound 2 & & & \\
\hline Compound 3 & & & \\
\hline $\mathrm{H}_{2}$ SDC & & & \\
\hline
\end{tabular}

Figure 27. a)-c) Views of 60-62 showing without interpenetration, with encapsulated guest molecules b) perylene (orange) and c) anthracene (blue); d) luminescence photos of 60-62 at 800,1200 , and $1500 \mathrm{~nm}$ femtosecond pulsed laser excitation. Reproduced with permission from reference [200]. 
It is worth noting that at high incident intensities, the energy absorbed by certain materials can also create structural changes that are spatially confined to the areas exposed to the laser focus. This phenomenon is commonly exploited for the laser direct writing of $3 \mathrm{D}$ structures in the bulk of materials to realize 3D micro- and nanostructures for optical data storage, among other applications. Exploiting this phenomenon, Yu et al. reported a novel two-photon responsivity in a multivariate MOF, 59 (also called ZJU-56-0.2), formed by introducing 20 mol\% of the zwitterionic 2,5-bis(3,5-dicarboxyphenyl)-1-methylpyridinium hydroxide ligand into the reaction solution of ZJU-56 [199]. The resulting high $\sigma_{2}$ crystals were patterned via 3D direct femtosecond laser writing to generate fluorescent, optically readable 3D patterns with micron feature resolution (Figure 28). 


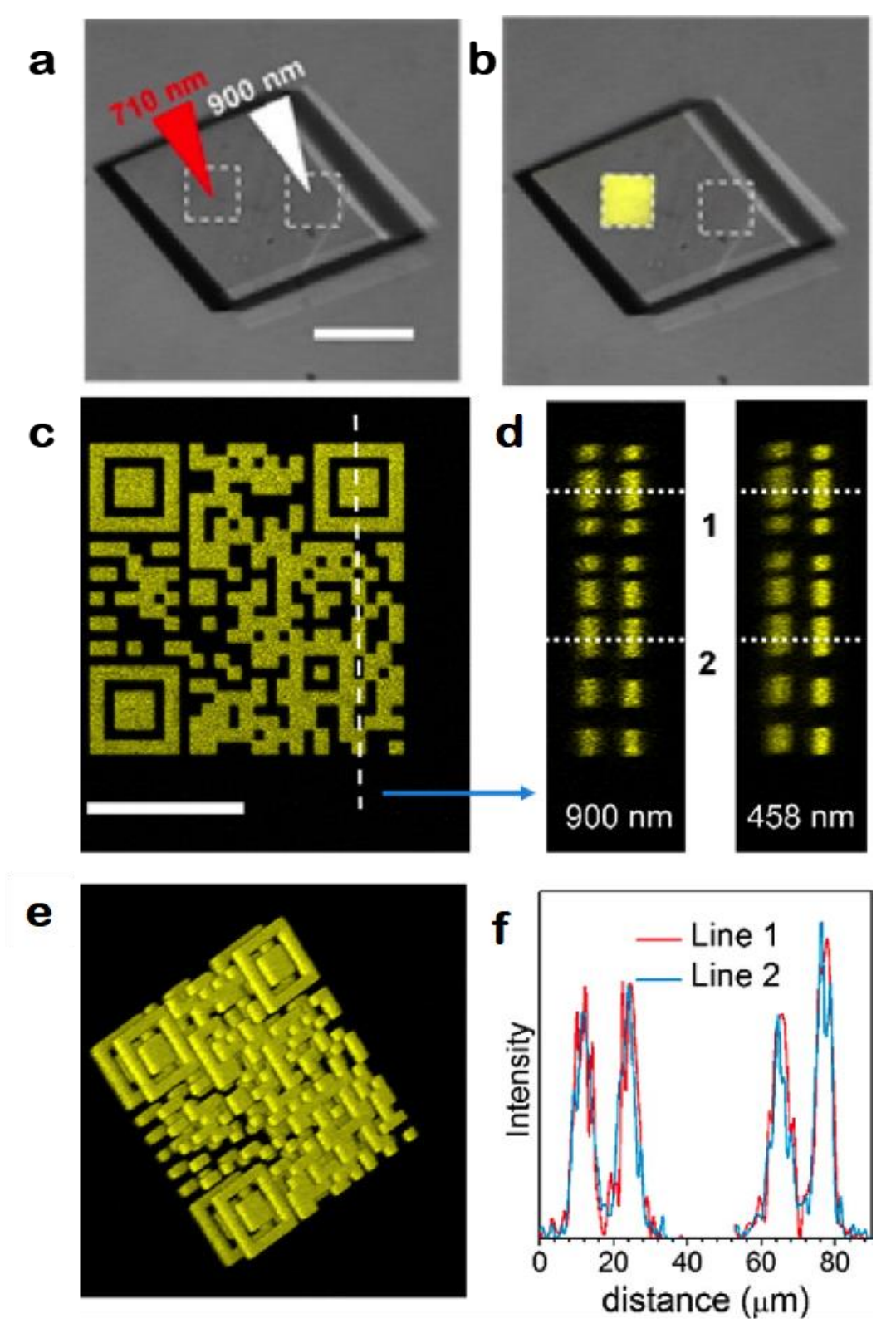

Figure 28. a) Bright-field image of a single crystal of $\mathbf{5 9}$ and two selected regions that were exposed to a tunable laser at wavelengths of 710 and $900 \mathrm{~nm}$, respectively; b) Bright-field and fluorescence emerged images of this crystal after laser exposure; c-d) Top and lateral views of two-photon excited fluorescent 2-D code stack; e) Three-dimensional reconstructed image of the stacked 2D code pattern; f) Intensity profiles of the fluorescent codes along lines 1 and 2 in the panels in d); Reproduced with permission from reference [199].

There are several design routes to realizing NLO effects including SHG, THG and TPA in MOFs, with key design strategies and examples of NLO MOFs reviewed in recent years [24, 
39, 208]. While promising examples do exist in the literature, there are challenges to achieving efficient anti-Stokes emission via NLO interactions in MOFs. For example, in the cases of SHG and THG, these can dominate the output response only if: i. a sufficiently highintensity pump is used, and ii. the wave-vectors of the induced polarization and the generated optical field are matched; otherwise these signals will drift in and out of phase, causing cancellations in the output signal [207]. These conditions necessitate the use of powerful laser sources and complex optical setups that can be used to ensure phase matching conditions are met. Overall, a great deal of further work is needed to realize NLO-active MOFs that have sufficient chemical, mechanical and thermal stability, optical transparency and (in the case of SHG and THG) phase match ability before they can be considered to replace the industrially available crystals.

\subsection{Upconversion Luminescence}

UCL processes, unlike the NLO phenomena described above, are a class of anti-Stokes phenomena that i) in many cases, can be achieved using inexpensive, low power, and sometimes even incoherent light sources, and ii) do not require phase-matching conditions to be met [218], making them promising for a broad range of applications including lasers, light sources, sensors, and biological imaging tools [219-221]. In particular, UCL has recently gained importance as a promising solution to the efficiency limits of sunlight-powered devices such as photovoltaic and photoelectrochemical cells, which absorb poorly at low frequencies [222-224]. The general principle of an upconversion process involves photoexcitation of a luminescent ion or molecule from its ground state to an intermediate excited state, followed by a second excitation via either photon absorption or energy transfer that promotes it to a higher-lying energy state. Radiative relaxation from this higher-energy state results in the emission of radiation with higher energy than that used for excitation. UCL takes place in certain ions, ion pairs and molecules with energy structures that include real 
intermediate states having long enough lifetimes $(\sim \mu \mathrm{s})$ to enable the accumulation of excited electrons. The sequential, rather than simultaneous, nature of the excitation, negates the need for excessively high excitation intensities, and can be achieved using powers that are 5-10 orders of magnitude lower that those required for SHG or TPA [225]. Broadly, there are two possible approaches to achieving low-power upconversion in MOFs. The first exploits lanthanide ions, in particular the energy transfer that takes place between $\mathrm{Yb}^{\mathrm{III}}$ and $\mathrm{Er}^{\mathrm{III}}$. The second is a triplet-triplet annihilation, which is a ligand-based approach to upconversion MOF design, and exploits a cascade of energy transfer events that take place in carefully selected pairs of chromogenic molecules.

\subsubsection{Lanthanide-based upconversion}

Some of the most common examples of efficient UCL materials are lanthanide ( $\left.\mathrm{Ln}^{\mathrm{III}}\right)$ ions. $\mathrm{Ln}^{\mathrm{III}}$ ions possess unique photophysical properties such as fixed energy levels, sharp emission lines, long luminescence lifetimes, and high photostability. $\mathrm{Ln}^{\mathrm{III}}$-based UCL can be a singleion or a multi-ion process; the ion that emits the output photon is called the activator, whilst the ion that is first excited in the case of multi-ion process is conventionally referred to as the sensitizer. There are essentially three physical mechanisms of $\mathrm{Ln}^{\mathrm{III}}$-based UCL; excited state absorption (ESA), energy transfer upconversion (ETU), cooperative transfer upconversion (CTU) (Figure 29). These are described below.

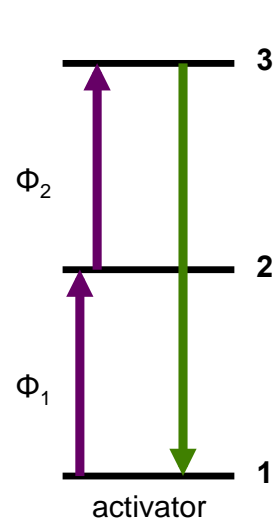

(a)

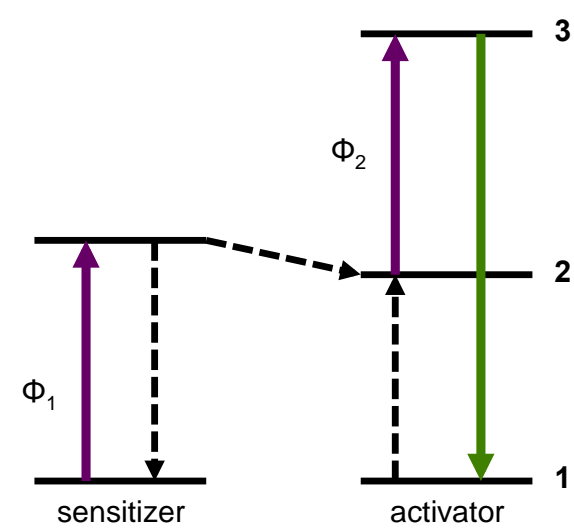

(b)

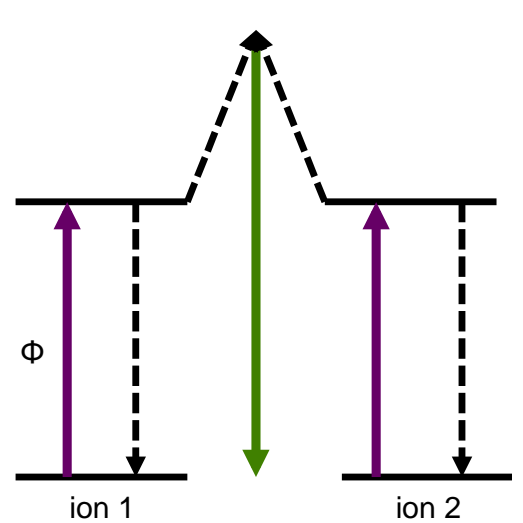

(c) 
Figure 29. Upconversion luminescence processes a) Excited state absorption (ESA); b) Energy transfer upconversion (ETU); c) Cooperative transfer upconversion (CTU). Solid lines represent photon absorption (purple) and emission (green). Dashed lines represent energy transfer.

ESA occurs when an ion or molecule that has been excited by a pump photon to a real, metastable intermediate excited state is then promoted to a higher excited state by the subsequent absorption of a second photon (Figure 28 a). Relaxation from the higher-lying state results in the emission of a higher energy photon than either of those used in the excitation process. ESA hence occurs in a single ground-state ion. Typical ions that exhibit high-efficiency ESA include $\mathrm{Nd}^{\mathrm{III}}$, $\mathrm{Er}^{\mathrm{III}}$, and $\mathrm{Tm}{ }^{\mathrm{III}}$. Materials used to exploit ESA mainly consist of a low concentration of lanthanide activators doped in a bulk solid or a coordination complex. The efficiency of ESA emission from such materials depends on the lifetime of the intermediate metastable states, as well as on the concentration of activator ions. A $<1 \%$ concentration of activator molecules in a bulk solid is often desirable to prevent crossrelaxation between activators, and to increase the gain in the excited-state absorption process. While ESA is a single-ion, sequential absorption process, ETU, first hypothesised by Auzel in 1966, involves what is essentially a Dexter energy transfer from a sensitizer to an activator that is already in an excited state (Figure $28 \mathrm{~b}$ ). As a Dexter transfer process, ETU requires a wavefunction overlap between the sensitizer and activator, and hence typically occurs over short distances $<10 \AA$, requiring a high enough concentration of sensitizers and activators with comparable energy levels. Cooperative transfer upconversion (CTU) processes either take the form of cooperative sensitization or cooperative luminescence. In cooperative luminescence, Coulomb coupling between a pair of ions facilitates a transition in which they simultaneously depopulate their excited states to emit a single photon [226]. These transitions 
occur in spectral regions where the individual ions do not have absorption or emission, and in cases where ETU mechanisms cannot occur, such as in low sensitizer/activator concentrations, as well as systems where the paired ions have too large an energy level mismatch. In contrast to cooperative luminescence, cooperative sensitization is when a single photon simultaneously excites two ions. This process was first elucidated in a series of experiments performed by Varsanyi and Dieke in 1961 [227], where $\mathrm{LnCl}_{3}$ crystals, in which the $\mathrm{Ln}^{\mathrm{III}}$ is replaced by a range of lanthanide ions, were exposed to monochromatic light of varying excitation wavelengths, and the resulting fluorescence spectra were recorded. It was observed that the fluorescence spectrum of these crystals is independent of excitation wavelength, and remains constant even when excited at high energies where a single ion is not capable of absorption. Further analysis revealed that fluorescence is seen only when the crystal is excited at wavelengths such that the difference between the energy of the exciting photon and that of the stable fluorescent level in one ion is comparable to the energy of a lower excitation level in a neighboring ion, revealing the mechanism in which a single photon excites two ions simultaneously. Fluorescence was observed at excitation frequencies required to simultaneously excite one $\operatorname{Pr}{ }^{I I I}$ ion to the ${ }^{3} \mathrm{P}_{0}$ state and a second ion to one of the lower states. The year following this experimental demonstration, Dexter published a theoretical description of the process that proves a non-zero probability of cooperative luminescence occurring between ions, even in the absence of overlapping wavefunctions [228].

While the rational design and synthesis of a material that exhibits a specific one of these processes over another is challenging, a thorough understanding of the various possible mechanisms is necessary in order tounderstand and better exploit the upconversion processes that occur. Lanthanide-based UCL has been observed in both single-center materials and cooperative systems that consist of a pair of lanthanides, with $\mathrm{Yb}^{\mathrm{III}}$ often acting as a sensitizer 
due to its large absorption cross-section at the fixed energy gap of $\sim 980 \mathrm{~nm}$, which allows it to effectively absorb near-infrared (NIR) photons and transfer the excitation energy to nearby activators. $\mathrm{Ho}^{\mathrm{III}}$ and especially $\mathrm{Er}^{\mathrm{III}}$ and $\mathrm{Tm}^{\mathrm{III}}$ are ideal ETU activators in which phononassisted or resonant ETU occurs easily, with the $\mathrm{Yb}^{\mathrm{III}} / \mathrm{Er}^{\mathrm{III}}$ system often exhibiting the highest efficiency. $\mathrm{Ln}^{\mathrm{III}}$ ions without metastable levels as energy storage reservoirs, such as $\mathrm{Tb}^{\mathrm{III}}$ and $\mathrm{Eu}^{\mathrm{III}}$, typically exhibit $\mathrm{CTU}$, with the excitation energy of two adjacent $\mathrm{Yb}^{\mathrm{III}}$ being simultaneously transferred to $\mathrm{Tb}^{\mathrm{III}}$ or $\mathrm{Eu}^{\mathrm{III}}$.

The majority of optically active MOFs reported in literature, including lanthanide-doped luminescent MOFs, respond to UV and visible, but not NIR wavelength light. The design and synthesis of NIR-excitable MOFs therefore remains a challenge, but one with many promising applications ranging from energy conversion and storage to bioimaging and sensing. Recent years have seen few key successes in the synthesis and characterization of upconversion MOFs and MOF-based upconversion systems. Zhang et al. recently demonstrated characteristic emissions of 520, 540 and $651 \mathrm{~nm}$ in $\mathrm{Er}^{\mathrm{III}}$-doped lanthanide "UpMOFs" (69), under $980 \mathrm{~nm}$ excitation [202]. The UCL intensities of these $\left[\operatorname{Ln}(\mathrm{BTC})\left(\mathrm{H}_{2} \mathrm{O}\right)\right] \cdot \mathrm{DMF}$ frameworks $\quad$ where $\mathrm{Ln}=\mathrm{Er}^{\mathrm{III}}, \quad \mathrm{Y}^{\mathrm{III}}, \quad \mathrm{BTC}^{3-}=1,3,5-$ benzenetricarboxylate) vary with an $\mathrm{Er}^{\mathrm{III}}$ mole fraction in Ln ranging from $2 \%-100 \%$, as shown in Figure 30. Here, $\mathrm{Y}^{\mathrm{III}}$, with its lack of ${ }^{4} \mathrm{f}$ orbitals, does not contribute to the UCL output and acts only as an ion diluent to facilitate the investigation of the relationship between $\mathrm{Er}^{\mathrm{III}}$ concentration and UCL emission intensity. The characteristic emissions from ErIII are attributed to the electronic transitions from ${ }^{2} \mathrm{H}_{11 / 2} \rightarrow{ }^{4} \mathrm{I}_{15 / 2}(520 \mathrm{~nm}),{ }^{4} \mathrm{~S}_{3 / 2} \rightarrow$ ${ }^{4} \mathrm{I}_{15 / 2}(540 \mathrm{~nm})$ and ${ }^{4} \mathrm{~F}_{9 / 2} \rightarrow{ }^{4} \mathrm{I}_{15 / 2}(651 \mathrm{~nm})$, with $6 \%$ being the optimal concentration of $\mathrm{Er}{ }^{\mathrm{III}}$, beyond which the effect of concentration quenching dominates over UCL. The energy transfer process occurs due to the absorption of a $980 \mathrm{~nm}$ pump photon, which excites the $\mathrm{Er}^{\mathrm{III}}$ to the ${ }^{4} \mathrm{I}_{11 / 2}$ energy level, at which point a second $980 \mathrm{~nm}$ photon is absorbed, promoting 
it to the ${ }^{2} \mathrm{H}_{11 / 2}$ level. Subsequent non-radiative relaxation demotes the ion to the lower-lying ${ }^{4} \mathrm{~S}_{3 / 2}$ energy level, thus leading to the radiative $520 \mathrm{~nm}$ and $540 \mathrm{~nm}$ green emissions. The weaker $651 \mathrm{~nm}$ emission that occurs is explained by relaxation through the route of ${ }^{2} \mathrm{H}_{11 / 2} \rightarrow$ ${ }^{4} \mathrm{~S}_{3 / 2} \rightarrow{ }^{4} \mathrm{~F}_{9 / 2} \rightarrow{ }^{4} \mathrm{I}_{15 / 2}$. ESA rather than ETU was confirmed as the upconversion mechanism in Up-MOFs by investigating a $\mathrm{Yb}^{\mathrm{III}}-\mathrm{Er}^{\mathrm{III}}$ co-doped system with $\mathrm{Y}^{\mathrm{III}}$, $\mathrm{Yb}^{\mathrm{III}}$, and $\mathrm{Er}^{\mathrm{III}}$ mole ratios of $60 / 30 / 10,30 / 60 / 10$ and 0/90/10. The same emission intensity was recorded for all variations of $\mathrm{Yb}^{\mathrm{III}}$ concentration, negating the possibility of ETU as the underlying UCL mechanism in these MOFs. This was further confirmed by the fact that increasing the power of the laser excitation source results in an increase in UCL emission given by $I_{f} \propto P^{n}$, where $I_{f}$ is the fluorescent intensity, $P$ is the excitation power and $n$ is the number of photons absorbed. The values of $n$ thus calculated in $\mathrm{Y}^{\mathrm{III}} / \mathrm{Er}^{\mathrm{III}} \mathrm{Up}-\mathrm{MOF}$ confirm that the ${ }^{4} \mathrm{~S}_{3 / 2}$ and ${ }^{4} \mathrm{~F}_{9 / 2}$ states were in fact populated via multi-photon processes. Even the most efficient of the Up-MOFs suffers from a low quantum yield of just $0.1312 \%$, which is a typical drawback of lanthanide-based upconversion systems, due to nonradiative relaxation processes that take place. 


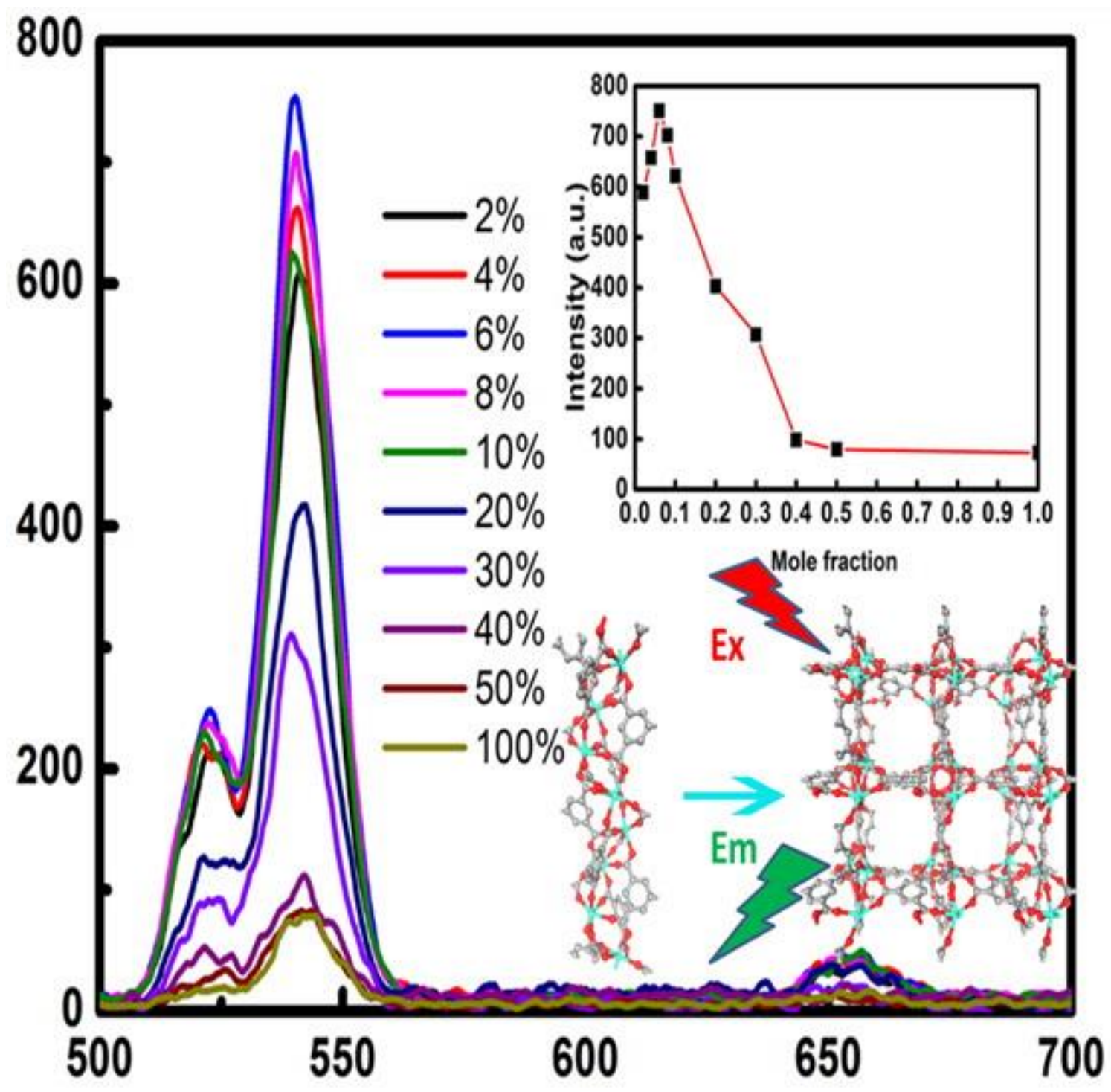

Figure 30. Upconversion luminescence spectra of 64 with different concentration of $\mathrm{Er}^{\mathrm{III}}$ (range of $\chi$ is from $2 \%$ to $100 \%$ ) with the excitation at $980 \mathrm{~nm}$; Inset: The optimal concentration of doped $\mathrm{Er}^{\mathrm{III}}$ ions is 6\%. Reproduced with permission from reference [202].

Prior to these first reports of MOFs that are inherently capable of UCL, the common strategy was to synthesize upconversion nanoparticle (UCNP)-MOF composites, several of which have been developed and investigated. The integration of nanoparticles into MOFs in general has generated interest due to its potential for enhancing MOF functionality, with applications ranging from catalysis and storage to biological and magnetic resonance imaging. In 2012, 
Lu et al. demonstrated a simple method of fabricating NP@MOF composites that circumvents the commonly faced problems of agglomeration, incomplete confinement or inhomogeneous distribution of NPs within a porous framework [229]. By carrying out the crystallization of ZIF-8 in a solvent containing surfactant-coated NPs, they succeeded in realizing a homogeneous composite with NPs (70) fully incorporated and well-dispersed within the framework, due to their successive adsorption onto the MOF surfaces as the crystals formed (Figure 31). The method was successfully demonstrated using NPs and hybrid crystals of various compositions including $\mathrm{Au}, \mathrm{Pt}, \mathrm{CdTe}, \mathrm{Fe}_{3} \mathrm{O}_{4}$ and lanthanide-doped $\mathrm{NaYF}_{4}$, the latter of which exhibited upconverted green emission when excited at $980 \mathrm{~nm}$.

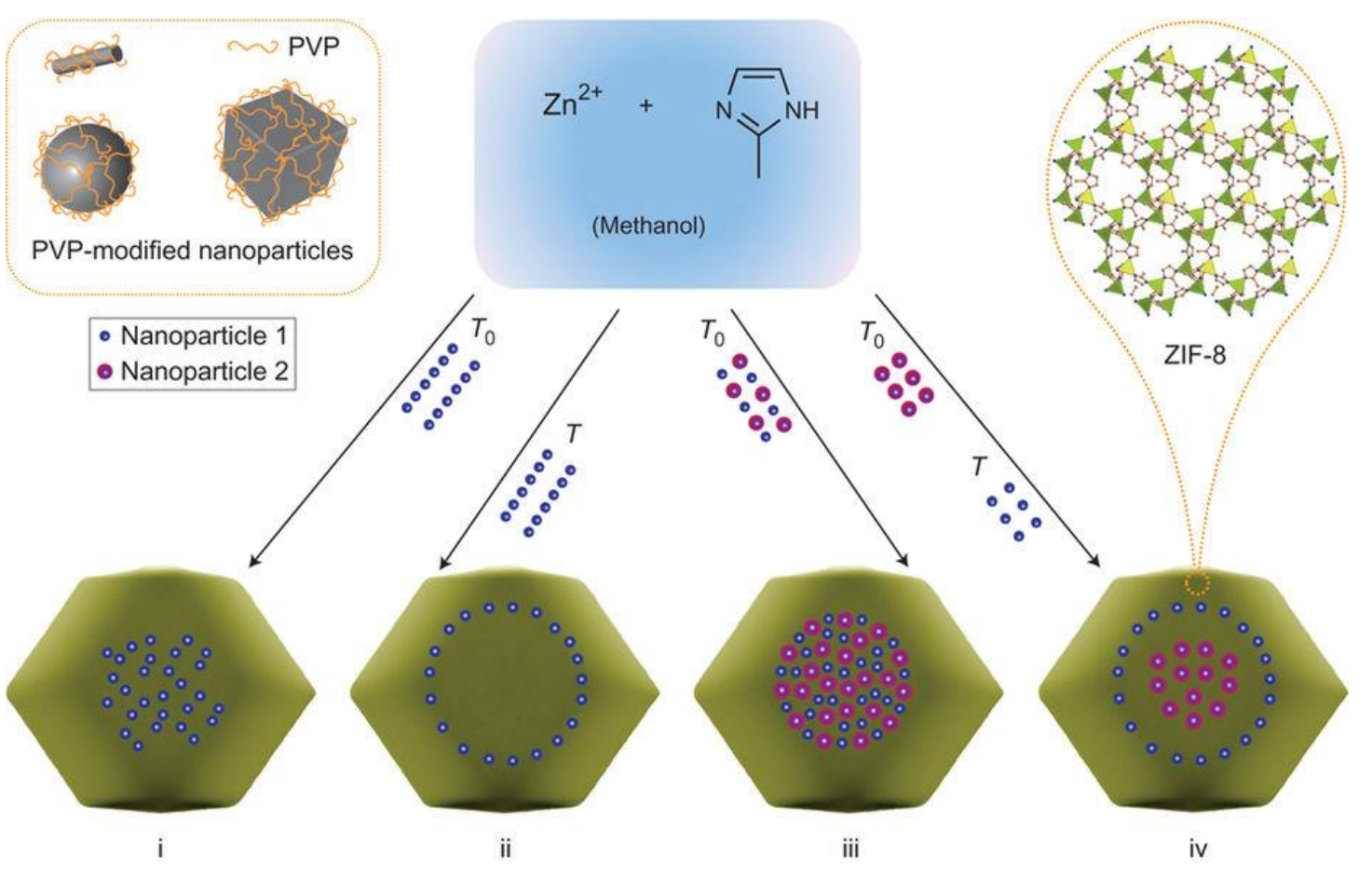

Figure 31. Scheme of the controlled encapsulation of nanoparticles in ZIF-8 crystals. Reproduced with permission from reference [229]. 
Several reports of lanthanide-doped $\mathrm{NaYF}_{4}$ NPs embedded in MOFs for UCL applications via similar synthesis steps were published in following years. $\mathrm{NaYF}_{4}$ is a well-known host for luminescent lanthanide ions that exhibits high-efficiency UCL owing to a low phonon cut-off frequency that suppresses nonradiative multiphonon relaxation. A detailed characterization of ZIF-8 embedded with $\mathrm{Yb}^{\mathrm{III}}(18 \%)$ and $\mathrm{Er}^{\mathrm{III}} / \mathrm{Tm}^{\mathrm{III}}-(2 \%)$ doped $\mathrm{NaYF}_{4} \quad \mathrm{NPs}$ $\left(\mathrm{NaYF}_{4}: \mathrm{Yb}^{\mathrm{III}}, \mathrm{Er}^{\mathrm{III}} / \mathrm{Tm} @ \mathrm{ZIF}-8\right)$ using the same method was reported by Liu and Yan [203] in 2015. When excited at 980nm, the emission spectrum of $\mathrm{NaYF}_{4}: \mathrm{Yb}^{\mathrm{III}}, \mathrm{Er}^{\mathrm{III}} @ \mathrm{ZIF}-8$ displays green bands between 510 - $540 \mathrm{~nm}$ associated with the ${ }^{2} \mathrm{H}_{11 / 2} \rightarrow{ }^{4} \mathrm{I}_{15 / 2}$ transition, and $540-$ $570 \mathrm{~nm}$ associated with the ${ }^{4} \mathrm{~S}_{3 / 2} \rightarrow{ }^{4} \mathrm{I}_{15 / 2}$ transition, as well as strong red emission between $640-690 \mathrm{~nm}$ arising from the ${ }^{4} \mathrm{~F}_{9 / 2} \rightarrow{ }^{4} \mathrm{I}_{15 / 2}$ transition. The resulting green-yellow luminescence from this composite nanostructure is seen in the inset of Figure 32 a. $\mathrm{NaYF}_{4}: \mathrm{Yb}^{\mathrm{III}}, \mathrm{Tm}^{\mathrm{III}} @ \mathrm{ZIF}-8$ on the other hand exhibits a violet luminescence (Figure $32 \mathrm{~b}$ ) that results from combined 440-490 $\mathrm{nm}, 640-680 \mathrm{~nm}$ and 750-850 nm emissions associated with the ${ }^{1} \mathrm{D}_{2} \rightarrow{ }^{3} \mathrm{~F}_{4}$ and ${ }^{1} \mathrm{G}_{4} \rightarrow{ }^{3} \mathrm{H}_{6}$, the ${ }^{1} \mathrm{G}_{4} \rightarrow{ }^{3} \mathrm{~F}_{4}$ and the ${ }^{3} \mathrm{H}_{4} \rightarrow{ }^{3} \mathrm{H}_{6}$ transitions respectively. When excited at 396 nm, NaYF $4: \mathrm{Yb}^{\mathrm{III}}, \mathrm{Er}{ }^{\mathrm{III}} @ \mathrm{ZIF}-8$ exhibits near-UV emission with a peak at 450 $\mathrm{nm}$ (Figure $32 \mathrm{c}$ ) that derives from the emission spectrum of the ZIF-8 host. The combined UCNP@MOF system is thus reported as a promising candidate for luminescence color tuning via selective excitation.
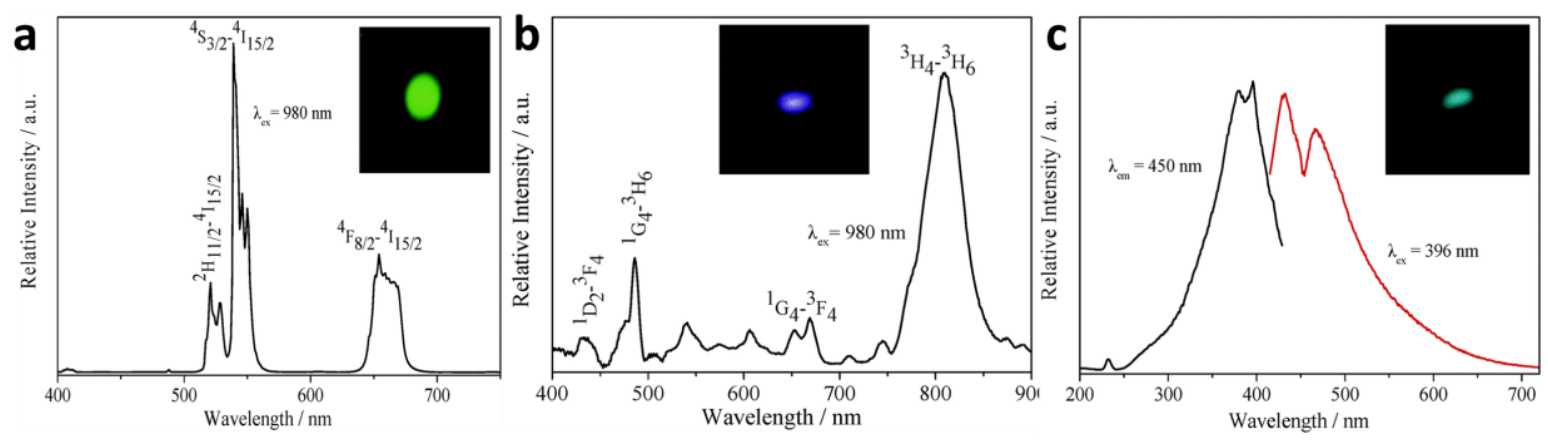
Figure 32: Room-temperature upconversion emission spectra and digital photographs of a) $\mathrm{NaYF}_{4}: \mathrm{Yb}^{\mathrm{III}} / \mathrm{Er}^{\mathrm{III}}$ and b) $\mathrm{NaYF}_{4}: \mathrm{Yb}^{\mathrm{III}} / \mathrm{Tm}^{\mathrm{III}}$ @ ZIF-8 nanocomposites; c) Luminescence excitation and emission spectra of $\mathrm{NaYF}_{4}: \mathrm{Yb}^{\mathrm{III}} / \mathrm{Er}^{\mathrm{III}}$ nanocomposite. Reproduced with permission from reference [203].

Li et al. integrated $\mathrm{NaYF}_{4}: \mathrm{Yb}^{\mathrm{III}}, \mathrm{Er}^{\mathrm{III}}$ UCNPs into a Fe-MIL-101-NH 2 nano-MOF (NMOF) (71) shell to create a core-shell nanocomposite with strong potential for use in combined UCL-magnetic resonance imaging (MRI) [204]. The MOF for this application was selected based on its $\mathrm{T}_{2}$-MRI properties. In MRI, proton concentration as well as tissue relaxation rates, defined by the $T_{1}$ and $T_{2}$ time constants, contribute to image contrast. $T_{1}$, or spinlattice relaxation, is related to the process by which longitudinal magnetization of a sample is recovered after an excitation pulse, and $\mathrm{T}_{2}$, or spin-spin relaxation, describes the time in which the decay of transverse magnetization occurs in a sample. It is possible to enhance the contrast in an MRI scan by inserting compounds that preferentially enter one tissue type over another and are then capable of altering the $\mathrm{T}_{1} / \mathrm{T}_{2}$ relaxation times of water protons in the tissue. The $\mathrm{NaYF}_{4}: \mathrm{Yb}^{\mathrm{III}}, \mathrm{Er}^{\mathrm{III}} @ \mathrm{Fe}-\mathrm{MIL}-101-\mathrm{NH}_{2}$ nanostructures were prepared by functionalizing first with polyethylene glycol (PEG) to enhance their stability and biocompatibility, and then with folic acid (FA) to promote targeted uptake by cancer cells. A dispersion of $\mathrm{NaYF}_{4}: \mathrm{Yb}^{\mathrm{III}}$,Er ${ }^{\mathrm{III}} @ \mathrm{Fe}-\mathrm{MIL}-101-\mathrm{NH}_{2} @ \mathrm{PEG}-\mathrm{FA}$ nanostructures (UMP-FAs) in water considerably reduces the $T_{2}$ relaxation time of water protons, from 2047 to $5.6 \mathrm{~ms}$, and $\mathrm{T}_{2}$-weighted images become increasingly darker with increasing UMP concentration.

Another dual application of upconverting $\mathrm{NaYF}_{4}: \mathrm{Yb}^{\mathrm{III}}, \mathrm{Er}^{\mathrm{III}} @ \mathrm{MOFs}$ was demonstrated by Deng et al. [230], who used the high porosity, non-toxic Fe-MIL-100 MOF functionalized with aptamer AS1411 as a shell for UCNPs in a composite designed for targeted drug delivery combined with diagnostic cell imaging. The exceptionally porous Fe-MIL-100 shells 
exhibited a high loading ratio (17.2 wt\%) of a model drug, doxycycline monohydrate (DOX), and a $\mathrm{pH}$-dependent release time of 4 days in neutral $(\mathrm{pH}=7.4) \mathrm{PBS}$ and $2 \mathrm{~h}$ in acidic $(\mathrm{pH}=$ 5.0) PBS. The AS114 functionalization was shown to promote selective uptake of the nanostructures by cancer cells, thus the complete system is described as a prototype diagnostic upconversion imaging and targeted drug delivery tool.

More recently, an UCNP-MOF core-shell structure was demonstrated for photocatalysis where the role of the UCNPs was to facilitate the exploitation of NIR light by MOF photocatalysts [205]. The core-shell structure consists of $\mathrm{NaYF}_{4}: \mathrm{Yb}^{\mathrm{III}}, \mathrm{Tm}^{\mathrm{III}} \mathrm{UCNPs}$ coated by Fe-MIL-53 that is grown layer-by-layer in solution with controllable thickness (72). The two components are complementary in the sense that the UCNPs absorb NIR radiation and then emit UV-Vis radiation, which in turn activates the MOF photocatalyst. Upon $980 \mathrm{~nm}$ excitation, the UCNPs alone emit UV and blue radiation at $347 \mathrm{~nm}, 362 \mathrm{~nm}, 452 \mathrm{~nm}$ and 476 $\mathrm{nm}$, all of which were significantly quenched after the NPs were coated in Fe-MIL-53, indicating absorption by the MOF (Fig. 33). Modifying the system with $\mathrm{NH}_{2}$ groups further enhances the absorption and subsequent photocatalytic activity due to the antenna effect pushing the absorption edge to $700 \mathrm{~nm}$. The mechanism of the core-shell system was studied by suspending it in a solution containing dye and analyzing the dynamics of dye degradation. NIR radiation is absorbed by the UCNPs and this excites electrons in the $\mathrm{Tm}^{\mathrm{III}}$ to higher energy levels. This energy is then transferred to the MOF shell via FRET, and the Fe ${ }^{\mathrm{III}}-\mathrm{O}$ clusters in the MOF produce photogenerated electrons $\left(\mathrm{e}^{-}\right)$and holes $\left(\mathrm{h}^{+}\right)$as a result. The photogenerated electrons migrate to the surface of the particles and interact with the surrounding $\mathrm{O}_{2}$ to create radicals that oxidize the dye, while the holes migrate to the surface of the particles and oxidize the dye directly. This process in addition to the UV and visible light activation of MIL-53(Fe) leads to an enhancement in the photocatalytic activity of the MOF (Figure 33). 

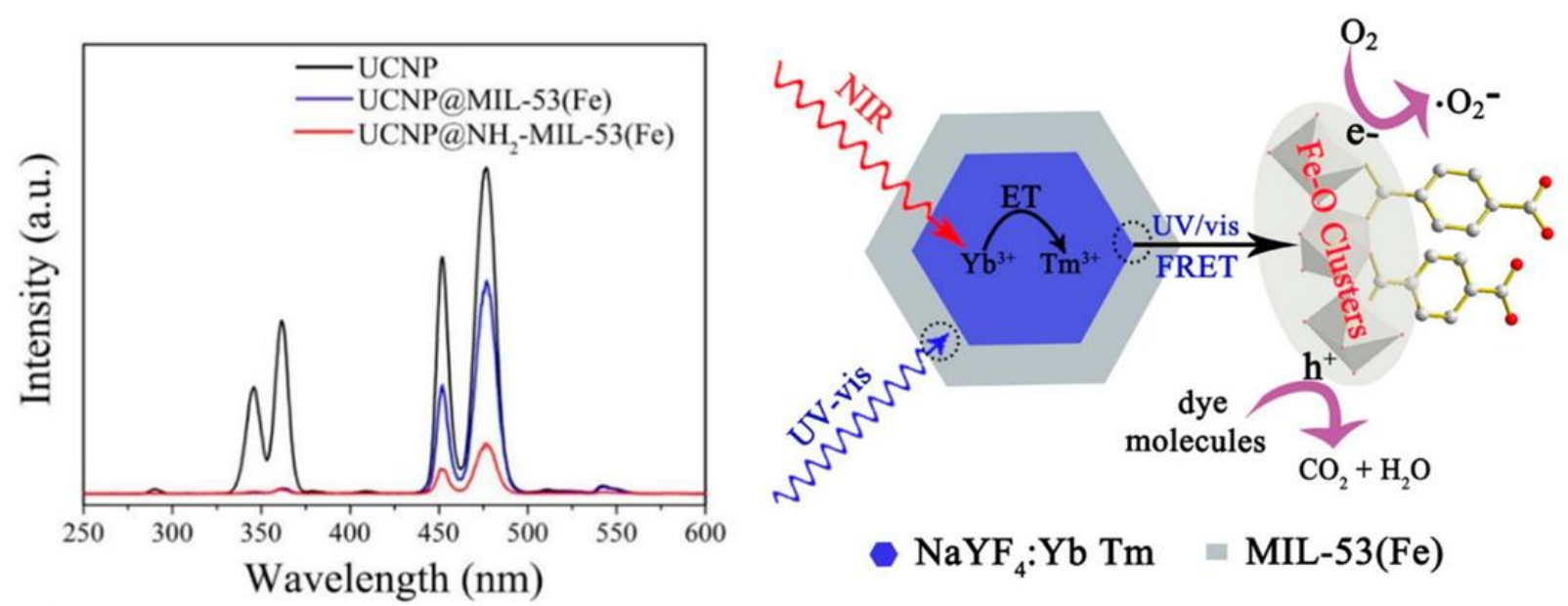

- $\mathrm{NaYF}_{4}: \mathrm{Yb} \mathrm{Tm}^{-\mathrm{MIL}-53(\mathrm{Fe})}$

Figure 33. a) UCL spectra of the prepared UCNPs, (72) UCNP@MIL-53(Fe), and UCNP@ $\mathrm{NH}_{2}-\mathrm{MIL}-53(\mathrm{Fe})$ under NIR excitation; b) mechanism for enhanced photocatalysis in UCNP-MOF system. Reproduced with permission from reference [205].

\subsubsection{Triplet-triplet annihilation upconversion}

Triplet-triplet annihilation (TTA) is a process that occurs between two types of organic molecules (one sensitizer and one activator), which are either dissolved together in a solution, or form part of a solid matrix. The sensitizer molecule absorbs low energy photons to generate triplet excited states, which are then transferred to the activators via triplet-triplet energy transfer (TET). When two activators interact via triplet-triplet annihilation, they effectively combine the two triplet excitons to create a higher-energy singlet state. This singlet state radiates a high-energy photon as it relaxes down to ground state (Figure 34). 


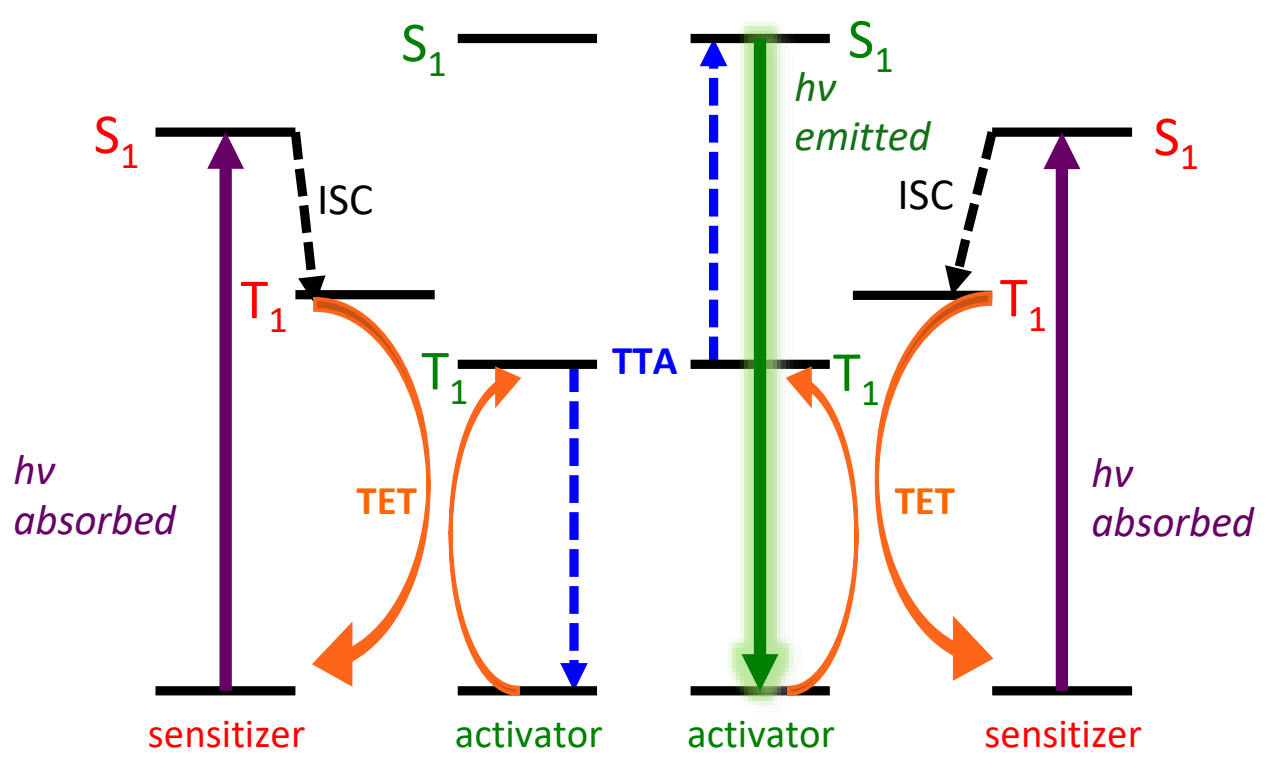

Figure 34. Mechanism for triplet-triplet annihilation upconversion process. $S_{1}$ represents the lowest singlet state, and $\mathrm{T}_{1}$ the lowest triplet state, of the sensitizer (red) and activator (green). A low-energy photon is absorbed by two sensitizer molecules (purple solid lines), which rapidly undergo intersystem crossing to occupy their lowest triplet states (black dashed lines). This energy is then transferred via triplet energy transfer to the lowest triplet of the activator molecules (orange curved lines), which undergo triplet-triplet annihilation (blue dashed lines) to emit a higher-energy photon than those used for excitation (green solid line).

Most commonly, high-efficiency TTA upconversion is observed in low-viscosity solvents, since intermolecular collisions encourage energy transfer processes in both, sensitizerannihilator, and annihilator-annihilator pairs. These solvent-based systems have routinely shown photon upconversion efficiencies matching the theoretical maximum of $50 \%$, whereas the absence of collisional interactions has made it challenging to achieve high efficiency upconversion in solid-state systems, where sensitizer and annihilator molecules are embedded, doped or used to form glassy polymer matrices. Examples of polymer systems exhibiting higher-efficiency TTA upconversion include structures that encourage triplet energy migration along annihilator chains, to a point where two migrating triplet energies 
interact and annihilate. Upconversion from this mechanism has been demonstrated in organized molecular systems, in which a low concentration of sensitizer molecules are integrated with densely accumulated annihilator molecules. Excitation of the sensitizer is followed by donor-to-acceptor TET, triplet energy migration (TEM) in the acceptor arrays, TTA between the excited acceptors, and finally, higher-energy UCL emission. The difference between conventional TTA and TEM-UCL is the favorable sensitizer-annihilator as well as annihilator-annihilator triplet energy transfer processes in the absence of free molecular diffusion, each of which are enabled by the preorganization of sensitizer-annihilator and annihilator-annihilator arrangements. Sensitizer-annihilator and annihilator-annihilator orbital overlaps are important for efficient TET and fast TEM. TET occurs via the Dexter energy transfer mechanism, and so it requires an overlap of wavefunctions between the donor and acceptor, thus possessing a steep exponential dependence on the distance. For high energy transfer quantum efficiency, the sensitizers and annihilators should be in contact. Additionally, annihilation distance between acceptor triplets is usually $<10 \AA$; the goal is thus to carefully design a molecular assembly structure so as to obtain fast triplet energy migration and high fluorescence quantum efficiency.

Unlike with lanthanide-based upconversion luminescence, the selection of sensitizers and annihilators used in TTA upconversion is broad and flexible. For sensitizers, the main requirements are: i) they should display efficient intersystem crossing; therefore, they often contain heavy atoms such as iodine, bromine, platinum, palladium, and ruthenium, that induce spin-orbit coupling and favor the formation of triplet states; ii) they should possess an energy gap similar to that of the annihilator for efficient energy transfer; and iii) they should not exhibit fast phosphorescence, which would lead to the loss of energy needed to transfer to annihilator's triplets. Typical sensitizers for TTA-based upconversion luminescence are porphyrin/phthalocyanine and polypyridine heavy- metal complexes, I or Br-substituted 
organic chromophores, and fullerene-chromophore dyads. The requirements for choosing good annihilators include i) a triplet state with energy suitable for the energy transfer process and a sufficiently long lifetime for the TTA process to occur, and ii) a high fluorescent quantum yield. Common annihilators include polycyclic aromatic hydrocarbons such as anthracene, pyrene, perylene, fluorene and their derivatives, and organic fluorophores such as BODIPY and diimide-based dyes.

TTA upconversion has been achieved in surface-anchored MOFs (SURMOFs) that are epitaxially grown to form layered heterojunction structures (73) [206]. In an A-B-A layered sandwich heterostructure, where B corresponds to the sensitizer that generates triplet excited states upon photon absorption, and A to the emitter that accepts these triplet states and emits a higher energy photon by TTA, triplet transfer across the A-B heterojunctions occurs via Dexter electron exchange (Figure 35). The B sensitizer layer is first excited with $532 \mathrm{~nm}$ light, to which A is transparent. Nearly all the photons absorbed by B at this stage generate triplet states within the layer, which then diffuse to the B-A heterojunction. Due to the short separation distance $(\sim 0.6 \mathrm{~nm})$ between the sensitizer and emitter layers, two electrons whose wavefunctions overlap are exchanged at the interface; with one being transferred from the emitter to the sensitizer and the other from the sensitizer to the emitter. This creates two triplets that annihilate, radiating a higher-energy blue photon. These SURMOF heterojunctions have quite high UCL thresholds $\left(\sim 25-120 \mathrm{mWcm}^{-2}\right)$ and low quantum yields $(1.8 \%)$. These are attributed to the trapping of triplets and intersystem crossing in the sensitizer B layer, and low quantum yields due to aggregate-like states, as well as a lossinducing red-shift in emission that was observed in time-resolved luminescence measurements in the emitter A layer. The authors were able to mitigate some of these unwanted effects by controlling the quality, thickness and ordering of the MOF heterostructures. For example, adding a sonication step in the heterostructure fabrication 
process leads to the formation of smoother SURMOF surfaces with lower defect concentrations, which was shown to reduce the upconversion threshold. The use of bilayer BA heterostructures also shows a significant decrease in upconversion threshold, down to 1 $\mathrm{mWcm}^{-2}$, as the thickness of the sensitizer B layer in increased. Even in the best case, however, the $\mathrm{B} \rightarrow \mathrm{A}$ triplet transfer efficiency is as low as $58 \%$, leaving plenty of room for design improvement.

A

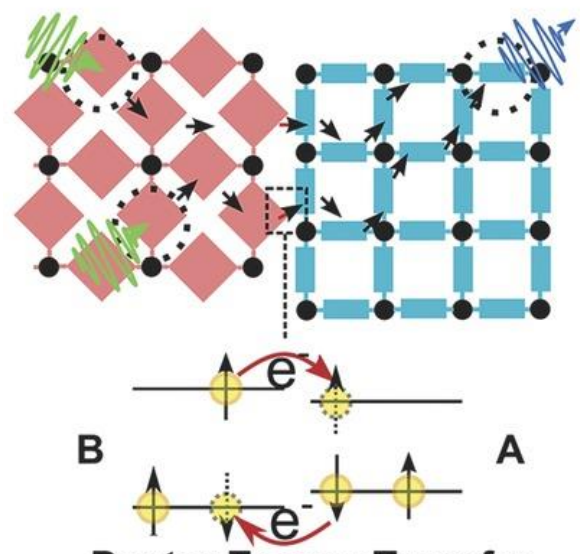

Dexter Energy Transfer

B

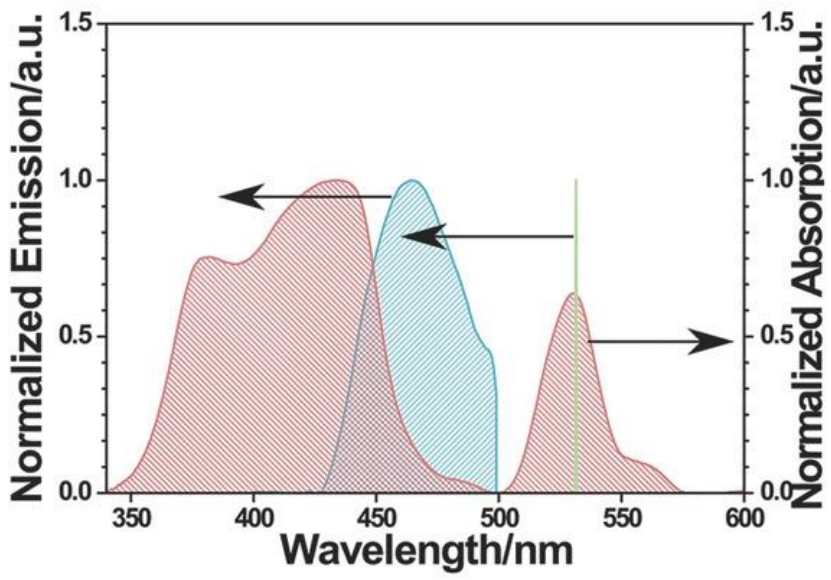

Figure 35. A) Schematic diagram of TTA UCL wherein two $532 \mathrm{~nm}$ photons absorbed by the sensitizer SURMOF create triplet states that upon reaching the interface can transfer to the emitter SURMOF by a Dexter two-electron exchange mechanism. When two triplets meet in the emitter layer they can annihilate and emit a single higher-energy photon. B) The absorption spectrum of sensitizer layer is shown in red and the wavelength of the $532 \mathrm{~nm}$ excitation laser is shown in green. The observed upconverted emission is shown in blue. The observation of upconverted emission provides direct optical evidence that the SURMOFSURMOF heterojunction is of sufficient quality to allow triplet, and therefore necessarily also electron, transfer between the SURMOF layers. Reproduced with permission from reference [206]. 


\section{Conclusions and Future Outlook}

In conclusion, due to the structural versatility and porosity of MOFs, they are considered ideal platforms for constructing materials that exhibit either luminescence or anti-Stokes shift luminescence, which can be utilized for applications in white-light emission, bioimaging, sensing, lasing, or photocatalysis. For most of these applications, improving the stability and efficiency of the MOF materials is the main target in the field. For example, water-stable MOFs are necessary to be synthesized in order to be used in upconversion photocatalysis. For luminescent MOFs that can be used for bioimaging, not only should they be stable in aqueous solution, but also they rather exhibit high quantum yield so that a very small amount of materials can be used, which prevents their toxicity towards biological cells and tissues. MOF-based sensors also require high chemical and thermal stability; for example, a MOFbased thermometer should retain its structural integrity over the range of temperatures that it senses. For NLO and UCL MOFs, more systematic studies are needed to understand the impact of ligand, metal ion, incorporated guest molecule, and framework topology - this area is still in its infancy. Nevertheless, the currently available results have unambiguously demonstrated that these MOFs can be very promising in many applications and we expect to see a lot more studies in the near future.

Another aspect that needs to be considered is the low productivity and processability of these MOFs for practical applications. For example, to replace the inorganic pigments in LEDs as a material for white-light emission, the MOF materials need to be synthesized on a much larger scale than in the conventional milligram-scale solvothermal/hydrothermal reactions. In addition, the powder form of luminescent MOFs is usually not preferable for sensing applications. We strongly believe that shaping luminescent MOF powders into membranes, 
films, pellets or beads [231] and associating these with simple devices will be the key for practical uses in real life.

\section{Acknowledgements}

TNN and KCS thank Swiss National Science Foundation (SNF) for funding under the Ambizione Energy Grant n.PZENP2_166888. The authors acknowledge the National Center of Competence in Research (NCCR), Materials' Revolution: Computational Design and Discovery of Novel Materials (MARVEL), of the Swiss National Science Foundation (SNSF) for funding - DD4.1 and DD4.5.

\section{References}

[1] O.M. Yaghi, H. Li, J. Am. Chem. Soc., 117 (1995) 10401-10402.

[2] O.K. Farha, I. Eryazici, N.C. Jeong, B.G. Hauser, C.E. Wilmer, A.A. Sarjeant, R.Q. Snurr, S.T. Nguyen, A.Ö. Yazaydın, J.T. Hupp, J. Am. Chem. Soc., 134 (2012) 15016-15021.

[3] H. Furukawa, Y.B. Go, N. Ko, Y.K. Park, F.J. Uribe-Romo, J. Kim, M. O’Keeffe, O.M. Yaghi, Inorg. Chem., 50 (2011) 9147-9152.

[4] H.-C. Zhou, J.R. Long, O.M. Yaghi, Chem. Rev., 112 (2012) 673-674.

[5] A. Schoedel, M. Li, D. Li, M. O’Keeffe, O.M. Yaghi, Chem. Rev., 116 (2016) 12466-12535.

[6] I. Burneo, K.C. Stylianou, S. Rodríguez-Hermida, J. Juanhuix, X. Fontrodona, I. Imaz, D. Maspoch, Cryst. Growth. Des., 15 (2015) 3182-3189.

[7] M. Witman, S. Ling, S. Anderson, L. Tong, K.C. Stylianou, B. Slater, B. Smit, M. Haranczyk, Chem. Sci., 7 (2016) 6263-6272.

[8] S. Rodríguez-Hermida, M.Y. Tsang, C. Vignatti, K.C. Stylianou, V. Guillerm, J. Pérez-Carvajal, F. Teixidor, C. Viñas, D. Choquesillo-Lazarte, C. Verdugo-Escamilla, I. Peral, J. Juanhuix, A. Verdaguer, I. Imaz, D. Maspoch, J.G. Planas, Angewandte Chemie International Edition, 55 (2016) 16049-16053.

[9] S. Ma, H.-C. Zhou, Chem. Commun., 46 (2010) 44-53.

[10] K. Sumida, D.L. Rogow, J.A. Mason, T.M. McDonald, E.D. Bloch, Z.R. Herm, T.-H. Bae, J.R. Long, Chem. Rev., 112 (2012) 724-781.

[11] M. Shah, M.C. McCarthy, S. Sachdeva, A.K. Lee, H.-K. Jeong, Ind. Eng. Chem. Res., 51 (2012) 2179-2199.

[12] Y.S. Lin, Curr. Opin. Chem. Eng., 8 (2015) 21-28.

[13] E. Adatoz, A.K. Avci, S. Keskin, Sep. Purif. Technol., 152 (2015) 207-237.

[14] Y. Lin, C. Kong, Q. Zhang, L. Chen, Adv. Energy Mater., 7 (2017) 1601296.

[15] M. Ranocchiari, J.A. van Bokhoven, Phys. Chem. Chem. Phys., 13 (2011) 6388-6396.

[16] J. Gascon, A. Corma, F. Kapteijn, F.X. Llabrés i Xamena, ACS Catal., 4 (2014) 361-378.

[17] A.H. Chughtai, N. Ahmad, H.A. Younus, A. Laypkov, F. Verpoort, Chem. Soc. Rev., 44 (2015) 6804-6849.

[18] Y. Zhao, Z. Song, X. Li, Q. Sun, N. Cheng, S. Lawes, X. Sun, Energy Storage Materials, 2 (2016) 3562.

[19] Y.-B. Huang, J. Liang, X.-S. Wang, R. Cao, Chem. Soc. Rev., 46 (2017) 126-157. 
[20] S. Kampouri, T.N. Nguyen, C.P. Ireland, B. Valizadeh, F.M. Ebrahim, G. Capano, D. Ongari, A. Mace, N. Guijarro, K. Sivula, A. Sienkiewicz, L. Forro, B. Smit, K.C. Stylianou, J. Mater. Chem. A, 6 (2018) 2476-2481.

[21] L.E. Kreno, K. Leong, O.K. Farha, M. Allendorf, R.P. Van Duyne, J.T. Hupp, Chem. Rev., 112 (2012) $1105-1125$.

[22] A. Gładysiak, T.N. Nguyen, J.A.R. Navarro, M.J. Rosseinsky, K.C. Stylianou, Chem. Eur. J., 23 (2017) 13602-13606.

[23] A. Chidambaram, K.C. Stylianou, Inorg. Chem. Front., 5 (2018) 979-998.

[24] L.R. Mingabudinova, V.V. Vinogradov, V.A. Milichko, E. Hey-Hawkins, A.V. Vinogradov, Chem. Soc. Rev., 45 (2016) 5408-5431.

[25] W. Zhang, R.G. Xiong, Chem. Rev., 112 (2012) 1163-1195.

[26] M. Kurmoo, Chem. Soc. Rev., 38 (2009) 1353-1379.

[27] A. Morozan, F. Jaouen, Energ. Environ. Sci., 5 (2012) 9269-9290.

[28] S. Horike, D. Umeyama, S. Kitagawa, Acc. Chem. Res., 46 (2013) 2376-2384.

[29] P. Ramaswamy, N.E. Wong, G.K. Shimizu, Chem. Soc. Rev., 43 (2014) 5913-5932.

[30] L. Sun, M.G. Campbell, M. Dincă, Angew. Chem. Int. Ed., 55 (2016) 3566-3579.

[31] P. Horcajada, T. Chalati, C. Serre, B. Gillet, C. Sebrie, T. Baati, J.F. Eubank, D. Heurtaux, P. Clayette, C. Kreuz, J.-S. Chang, Y.K. Hwang, V. Marsaud, P.-N. Bories, L. Cynober, S. Gil, G. Ferey, P. Couvreur, R. Gref, Nat. Mater., 9 (2010) 172-178.

[32] P. Horcajada, R. Gref, T. Baati, P.K. Allan, G. Maurin, P. Couvreur, G. Férey, R.E. Morris, C. Serre, Chem. Rev., 112 (2012) 1232-1268.

[33] M.-X. Wu, Y.-W. Yang, Adv. Mater., 29 (2017) 1606134.

[34] I. Mihad, S. Rana, A.H. Ghaleb, Curr. Med. Chem., 24 (2017) 193-214.

[35] M.D. Allendorf, C.A. Bauer, R.K. Bhakta, R.J.T. Houk, Chem. Soc. Rev., 38 (2009) 1330-1352.

[36] Y. Cui, Y. Yue, G. Qian, B. Chen, Chem. Rev., 112 (2012) 1126-1162.

[37] Z. Hu, B.J. Deibert, J. Li, Chem. Soc. Rev., 43 (2014) 5815-5840.

[38] D. Zhao, Y. Cui, Y. Yang, G. Qian, CrystEngComm, 18 (2016) 3746-3759.

[39] R. Medishetty, J.K. Zareba, D. Mayer, M. Samoc, R.A. Fischer, Chem. Soc. Rev., 46 (2017) $4976-$ 5004.

[40] M. Chalfie, Y. Tu, G. Euskirchen, W. Ward, D. Prasher, Science, 263 (1994) 802-805.

[41] S. Banerjee, C.D. Malliakas, J.I. Jang, J.B. Ketterson, M.G. Kanatzidis, J. Am. Chem. Soc., 130 (2008) 12270-12272.

[42] C. Dodeigne, L. Thunus, R. Lejeune, Talanta, 51 (2000) 415-439.

[43] U. Mitschke, P. Bauerle, J. Mater. Chem., 10 (2000) 1471-1507.

[44] D. Peng, B. Chen, F. Wang, ChemPlusChem, 80 (2015) 1209-1215.

[45] J. Van Houten, R.J. Watts, J. Am. Chem. Soc., 98 (1976) 4853-4858.

[46] W.W. Yu, L. Qu, W. Guo, X. Peng, Chem. Mater., 15 (2003) 2854-2860.

[47] C.M. McGraw, G. Khalil, J.B. Callis, J. Phys. Chem. C, 112 (2008) 8079-8084.

[48] J.R. Lakowicz, Long-Lifetime Metal-Ligand Complexes, in: Principles of Fluorescence Spectroscopy, Springer US, Boston, MA, 1999, pp. 573-594.

[49] V.W.-W. Yam, K.M.-C. Wong, Chem. Commun., 47 (2011) 11579-11592.

[50] K. Suzuki, A. Kobayashi, S. Kaneko, K. Takehira, T. Yoshihara, H. Ishida, Y. Shiina, S. Oishi, S. Tobita, Phys. Chem. Chem. Phys., 11 (2009) 9850-9860.

[51] J.L. Banal, B. Zhang, D.J. Jones, K.P. Ghiggino, W.W.H. Wong, Acc. Chem. Res., 1 (2016) 49-57.

[52] M.S.T. Gonçalves, Chem. Rev., 109 (2009) 190-212.

[53] N. Panchuk-Voloshina, R.P. Haugland, J. Bishop-Stewart, M.K. Bhalgat, P.J. Millard, F. Mao, W.-Y. Leung, R.P. Haugland, J. Histochem. Cytochem., 47 (1999) 1179-1188.

[54] D. Hu, Y. Song, L. Wang, J. Nanopart. Res., 17 (2015) 310.

[55] W. Pham, Z. Medarova, A. Moore, Bioconjugate Chem., 16 (2005) 735-740.

[56] Z. Zhang, S. Achilefu, Org. Lett., 6 (2004) 2067-2070.

[57] A.W. Adamson, J.N. Demas, J. Am. Chem. Soc., 93 (1971) 1800-1801. 
[58] J.P. Sauvage, J.P. Collin, J.C. Chambron, S. Guillerez, C. Coudret, V. Balzani, F. Barigelletti, L. De Cola, L. Flamigni, Chem. Rev., 94 (1994) 993-1019.

[59] M. Wrighton, D.L. Morse, J. Am. Chem. Soc., 96 (1974) 998-1003.

[60] A.J. Lees, Chem. Rev., 87 (1987) 711-743.

[61] J.V. Caspar, T.J. Meyer, J. Phys. Chem., 87 (1983) 952-957.

[62] R.E. DeSimone, R.S. Drago, Inorg. Chem., 8 (1969) 2517-2519.

[63] I.M. Dixon, J.-P. Collin, J.-P. Sauvage, L. Flamigni, S. Encinas, F. Barigelletti, Chem. Soc. Rev., 29 (2000) 385-391.

[64] J.A.G. Williams, Chem. Soc. Rev., 38 (2009) 1783-1801.

[65] K.K.-W. Lo, S.P.-Y. Li, K.Y. Zhang, New J. Chem., 35 (2011) 265-287.

[66] E.I. Mayo, K. Kilsa, T. Tirrell, P.I. Djurovich, A. Tamayo, M.E. Thompson, N.S. Lewis, H.B. Gray, Photochem. Photobiol. Sci., 5 (2006) 871-873.

[67] P. Didier, I. Ortmans, A. Kirsch-De Mesmaeker, R.J. Watts, Inorg. Chem., 32 (1993) 5239-5245.

[68] S.J. Lippard, Acc. Chem. Res., 11 (1978) 211-217.

[69] V.W.-W. Yam, V.K.-M. Au, S.Y.-L. Leung, Chem. Rev., 115 (2015) 7589-7728.

[70] V.W.-W. Yam, R.P.-L. Tang, K.M.-C. Wong, K.-K. Cheung, Organometallics, 20 (2001) 4476-4482.

[71] H. Schmidbaur, Chem. Soc. Rev., 24 (1995) 391-400.

[72] V.W.-W. Yam, E.C.-C. Cheng, K.-K. Cheung, Angew. Chem. Int. Ed., 38 (1999) 197-199.

[73] T.K.-M. Lee, N. Zhu, V.W.-W. Yam, J. Am. Chem. Soc., 132 (2010) 17646-17648.

[74] S. Lebedkin, T. Langetepe, P. Sevillano, D. Fenske, M.M. Kappes, J. Phys. Chem. B, 106 (2002) 9019-9026.

[75] M.H. Keefe, K.D. Benkstein, J.T. Hupp, Coord. Chem. Rev., 205 (2000) 201-228.

[76] J.N. Demas, B.A. DeGraff, Coord. Chem. Rev., 211 (2001) 317-351.

[77] D.-L. Ma, V.P.-Y. Ma, D.S.-H. Chan, K.-H. Leung, H.-Z. He, C.-H. Leung, Coord. Chem. Rev., 256 (2012) 3087-3113.

[78] K.K.-W. Lo, M.-W. Louie, K.Y. Zhang, Coord. Chem. Rev., 254 (2010) 2603-2622.

[79] D. Lloyd, M.P. Coogan, S.J.A. Pope, Novel Metal-Based Luminophores for Biological Imaging, in:

C.D. Geddes (Ed.) Reviews in Fluorescence 2010, Springer New York, New York, NY, 2012, pp. 15-44.

[80] Y. Sun, N.C. Giebink, H. Kanno, B. Ma, M.E. Thompson, S.R. Forrest, Nature, 440 (2006) 908-912.

[81] A. Santoro, A.M. Prokhorov, V.N. Kozhevnikov, A.C. Whitwood, B. Donnio, J.A.G. Williams, D.W. Bruce, J. Am. Chem. Soc., 133 (2011) 5248-5251.

[82] B.W. D’Andrade, J. Brooks, V. Adamovich, M.E. Thompson, S.R. Forrest, Adv. Mater., 14 (2002) 1032-1036.

[83] S. Welter, K. Brunner, J.W. Hofstraat, L. De Cola, Nature, 421 (2003) 54-57.

[84] B. Carlson, G.D. Phelan, W. Kaminsky, L. Dalton, X. Jiang, S. Liu, A.K.Y. Jen, J. Am. Chem. Soc., 124 (2002) 14162-14172.

[85] B. O'Regan, M. Gratzel, Nature, 353 (1991) 737-740.

[86] M. Grätzel, Acc. Chem. Res., 42 (2009) 1788-1798.

[87] G. Sauvé, M.E. Cass, G. Coia, S.J. Doig, I. Lauermann, K.E. Pomykal, N.S. Lewis, J. Phys. Chem. B, 104 (2000) 6821-6836.

[88] T. Bessho, E.C. Constable, M. Graetzel, A. Hernandez Redondo, C.E. Housecroft, W. Kylberg, M.K. Nazeeruddin, M. Neuburger, S. Schaffner, Chem. Commun., (2008) 3717-3719.

[89] P. Du, J. Schneider, F. Li, W. Zhao, U. Patel, F.N. Castellano, R. Eisenberg, J. Am. Chem. Soc., 130 (2008) 5056-5058.

[90] A.J. Morris, G.J. Meyer, E. Fujita, Acc. Chem. Res., 42 (2009) 1983-1994.

[91] A.J. Esswein, D.G. Nocera, Chem. Rev., 107 (2007) 4022-4047.

[92] R. Rossetti, L. Brus, J. Phys. Chem., 86 (1982) 4470-4472.

[93] K.D. Wegner, N. Hildebrandt, Chem. Soc. Rev., 44 (2015) 4792-4834.

[94] H. Weller, Angew. Chem. Int. Ed., 32 (1993) 41-53.

[95] W.R. Algar, K. Susumu, J.B. Delehanty, I.L. Medintz, Anal. Chem., 83 (2011) 8826-8837.

[96] R. Tang, J. Xue, B. Xu, D. Shen, G.P. Sudlow, S. Achilefu, ACS Nano, 9 (2015) 220-230. 
[97] L. Martínez Maestro, C. Jacinto, U. Rocha, M. Carmen Iglesias-de la Cruz, F. Sanz-Rodriguez, A. Juarranz, J. García Solé, D. Jaque, J. Appl. Phys., 111 (2012) 023513.

[98] V. Biju, T. Itoh, M. Ishikawa, Chem. Soc. Rev., 39 (2010) 3031-3056.

[99] P. Alivisatos, Nat. Biotech., 22 (2004) 47-52.

[100] W.C.W. Chan, S. Nie, Science, 281 (1998) 2016-2018.

[101] X. Gao, L. Yang, J.A. Petros, F.F. Marshall, J.W. Simons, S. Nie, Curr. Opin. Biotech., 16 (2005) 63-72.

[102] L. Manna, E.C. Scher, L.-S. Li, A.P. Alivisatos, J. Am. Chem. Soc., 124 (2002) 7136-7145.

[103] M.A. Hines, P. Guyot-Sionnest, J. Phys. Chem., 100 (1996) 468-471.

[104] D. Gerion, F. Pinaud, S.C. Williams, W.J. Parak, D. Zanchet, S. Weiss, A.P. Alivisatos, J. Phys. Chem. B, 105 (2001) 8861-8871.

[105] B. Dubertret, P. Skourides, D.J. Norris, V. Noireaux, A.H. Brivanlou, A. Libchaber, Science, 298 (2002) 1759.

[106] S. Kim, M.G. Bawendi, J. Am. Chem. Soc., 125 (2003) 14652-14653.

[107] J.H.V. Vleck, J. Phys. Chem., 41 (1937) 67-80.

[108] J.-C.G. Bünzli, S. Comby, A.-S. Chauvin, C.D.B. Vandevyver, J. Rare Earth, 25 (2007) 257-274.

[109] P.R. Selvin, IEEE J. Sel. Top. Quant., 2 (1996) 1077-1087.

[110] L. Armelao, S. Quici, F. Barigelletti, G. Accorsi, G. Bottaro, M. Cavazzini, E. Tondello, Coord. Chem. Rev., 254 (2010) 487-505.

[111] J.-C.G. Bunzli, S.V. Eliseeva, Chem. Sci., 4 (2013) 1939-1949.

[112] S.V. Eliseeva, J.-C.G. Bunzli, Chem. Soc. Rev., 39 (2010) 189-227.

[113] T.N. Nguyen, G. Capano, A. Gladysiak, F.M. Ebrahim, S.V. Eliseeva, A. Chidambaram, B. Valizadeh, S. Petoud, B. Smit, K.C. Stylianou, Chem. Commun., 54 (2018) 6816-6819.

[114] L. Song, J. Hu, J. Wang, X. Liu, Z. Zhen, Photochem. Photobiol. Sci., 7 (2008) 689-693.

[115] P.B. Glover, A.P. Bassett, P. Nockemann, B.M. Kariuki, R. Van Deun, Z. Pikramenou, Chem. Eur. J., 13 (2007) 6308-6320.

[116] J.-Y. Hu, Y. Ning, Y.-S. Meng, J. Zhang, Z.-Y. Wu, S. Gao, J.-L. Zhang, Chem. Sci., 8 (2017) 27022709.

[117] C.Y. Chow, S.V. Eliseeva, E.R. Trivedi, T.N. Nguyen, J.W. Kampf, S. Petoud, V.L. Pecoraro, J. Am. Chem. Soc., 138 (2016) 5100-5109.

[118] T.N. Nguyen, C.Y. Chow, S.V. Eliseeva, E.R. Trivedi, J.W. Kampf, I. Martinić, S. Petoud, V.L. Pecoraro, Chem. Eur. J., 24 1031-1035.

[119] I. Martinić, S.V. Eliseeva, T.N. Nguyen, V.L. Pecoraro, S. Petoud, J. Am. Chem. Soc., 139 (2017) 8388-8391.

[120] I. Martinic, S.V. Eliseeva, T.N. Nguyen, F. Foucher, D. Gosset, F. Westall, V.L. Pecoraro, S. Petoud, Chem. Sci., 8 (2017) 6042-6050.

[121] J. Rocha, C.D.S. Brites, L.D. Carlos, Chem. Eur. J., 22 (2016) 14782-14795.

[122] R.-B. Lin, S.-Y. Liu, J.-W. Ye, X.-Y. Li, J.-P. Zhang, Adv. Sci., 3 (2016) 1500434.

[123] T. Cheng, J. Hu, C. Zhou, Y. Wang, M. Zhang, Sci. China Chem., 59 (2016) 929-947.

[124] Y. Cui, B. Chen, G. Qian, Coord. Chem. Rev., 273-274 (2014) 76-86.

[125] Y. Zhang, S. Yuan, G. Day, X. Wang, X. Yang, H.-C. Zhou, Coord. Chem. Rev., 354 (2018) 28-45.

[126] W.P. Lustig, S. Mukherjee, N.D. Rudd, A.V. Desai, J. Li, S.K. Ghosh, Chem. Soc. Rev., 46 (2017) 3242-3285.

[127] L. Ma, X. Feng, S. Wang, B. Wang, Mater. Chem. Front., 1 (2017) 2474-2486.

[128] R. Li, S.-H. Wang, Z.-F. Liu, X.-X. Chen, Y. Xiao, F.-K. Zheng, G.-C. Guo, Cryst. Growth. Des., 16 (2016) 3969-3975.

[129] J. He, M. Zeller, A.D. Hunter, Z. Xu, J. Am. Chem. Soc., 134 (2012) 1553-1559.

[130] M.-S. Wang, S.-P. Guo, Y. Li, L.-Z. Cai, J.-P. Zou, G. Xu, W.-W. Zhou, F.-K. Zheng, G.-C. Guo, J. Am. Chem. Soc., 131 (2009) 13572-13573.

[131] D.F. Sava, L.E.S. Rohwer, M.A. Rodriguez, T.M. Nenoff, J. Am. Chem. Soc., 134 (2012) 39833986. 
[132] J. Park, M. Oh, CrystEngComm, 18 (2016) 8372-8376.

[133] Y. Gai, Q. Guo, K. Xiong, F. Jiang, C. Li, X. Li, Y. Chen, C. Zhu, Q. Huang, R. Yao, M. Hong, Cryst. Growth. Des., 17 (2017) 940-944.

[134] T. Song, G. Zhang, Y. Cui, Y. Yang, G. Qian, CrystEngComm, 18 (2016) 8366-8371.

[135] Z. Wang, Z. Wang, B. Lin, X. Hu, Y. Wei, C. Zhang, B. An, C. Wang, W. Lin, ACS Appl. Mater. Interfaces, 9 (2017) 35253-35259.

[136] Y. Cui, T. Song, J. Yu, Y. Yang, Z. Wang, G. Qian, Adv. Funct. Mater, 25 (2015) 4796-4802.

[137] Y. Wen, T. Sheng, X. Zhu, C. Zhuo, S. Su, H. Li, S. Hu, Q.-L. Zhu, X. Wu, Adv. Mater., 29 (2017) 1700778-n/a.

[138] C.-Y. Sun, X.-L. Wang, X. Zhang, C. Qin, P. Li, Z.-M. Su, D.-X. Zhu, G.-G. Shan, K.-Z. Shao, H. Wu, J. Li, Nat. Commun., 4 (2013) 2717.

[139] W. Xie, W.-W. He, D.-Y. Du, S.-L. Li, J.-S. Qin, Z.-M. Su, C.-Y. Sun, Y.-Q. Lan, Chem. Commun., 52 (2016) 3288-3291.

[140] T. Kundu, S. Mitra, D. Díaz Díaz, R. Banerjee, ChemPlusChem, 81 (2016) 728-732.

[141] D. Liu, R.C. Huxford, W. Lin, Angew. Chem. Int. Ed., 50 (2011) 3696-3700.

[142] A. Foucault-Collet, K.A. Gogick, K.A. White, S. Villette, A. Pallier, G. Collet, C. Kieda, T. Li, S.J. Geib, N.L. Rosi, S. Petoud, Proc. Natl. Acad. Sci. USA, 110 (2013) 17199-17204.

[143] K.M.L. Taylor-Pashow, J.D. Rocca, Z. Xie, S. Tran, W. Lin, J. Am. Chem. Soc., 131 (2009) 1426114263.

[144] R. Chen, J. Zhang, J. Chelora, Y. Xiong, S.V. Kershaw, K.F. Li, P.-K. Lo, K.W. Cheah, A.L. Rogach, J.A. Zapien, C.-S. Lee, ACS Appl. Mater. Interfaces, 9 (2017) 5699-5708.

[145] H. Zhang, C. Lin, T. Sheng, S. Hu, C. Zhuo, R. Fu, Y. Wen, H. Li, S. Su, X. Wu, Chem. Eur. J., 22 (2016) 4460-4468.

[146] Y. Yang, L. Chen, F. Jiang, M. Yu, X. Wan, B. Zhang, M. Hong, J. Mater. Chem. C, 5 (2017) 19811989.

[147] X. Lian, D. Zhao, Y. Cui, Y. Yang, G. Qian, Chem. Commun., 51 (2015) 17676-17679.

[148] T. Xia, Y. Cui, Y. Yang, G. Qian, J. Mater. Chem. C, 5 (2017) 5044-5047.

[149] Y. Cui, R. Song, J. Yu, M. Liu, Z. Wang, C. Wu, Y. Yang, Z. Wang, B. Chen, G. Qian, Adv. Mater., 27 (2015) 1420-1425.

[150] J. Aguilera-Sigalat, D. Bradshaw, Chem. Commun., 50 (2014) 4711-4713.

[151] X.-Y. Xu, B. Yan, Dalton Trans., 45 (2016) 7078-7084.

[152] H.-Y. Li, Y.-L. Wei, X.-Y. Dong, S.-Q. Zang, T.C.W. Mak, Chem. Mater., 27 (2015) 1327-1331.

[153] X. Zhang, K. Jiang, H. He, D. Yue, D. Zhao, Y. Cui, Y. Yang, G. Qian, Sensor Actuat B-Chem., 254 (2018) 1069-1077.

[154] J. Wang, Y. Li, M. Jiang, Y. Liu, L. Zhang, P. Wu, Chem. Eur. J., 22 (2016) 13023-13027.

[155] T. Xia, F. Zhu, K. Jiang, Y. Cui, Y. Yu, G. Qian, Dalton Trans., 46 (2017) 7549-7555.

[156] S. Bhattacharyya, A. Chakraborty, K. Jayaramulu, A. Hazra, T.K. Maji, Chem. Commun., 50 (2014) 13567-13570.

[157] M. Carboni, Z. Lin, C.W. Abney, T. Zhang, W. Lin, Chem. Eur. J., 20 (2014) 14965-14970.

[158] X.-L. Zhao, D. Tian, Q. Gao, H.-W. Sun, J. Xu, X.-H. Bu, Dalton Trans., 45 (2016) 1040-1046.

[159] W. Yan, C. Zhang, S. Chen, L. Han, H. Zheng, ACS Appl. Mater. Interfaces, 9 (2017) 1629-1634.

[160] Y. Ma, G. Xu, F. Wei, Y. Cen, Y. Ma, Y. Song, X. Xu, M. Shi, S. Muhammad, Q. Hu, J. Mater. Chem. C, 5 (2017) 8566-8571.

[161] Y. Lu, B. Yan, J.-L. Liu, Chem. Commun., 50 (2014) 9969-9972.

[162] N.D. Rudd, H. Wang, E.M.A. Fuentes-Fernandez, S.J. Teat, F. Chen, G. Hall, Y.J. Chabal, J. Li, ACS Appl. Mater. Interfaces, 8 (2016) 30294-30303.

[163] K.S. Lim, S.Y. Jeong, D.W. Kang, J.H. Song, H. Jo, W.R. Lee, W.J. Phang, D. Moon, C.S. Hong, Chem. Eur. J., 23 (2017) 4803-4809.

[164] W.-M. Chen, X.-L. Meng, G.-L. Zhuang, Z. Wang, M. Kurmoo, Q.-Q. Zhao, X.-P. Wang, B. Shan, C.-H. Tung, D. Sun, J. Mater. Chem. A, 5 (2017) 13079-13085.

[165] J.-N. Hao, B. Yan, J. Mater. Chem. A, 2 (2014) 18018-18025. 
[166] F.-Y. Yi, J.-P. Li, D. Wu, Z.-M. Sun, Chem. Eur. J., 21 (2015) 11475-11482.

[167] J.-M. Zhou, W. Shi, H.-M. Li, H. Li, P. Cheng, J. Phys. Chem. C, 118 (2014) 416-426.

[168] P.-F. Shi, H.-C. Hu, Z.-Y. Zhang, G. Xiong, B. Zhao, Chem. Commun., 51 (2015) 3985-3988.

[169] Z.-R. Yang, M.-M. Wang, X.-S. Wang, X.-B. Yin, Anal. Chem., 89 (2017) 1930-1936.

[170] J. Roales, F. Moscoso, F. Gámez, T. Lopes-Costa, A. Sousaraei, S. Casado, J. Castro-Smirnov, J. Cabanillas-Gonzalez, J. Almeida, C. Queirós, L. Cunha-Silva, A. Silva, J. Pedrosa, Materials, 10 (2017) 992.

[171] X.-Y. Xu, B. Yan, J. Mater. Chem. C, 4 (2016) 8514-8521.

[172] J. Zhang, T. Xia, D. Zhao, Y. Cui, Y. Yang, G. Qian, Sensor Actuat B-Chem., 260 (2018) 63-69.

[173] R.-W. Huang, Y.-S. Wei, X.-Y. Dong, X.-H. Wu, C.-X. Du, S.-Q. Zang, T.C.W. Mak, Nat. Chem., 9 (2017) 689.

[174] J. Li, X. Luo, Y. Zhou, L. Zhang, Q. Huo, Y. Liu, Cryst. Growth. Des., 18 (2018) 1857-1863.

[175] S.-L. Hou, J. Dong, X.-L. Jiang, Z.-H. Jiao, C.-M. Wang, B. Zhao, Anal. Chem., (2018).

[176] D. Yue, D. Zhao, J. Zhang, L. Zhang, K. Jiang, X. Zhang, Y. Cui, Y. Yang, B. Chen, G. Qian, Chem. Commun., 53 (2017) 11221-11224.

[177] Y. Gao, G. Yu, K. Liu, B. Wang, Sensor Actuat B-Chem., 257 (2018) 931-935.

[178] N. Armaroli, V. Balzani, Energ. Environ. Sci., 4 (2011) 3193-3222.

[179] P. Von Dollen, S. Pimputkar, J.S. Speck, Angew. Chem. Int. Ed., 53 (2014) 13978-13980.

[180] S.S. Mondal, K. Behrens, P.R. Matthes, F. Schonfeld, J. Nitsch, A. Steffen, P.-A. Primus, M.U. Kumke, K. Muller-Buschbaum, H.-J. Holdt, J. Mater. Chem. C, 3 (2015) 4623-4631.

[181] F. Yang, L. Wei, T.S. J., D. Gangotri, S. Zeqing, A. Litao, Y. Dechao, W. Lu, O.C.D. M., L. Jing, Adv. Funct. Mater., 27 (2017) 1603444.

[182] D. Liu, K. Lu, C. Poon, W. Lin, Inorg. Chem., 53 (2014) 1916-1924.

[183] S.-D. Li, Y.-C. Chen, M.J. Hackett, L. Huang, Mol. Ther., 16 (2008) 163-169.

[184] K.P. Carter, A.M. Young, A.E. Palmer, Chem. Rev., 114 (2014) 4564-4601.

[185] L. Tomljenovic, J. Alzheimers Dis., 23 (2011) 567-598.

[186] U.S.EPA, in: Final/Technical Report, Washinton, DC, 1980.

[187] F.-Y. Yi, D. Chen, M.-K. Wu, L. Han, H.-L. Jiang, ChemPlusChem, 81 (2016) 675-690.

[188] Z.-Q. Yao, G.-Y. Li, J. Xu, T.-L. Hu, X.-H. Bu, Chem. Eur. J., 24 3192-3198.

[189] http://www.who.int/water sanitation health/dwq/chemicals/fluoride.pdf.

[190] L. Zhang, Z. Kang, X. Xin, D. Sun, CrystEngComm, 18 (2016) 193-206.

[191] Z. Jingrong, T. Gan, Z. Lijuan, S. Xueer, B. Xiu-wu, Adv. Healthcare Mater., 7 (2018) 1800022.

[192] H. Wang, W.P. Lustig, J. Li, Chem. Soc. Rev., (2018).

[193] A. Lan, K. Li, H. Wu, D.H. Olson, T.J. Emge, W. Ki, M. Hong, J. Li, Angew. Chem. Int. Ed., 48 (2009) 2334-2338.

[194] O.R. Evans, R.G. Xiong, Z.Y. Wang, G.K. Wong, W.B. Lin, Angew. Chem. Int. Ed., 38 (1999) 536538.

[195] L.L. Liang, S.B. Ren, J. Zhang, Y.Z. Li, H.B. Du, X.Z. You, Dalton Trans., 39 (2010) 7723-7726.

[196] Y.T. Wang, H.H. Fan, H.Z. Wang, X.M. Chen, Inorg. Chem., 44 (2005) 4148-4150.

[197] J. Yu, Y. Cui, C. Wu, Y. Yang, Z. Wang, M. O'Keeffe, B. Chen, G. Qian, Angew. Chem. Int. Ed., 51 (2012) 10542-10545.

[198] Y. Liu, G. Li, X. Li, Y. Cui, Angew. Chem. Int. Ed., 46 (2007) 6301-6304.

[199] J. Yu, Y. Cui, C.-D. Wu, Y. Yang, B. Chen, G. Qian, J. Am. Chem. Soc., 137 (2015) 4026-4029.

[200] H.S. Quah, W. Chen, M.K. Schreyer, H. Yang, M.W. Wong, W. Ji, J.J. Vittal, Nat. Commun., 6 (2015) 7954.

[201] R. Medishetty, L. Nemec, V. Nalla, S. Henke, M. Samoc, K. Reuter, R.A. Fischer, Angew. Chem. Int. Ed., 56 (2017) 14743-14748.

[202] X. Zhang, B. Li, H. Ma, L. Zhang, H. Zhao, ACS Appl Mater Interfaces, 8 (2016) 17389-17394.

[203] C. Liu, B. Yan, New J. Chem., 39 (2015) 1125-1131.

[204] Y. Li, J. Tang, L. He, Y. Liu, Y. Liu, C. Chen, Z. Tang, Adv. Mater., 27 (2015) 4075-4080. 
[205] M.H. Li, Z.J. Zheng, Y.Q. Zheng, C. Cui, C.X. Li, Z.Q. Li, ACS Appl. Mater. Interfaces, 9 (2017) 2899-2905.

[206] M. Oldenburg, A. Turshatov, D. Busko, S. Wollgarten, M. Adams, N. Baroni, A. Welle, E. Redel, C. Woll, B.S. Richards, I.A. Howard, Adv. Mater., 28 (2016) 8477-8482.

[207] R.W. Boyd, Nonlinear optics, Academic Press, Boston etc., 1992.

[208] C. Wang, T. Zhang, W. Lin, Chem. Rev., 112 (2012) 1084-1104.

[209] F. Zernike, J.E. Midwinter, Applied nonlinear optics, Wiley, 1973.

[210] The efficiency of novel SHG materials is often compared with that of known standards such as urea, and the crystals mentioned above. However, it is difficult to accurately assess the absolute performance of a new material this way; for example, the SHG efficiency of urea is roughly six times greater than that of KDP at $\lambda_{\mathrm{ex}}=1064 \mathrm{~nm}$, which in turn is ten times higher than that of $\alpha$-quartz. For the sake of clarity, we compare the performance of the reported SHG-active MOF structures with that of KDP where relevant.

[211] Y.Q. Zheng, J. Zhang, J.Y. Liu, CrystEngComm, 12 (2010) 2740-2748.

[212] K. Biradha, C. Seward, M.J. Zaworotko, Angew. Chem. Int. Ed., 38 (1999) 492-495.

[213] T. Ezuhara, K. Endo, Y. Aoyama, J. Am. Chem. Soc., 121 (1999) 3279-3283.

[214] O.R. Evans, W.B. Lin, Acc. Chem. Res., 35 (2002) 511-522.

[215] S.R. Batten, S.M. Neville, D.R. Turner, Coordination polymers design, analysis and application, Royal Society of Chemistry, Cambridge, 2009.

[216] M. Liu, H.S. Quah, S. Wen, Z. Yu, J.J. Vittal, W. Ji, Chem. Mater., 28 (2016) 3385-3390.

[217] R.W. Boyd, A.L. Gaeta, Laser Optics of Condensed Matter, Vol 2, (1991) 99-105.

[218] F. Auzel, Luminescense : Phenomena, Materials, and Devices, (1992) 33-41.

[219] J. Zhou, Q. Liu, W. Feng, Y. Sun, F. Li, Chem. Rev., 115 (2015) 395-465.

[220] L.Y. Ang, M.E. Lim, L.C. Ong, Y. Zhang, Nanomedicine-Uk, 6 (2011) 1273-1288.

[221] P. Ramasamy, P. Manivasakan, J. Kim, Rsc. Adv., 4 (2014) 34873-34895.

[222] X.Y. Huang, S.Y. Han, W. Huang, X.G. Liu, Chem. Soc. Rev., 42 (2013) 173-201.

[223] Y.Y. Cheng, B. Fuckel, R.W. MacQueen, T. Khoury, R.G.C.R. Clady, T.F. Schulze, N.J. EkinsDaukes, M.J. Crossley, B. Stannowski, K. Lips, T.W. Schmidt, Energ. Environ. Sci., 5 (2012) 6953-6959.

[224] W.G.J.H.M. van Sark, J. de Wild, J.K. Rath, A. Meijerink, R.E.I. Schropp, Nanoscale Res. Lett., 8 (2013).

[225] F. Auzel, Chem. Rev., 104 (2004) 139-173.

[226] W.P. Qin, Z.Y. Liu, C.N. Sin, C.F. Wu, G.S. Qin, Z. Chen, K.Z. Zheng, Light-Sci. Appl., 3 (2014).

[227] F. Varsanyi, G.H. Dieke, Phys. Rev. Lett., 7 (1961) 442-\&.

[228] D.L. Dexter, Phys. Rev., 126 (1962) 1962-\&.

[229] G. Lu, S.Z. Li, Z. Guo, O.K. Farha, B.G. Hauser, X.Y. Qi, Y. Wang, X. Wang, S.Y. Han, X.G. Liu, J.S. DuChene, H. Zhang, Q.C. Zhang, X.D. Chen, J. Ma, S.C.J. Loo, W.D. Wei, Y.H. Yang, J.T. Hupp, F.W. Huo, Nat. Chem., 4 (2012) 310-316.

[230] K.R. Deng, Z.Y. Hou, X.J. Li, C.X. Li, Y.X. Zhang, X.R. Deng, Z.Y. Cheng, J. Lin, Sci. Rep., 5 (2015).

[231] B. Valizadeh, T.N. Nguyen, K.C. Stylianou, Polyhedron, 145 (2018) 1-15. 